\title{
Air-Stable PinP(O)H as Preligand for Palladium-Catalyzed Kumada Couplings of Unactivated Tosylates
}

\author{
Lutz Ackermann,* and Andreas Althammer
}

General remarks

Catalytic reactions were carried out on a 1 mmol scale under a $\mathrm{N}_{2}$ atmosphere using pre-dried glassware. Chemicals were obtained from commercial sources, and were used without further purification. Aryltosylates were prepared as described in the literature. ${ }^{1}$ Dioxane was freshly distilled from sodium/benzophenone under $\mathrm{N}_{2}$. Yields refer to isolated compounds, estimated to be $>95 \%$ pure as determined by ${ }^{1} \mathrm{H}-\mathrm{NMR}$ and GC. Flash chromatography: Macherey-Nagel silica gel 60 (70-230 mesh). NMR: Spectra were recorded on a Bruker ARX 300 or on a Varian VXR 400 instrument in the solvent indicated; chemical shifts $(\delta)$ are given in ppm. 
Representative procedure for palladium-catalyzed Kumada crosscoupling reactions of aryl tosylates (Table 1, entry 11):

A solution of $\left[\mathrm{Pd}(\mathrm{dba})_{2}\right](2.9 \mathrm{mg}, 0.005 \mathrm{mmol}, 0.5 \mathrm{~mol} \%)$ and PinP (O) H (12) (1.6 mg, $0.010 \mathrm{mmol}, 1.0 \mathrm{~mol} \%)$ in dry dioxane $\left(4.0 \mathrm{~mL}\right.$ ) was stirred under $\mathrm{N}_{2}$ for $5 \mathrm{~min}$ at ambient temperature, and then treated with $\mathbf{2 a}(3.0 \mathrm{~mL}, 0.5 \mathrm{M}$ in THF, $1.50 \mathrm{mmol})$. The resulting mixture was stirred for 5 min at ambient temperature. Thereafter, $\mathbf{1} \mathbf{a}$ was added, and the resulting suspension was stirred at $80{ }^{\circ} \mathrm{C}$ for $22 \mathrm{~h}$. At ambient temperature, aqueous $\mathrm{HCl}(2.0 \mathrm{~mL}, 2.0 \mathrm{~N}), \mathrm{Et}_{2} \mathrm{O}(50 \mathrm{~mL})$, and $\mathrm{H}_{2} \mathrm{O}$ (30 $\mathrm{mL})$ were added. The separated aqueous phase was extracted with $\mathrm{Et}_{2} \mathrm{O}(2 \times 50 \mathrm{~mL})$. The combined organic layers were dried over $\mathrm{MgSO}_{4}$ and concentrated in vacuo. The remaining residue was purified by column chromatography on silica gel ( $n$-pentane/Et ${ }_{2} \mathrm{O}$ $=200 / 1$ ) to yield $3 \mathbf{a}(171 \mathrm{mg}, 93$ o) as a colorless solid (mp: 85.1-85.4 $\left.{ }^{\circ} \mathrm{C}\right)$. The spectral data were in accordance with those reported in the literature. ${ }^{2}$

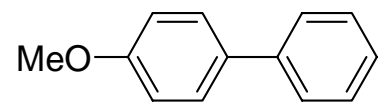

4-Methoxybiphenyl (Table 2, entry 1):

The representative procedure was followed, using [Pd(dba) 2 ] (14.4 mg, $0.025 \mathrm{mmol}, 2.5 \mathrm{~mol} \%), 12$ (8.2 mg, $0.050 \mathrm{mmol}$, 5.0 molo), phenylmagnesiumchloride $(1.5 \mathrm{M}$ in $\mathrm{THF}, 1.0 \mathrm{~mL}$, $1.50 \mathrm{mmol})$ and 4-methoxyphenyltosylate (278 $\mathrm{mg}, 1.00 \mathrm{mmol})$. After $22 \mathrm{~h}$, purification by chromatography (n-pentane/Et ${ }_{2} \mathrm{O}=$ 
$200 / 1$ ) yielded 4-methoxybiphenyl (172 mg, $0.93 \mathrm{mmol}, 93$ 을 as a colourless solid (mp: $85.3-85.7^{\circ} \mathrm{C}$ ). The spectral data were in accordance with those reported in the literature. ${ }^{2}$

${ }^{1} \mathrm{H}-\mathrm{NMR} \quad\left(300 \mathrm{MHz}, \mathrm{CDCl}_{3}\right): \delta=7.59-7.53(\mathrm{~m}, 4 \mathrm{H}), 7.43(t, J=$ $7.3 \mathrm{~Hz}, 2 \mathrm{H}), 7.33$ (tt, $J=7.3 \mathrm{~Hz}, 1 \mathrm{H}), 6.90$ (md, $J=8.9 \mathrm{~Hz}$, $2 \mathrm{H}), \quad 3.87(\mathrm{~s}, 3 \mathrm{H}) \cdot{ }^{13} \mathrm{C}-\mathrm{NMR}\left(75 \mathrm{MHz}, \mathrm{CDCl}_{3}\right): \delta=159.1,140.8$, 133.8, 128.7, 128.1, 126.7, 126.6, 114.2, 55.3. IR (KBr): $3066,3034,2963,1605,1489,1289,1252,1202,1036,834$, 762, $574 \mathrm{~cm}^{-1}$. MS (EI), $\mathrm{m} / \mathrm{z}$ (relative intensity) 184 (100) $\left[\mathrm{M}^{+}\right], 169(41), 141$ (29), 115 (13), 89 (2), 63 (1). HR-MS (EI) $\mathrm{m} / \mathrm{z}$ calcd for $\mathrm{C}_{13} \mathrm{H}_{12} \mathrm{O} 184.0888$, found 184.0920 .

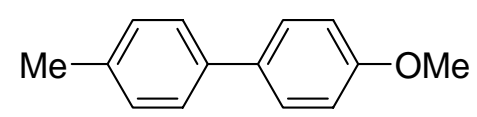

\section{4-Methoxy-4'-methylbiphenyl (Table 2, entry 2):}

The representative procedure was followed, using [Pd(dba)2] (14.4 mg, $0.025 \mathrm{mmol}, 2.5 \mathrm{~mol} \%), 12$ (8.2 mg, $0.050 \mathrm{mmol}$, 5.0 mol\%), 4-methoxyphenylmagnesiumbromide $(0.5 \mathrm{M}$ in $\mathrm{THF}$, $3.0 \mathrm{~mL}, \quad 1.50 \mathrm{mmol})$ and 4-methylphenyltosylate (263 mg, $1.00 \mathrm{mmol})$. After $22 \mathrm{~h}$, purification by chromatography (npentane $\left./ \mathrm{Et}_{2} \mathrm{O}=1 / 0 \rightarrow 200 / 1\right)$ yielded 4-methoxy-4'-methylbiphenyl (186 mg, 0.94 mmol, $94 \%)$ as a colourless solid (mp: 107.9108.1 $\left.{ }^{\circ} \mathrm{C}\right)$. The spectral data were in accordance with those reported in the literature. ${ }^{3}$ 
${ }^{1} \mathrm{H}-\mathrm{NMR} \quad\left(300 \mathrm{MHz}, \mathrm{CDCl}_{3}\right): \delta=7.52$ (md, $\left.J=8.8 \mathrm{~Hz}, 2 \mathrm{H}\right), 7.46$ (md, $J=8.0 \mathrm{~Hz}, 2 \mathrm{H}), 7.22(\mathrm{md}, J=7.9 \mathrm{~Hz}, 2 \mathrm{H}), 7.00(\mathrm{md}, J=$ $8.8 \mathrm{~Hz}, 2 \mathrm{H}), 3.85(\mathrm{~s}, 3 \mathrm{H}), 2.39(\mathrm{~s}, 3 \mathrm{H}) .{ }^{13} \mathrm{C}-\mathrm{NMR}(75 \mathrm{MHz}$, $\left.\mathrm{CDCl}_{3}\right): \delta=158.9,137.9,136.3,133.7,129.4,127.9,126.6$, 114.1, 55.3, 21.0. IR (KBr): 2536, 1894, 1608, 1501, 1377, 1289, 1251, 1137, 1039, 809, 648, $499 \mathrm{~cm}^{-1} . \mathrm{MS}(\mathrm{EI}), \mathrm{m} / \mathrm{z}$ (relative intensity) $198(100)\left[\mathrm{M}^{+}\right], 183(35), 156$ (2), 128 (2), 99 (2), 77 (1). HR-MS (EI) $\mathrm{m} / z$ calcd for $\mathrm{C}_{14} \mathrm{H}_{14} \mathrm{O}$ 198.1045, found 198.1051 .

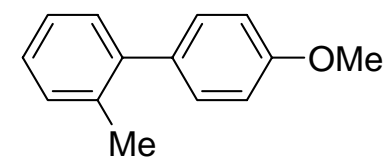

\section{4-Methoxy-2'-methylbiphenyl (Table 2, entry 3):}

The representative procedure was followed, using [Pd(dba) 2 ] (14.4 mg, $0.025 \mathrm{mmol}, 2.5 \mathrm{~mol} \%), 12$ (8.2 mg, $0.050 \mathrm{mmol}$, 5.0 mol\%), 4-methoxyphenylmagnesiumbromide $(0.5 \mathrm{M}$ in $\mathrm{THF}$, $3.0 \mathrm{~mL}, \quad 1.50 \mathrm{mmol})$ and 2-methylphenyltosylate (263 mg, $1.00 \mathrm{mmol})$. After $22 \mathrm{~h}$, purification by chromatography (npentane $/ \mathrm{Et}_{2} \mathrm{O}=$ 200/1) yielded 4-methoxy-2'-methylbiphenyl (192 mg, $0.92 \mathrm{mmol}, 92 \%$ as a light yellow liquid. The spectral data were in accordance with those reported in the literature. ${ }^{4}$

${ }^{1} \mathrm{H}-\mathrm{NMR} \quad\left(300 \mathrm{MHz}, \mathrm{CDCl}_{3}\right): \delta=7.25-7.21(\mathrm{~m}, 6 \mathrm{H}), 6.95(\mathrm{md}, J=$ $8.8 \mathrm{~Hz}, 2 \mathrm{H}), 3.85(\mathrm{~s}, 3 \mathrm{H}), 2.27(\mathrm{~s}, 3 \mathrm{H}) .{ }^{13} \mathrm{C}-\mathrm{NMR} \quad(75 \mathrm{MHz}$, $\left.\mathrm{CDCl}_{3}\right): \delta=158.5,141.5,135.5,134.4,130.3,130.2,129.9$, 126.9, 125.7, 113.5, 55.3, 20.5. IR (KBr): 2935, 2043, 1613, 
$1484,1380,1244,1107,1039,866,834,761,636,620 \mathrm{~cm}^{-1}$. MS (EI), $\mathrm{m} / \mathrm{z}$ (relative intensity) $198(100)\left[\mathrm{M}^{+}\right], 183(18), 155$ (14), 128 (7), 55 (2). HR-MS (EI) $\mathrm{m} / \mathrm{z}$ calcd for $\mathrm{C}_{14} \mathrm{H}_{14} \mathrm{O}$ 198.1045, found 198.1028.

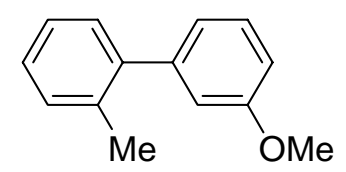

\section{3'-Methoxy-2-methylbiphenyl (Table 2, entry 4):}

The representative procedure was followed, using [Pd(dba) 2 ] (14.4 mg, $0.025 \mathrm{mmol}, 2.5 \mathrm{~mol} \%), 12$ (8.2 mg, $0.050 \mathrm{mmol}$, 5.0 mol\%), 3-methoxyphenylmagnesiumbromide $(1.0 \mathrm{M}$ in $\mathrm{THF}$, $1.5 \mathrm{~mL}, \quad 1.50 \mathrm{mmol})$ and 2-methylphenyltosylate (263 mg, $1.00 \mathrm{mmol})$. After $22 \mathrm{~h}$, purification by chromatography (npentane $/ \mathrm{Et}_{2} \mathrm{O}=200 / 1$ ) yielded $\quad 3$-methoxy-2-methylbiphenyl (170 mg, $0.86 \mathrm{mmol}, 86 \%$ as a light yellow liquid. The spectral data were in accordance with those reported in the literature. ${ }^{5}$

${ }^{1} \mathrm{H}-\mathrm{NMR} \quad\left(300 \mathrm{MHz}, \mathrm{CDCl}_{3}\right): \delta=7.38-7.24(\mathrm{~m}, 5 \mathrm{H}), 6.96-6.88(\mathrm{~m}$, $3 \mathrm{H}), \quad 3.85(\mathrm{~s}, 3 \mathrm{H}), 2.31(\mathrm{~s}, 3 \mathrm{H}) \cdot{ }^{13} \mathrm{C}-\mathrm{NMR} \quad\left(75 \mathrm{MHz}, \mathrm{CDCl}_{3}\right):$ $\delta=159.3,143.4,141.8,135.3,130.3,129.6,129.0,127.3$, 125.7, 121.7, 114.8, 112.3, 55.2, 20.4. IR (KBr): 2954, 2833, $1580,1475,1418,1316,1276,1210,1177,1045,1021,856$, 782, $720 \mathrm{~cm}^{-1}$. MS (EI), $\mathrm{m} / \mathrm{z}$ (relative intensity) 198 (100) $\left[\mathrm{M}^{+}\right], 167$ (48), 155 (16), 128 (9). HR-MS (EI) m/z calcd for $\mathrm{C}_{14} \mathrm{H}_{14} \mathrm{O}$ 198.1045, found 198.1032 . 


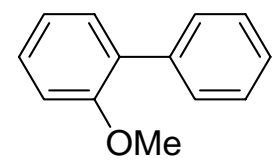

2-Methoxybiphenyl (Table 2, entry 5):

The representative procedure was followed, using [Pd(dba) ${ }_{2}$ (14.4 mg, $0.025 \mathrm{mmol}, 2.5 \mathrm{~mol} \%), 12$ (8.2 mg, $0.050 \mathrm{mmol}$, 5.0 mol\%), phenylmagnesiumchloride $(1.5 \mathrm{M}$ in $\mathrm{THF}, 1.0 \mathrm{~mL}$, $1.50 \mathrm{mmol})$ and 2-methoxyphenyltosylate (279 $\mathrm{mg}, 1.00 \mathrm{mmol})$. After $22 \mathrm{~h}$, purification by chromatography ( $n$-pentane/Et ${ }_{2} \mathrm{O}=$ $200 / 1$ ) yielded 2-methoxybiphenyl (171 mg, $0.93 \mathrm{mmol}, 93$ \%) as a light yellow liquid. The spectral data were in accordance with those reported in the literature. ${ }^{6}$

${ }^{1} \mathrm{H}-\mathrm{NMR} \quad\left(300 \mathrm{MHz}, \mathrm{CDCl}_{3}\right): \delta=7.55$ (md, $\left.J=8.2 \mathrm{~Hz}, 2 \mathrm{H}\right), 7.43$ (td, $J=7.3,0.9 \mathrm{~Hz}, 2 \mathrm{H}), 7.37-7.32(\mathrm{~m}, 3 \mathrm{H}), 7.05$ (td, $J=$ $7.3,0.9 \mathrm{~Hz}, 1 \mathrm{H}), 7.00(\mathrm{~d}, J=8.8 \mathrm{~Hz}, 1 \mathrm{H}), 3.82(\mathrm{~s}, 3 \mathrm{H}) .{ }^{13} \mathrm{C}-$ $\operatorname{NMR}\left(75 \mathrm{MHz}, \mathrm{CDCl}_{3}\right): \delta=156.5,138.5,130.9,130.7,129.5$, $128.6,127.9,126.9,120.8,111.2,55.5 . \operatorname{IR}(\mathrm{KBr}): 3791,2935$, 2833, 1596, 1503, 1481, 1462, 1429, 1257, 1179, 1054, 1025, $799 \mathrm{~cm}^{-1}$. MS (EI), $\mathrm{m} / \mathrm{z}$ (relative intensity) $184(100)\left[\mathrm{M}^{+}\right], 169$ (46), 152 (6), 141 (28), 115 (23), 76 (3). HR-MS (EI) m/z calcd for $\mathrm{C}_{13} \mathrm{H}_{12} \mathrm{O} 184.0888$, found 184.0885 . 


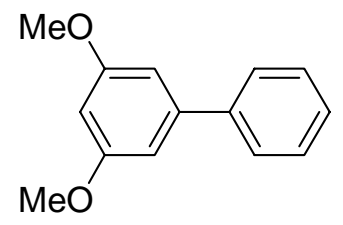

\section{3,5-Dimethoxybiphenyl (Table 2, entry 6):}

The representative procedure was followed, using [Pd(dba) 2 ] (14.4 mg, $0.025 \mathrm{mmol}, 2.5 \mathrm{~mol} \%), 12$ (8.2 mg, $0.050 \mathrm{mmol}$, $5.0 \mathrm{~mol} \%)$, phenylmagnesiumchloride $(1.5 \mathrm{M}$ in THF, $1.0 \mathrm{~mL}$, $1.50 \mathrm{mmol})$ and 3,5-dimethoxyphenyltosylate (309 $\mathrm{mg}$, $1.00 \mathrm{mmol})$. After $22 \mathrm{~h}$, purification by chromatography ( $n-$ pentane $\left./ \mathrm{Et}_{2} \mathrm{O}=200 / 1 \rightarrow 100 / 1\right) \quad$ yielded $\quad 3$, -dimethoxybiphenyl (205 mg, $0.95 \mathrm{mmol}, 95$ \%) as a colourless liquid. The spectral data were in accordance with those reported in the literature. ${ }^{7}$ ${ }^{1} \mathrm{H}-\mathrm{NMR} \quad\left(300 \mathrm{MHz}, \mathrm{CDCl}_{3}\right): \delta=7.58-7.62(\mathrm{~m}, 2 \mathrm{H}), 7.34-7.47(\mathrm{~m}$, $3 \mathrm{H}), 6.76(\mathrm{~d}, J=2.3 \mathrm{~Hz}, 2 \mathrm{H}), 6.50(\mathrm{t}, J=2.3 \mathrm{~Hz}, 1 \mathrm{H}), 3.86$ $(\mathrm{s}, 6 \mathrm{H}) \cdot{ }^{13} \mathrm{C}-\mathrm{NMR} \quad\left(75 \mathrm{MHz}, \mathrm{CDCl}_{3}\right): \delta=161.0,143.5,141.2$, 128.7, 127.5, 127.2, 105.5, 99.3, 55.4. IR (KBr): 3059, 2937, 2837, 1591, 1574, 1499, 1415, 1334, 1202, 1150, 1063, $914 \mathrm{~cm}^{-1}$. MS (EI) $\mathrm{m} / \mathrm{z}$ (relative intensity) $214(100)\left[\mathrm{M}^{+}\right], 185$ (21), 170 (8), 155 (4), 141 (8), 128 (13), 115 (5). HR-MS (EI) m/z calcd for $\mathrm{C}_{14} \mathrm{H}_{14} \mathrm{O}_{2} 214.0994$, found 214.0973 . 


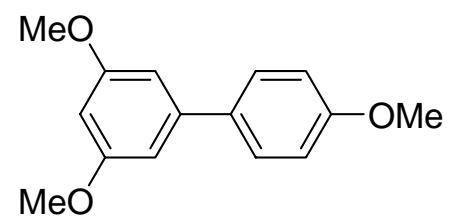

\section{3,5,4'-Trimethoxybiphenyl (Table 2, entry 7):}

The representative procedure was followed, using [Pd(dba) 2] (14.4 mg, $0.025 \mathrm{mmol}, 2.5 \mathrm{~mol} \%), 12$ (8.2 mg, $0.050 \mathrm{mmol}$, 5.0 mol은, 4-methoxyphenylmagnesiumbromide $(0.5 \mathrm{M}$ in $\mathrm{THF}$, $3.0 \mathrm{~mL}, 1.50 \mathrm{mmol})$ and 3,5-dimethoxyphenyltosylate (309 mg, $1.00 \mathrm{mmol})$. After $22 \mathrm{~h}$, purification by chromatography ( $n-$ pentane $/ \mathrm{Et}{ }_{2} \mathrm{O}=200 / 1 \rightarrow 25 / 1$ ) yielded 3,5,4'-trimethoxybiphenyl (233 mg, $0.95 \mathrm{mmol}, 95$ \%) as a bright yellow solid (mp: 61.3$\left.61.5^{\circ} \mathrm{C}\right)$. The spectral data were in accordance with those reported in the literature. ${ }^{8}$

${ }^{1} \mathrm{H}-\mathrm{NMR} \quad\left(300 \mathrm{MHz}, \mathrm{CDCl}_{3}\right): \delta=7.55$ (md, $\left.J=2.9 \mathrm{~Hz}, 1 \mathrm{H}\right), 7.52$ (md, $J=2.9 \mathrm{~Hz}, 1 \mathrm{H}), 7.00(\mathrm{md}, J=2.9 \mathrm{~Hz}, 1 \mathrm{H}), 6.97$ (md, $J=$ $2.9 \mathrm{~Hz}, 1 \mathrm{H}), 6.72(\mathrm{~d}, J=2.4 \mathrm{~Hz}, 2 \mathrm{H}), 6.45$ (md, $J=2.4 \mathrm{~Hz}$, $1 \mathrm{H}), \quad 3.86(\mathrm{~s}, 9 \mathrm{H}) \cdot{ }^{13} \mathrm{C}-\mathrm{NMR}\left(75 \mathrm{MHz}, \mathrm{CDCl}_{3}\right): \delta=161.0,159.3$, $143.0,133.6,128.1,114.1,105.0,98.7,55.3,55.2$. IR (KBr): $3078,1996,1834,1576,1515,1440,1349,1292,1206,1150$, 1027, 991, 860, $810 \mathrm{~cm}^{-1}$. MS (EI), $\mathrm{m} / \mathrm{z}$ (relative intensity) 244 (100) $\left[\mathrm{M}^{+}\right], 229(14), 201(12), 171(4), 155$ (3), 115 (7) . HRMS (EI) $\mathrm{m} / \mathrm{z}$ calcd for $\mathrm{C}_{15} \mathrm{H}_{16} \mathrm{O}_{3} 244.1099$, found 244.1075 . 
<smiles>COc1cc(OC)cc(-c2ccccc2OC)c1</smiles>

3, 5,2'-Trimethoxybiphenyl (Table 2, entry 8):

The representative procedure was followed, using [Pd(dba) 2$]$ (14.4 mg, $0.025 \mathrm{mmol}, 2.5 \mathrm{~mol} \%), 12$ (8.2 mg, $0.050 \mathrm{mmol}$, $5.0 \mathrm{~mol} \%), 2$-methoxyphenylmagnesiumbromide $(1.0 \mathrm{M}, \quad 1.5 \mathrm{~mL}$, $1.50 \mathrm{mmol}$ ) and 3,5-dimethoxyphenyltosylate (309 $\mathrm{mg}$, $1.00 \mathrm{mmol})$. After $22 \mathrm{~h}$, purification by chromatography ( $n-$ pentane $\left./ \mathrm{Et}_{2} \mathrm{O}=200 / 1 \rightarrow 50 / 1\right)$ yielded $3, \mathbf{5}, \mathbf{2}^{\prime}$-trimethoxybiphenyl (227 mg, $0.93 \mathrm{mmol}, 93 \%)$ as a yellow oil.

${ }^{1} \mathrm{H}-\mathrm{NMR} \quad\left(300 \mathrm{MHz}, \mathrm{CDCl}_{3}\right): \delta=7.38-7.28(\mathrm{~m}, 2 \mathrm{H}), 7.07-6.96(\mathrm{~m}$, $2 \mathrm{H}), 6.68(\mathrm{~d}, J=2.38,2 \mathrm{H}), 6.47(\mathrm{dd}, J=2.38,1 \mathrm{H}), 3.83(\mathrm{~s}$, $6 \mathrm{H}), \quad 3.82(\mathrm{~s}, 3 \mathrm{H}) \cdot{ }^{13} \mathrm{C}-\mathrm{NMR}\left(75 \mathrm{MHz}, \mathrm{CDCl}_{3}\right): \delta=160.3,160.3$, 156.4, 140.4, 130.7, 130.6, 128.7, 120.7, 111.2, 107.8, 99.2, 55.5, 55.3, 55.3. IR (KBr): 3053, 2999, 2933, 1910, 1592, 1499, 1416, 1331, 1202, 1178, 1149, 1064, 1013, 925, 851, 809 $\mathrm{cm}^{-1}$. MS (EI), $\mathrm{m} / \mathrm{z}$ (relative intensity) 244 (100) $\left[\mathrm{M}^{+}\right], 229$ $(18), 213(52), 198(10), 171(14), 155 \quad(7), 125$ (7), 115 (16) . HR-MS (EI) $\mathrm{m} / \mathrm{z}$ calcd for $\mathrm{C}_{15} \mathrm{H}_{16} \mathrm{O}_{3} 244.1099$, found 244.1096<smiles>COc1ccc(-c2ccc(F)cc2)cc1</smiles>

\section{4-Fluoro-4'-methoxybiphenyl (Table 3, entry 1):}

The representative procedure was followed, using [Pd(dba) 2] (14.4 mg, $0.025 \mathrm{mmol}, 2.5 \mathrm{~mol} \%), 12$ (8.2 mg, $0.050 \mathrm{mmol}$, 
5.0 molo), 4-methoxyphenylmagnesiumbromide $(0.5 \mathrm{M}$ in $\mathrm{THF}$, $3.0 \mathrm{~mL}, \quad 1.50 \mathrm{mmol})$ and 4-fluorophenyltosylate $(267 \mathrm{mg}$, $1.00 \mathrm{mmol})$. After $22 \mathrm{~h}$, purification by chromatography ( $n-$ pentane/Et ${ }_{2} \mathrm{O}=$ 200/1) yielded 4-fluoro-4'-methoxybiphenyl (188 mg, $0.93 \mathrm{mmol}, 93$ 으) as a colourless solid (mp: 87.3$\left.87.8^{\circ} \mathrm{C}\right)$. The spectral data were in accordance with those reported in the literature. ${ }^{9}$

${ }^{1} \mathrm{H}-\mathrm{NMR} \quad\left(400 \mathrm{MHz}, \mathrm{CDCl}_{3}\right): \delta=7.53-7.46(\mathrm{~m}, 4 \mathrm{H}), 7.10(\mathrm{dd}, J=$ $8.6,8.7 \mathrm{~Hz}, 2 \mathrm{H}), 6.97(\mathrm{dd}, J=8.8,8.7 \mathrm{~Hz}, 2 \mathrm{H}), 3.85$ (s, 3H). ${ }^{13} \mathrm{C}-\mathrm{NMR} \quad\left(75 \mathrm{MHz}, \mathrm{CDCl}_{3}\right): \delta=161.9 \quad(\mathrm{~d}, J=244.2 \mathrm{~Hz})$, 158.9, $136.7(\mathrm{~d}, J=3.5 \mathrm{~Hz}), 132.5,128.0(\mathrm{~d}, J=8.0 \mathrm{~Hz})$, 127.8, 115.3, $(\mathrm{d}, J=21.1 \mathrm{~Hz}), 114.1,55.2 .{ }^{19} \mathrm{~F}-\mathrm{NMR}(282 \mathrm{MHz}$, $\left.\mathrm{CDCl}_{3}\right):-117.15$ (m). IR (KBr): 3790, 3015, 2965, 2842, 1599, $1498,1441,1326,1289,1231,1181,1037,824,789 \mathrm{~cm}^{-1} . \mathrm{MS}$ (EI), $\mathrm{m} / \mathrm{z}$ (relative intensity) $202(100)\left[\mathrm{M}^{+}\right], 187$ (55), 159 (65), 133 (46). HR-MS (EI) $\mathrm{m} / z$ calcd for $\mathrm{C}_{13} \mathrm{H}_{11} \mathrm{FO} 202.0794$, found 202.0790 .

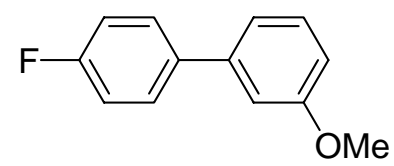

\section{4-Fluoro-3'-methoxybiphenyl (Table 3, entry 2):}

The representative procedure was followed, using [Pd(dba) 2$]$ (14.4 mg, $0.025 \mathrm{mmol}, 2.5 \mathrm{~mol} \%), 12$ (8.2 mg, $0.050 \mathrm{mmol}$, 5.0 mol\%), 3-methoxyphenylmagnesiumbromide $\quad(1.0 \mathrm{M}, \quad 1.5 \mathrm{~mL}$, $1.50 \mathrm{mmol})$ and 4-fluorophenyltosylate (267 mg, $1.00 \mathrm{mmol})$. 
After $22 \mathrm{~h}$, purification by chromatography ( $n$-pentane/Et ${ }_{2} \mathrm{O}=$ $200 / 1)$ yielded f-Fluoro-3'-methoxybiphenyl (158 mg, $0.78 \mathrm{mmol}$, $78 \%)$ as a light yellow oil. The spectral data were in accordance with those reported in the literature. ${ }^{10}$

${ }^{1} \mathrm{H}-\mathrm{NMR} \quad\left(400 \mathrm{MHz}, \quad \mathrm{CDCl}_{3}\right): \delta=7.58-7.51 \quad(\mathrm{~m}, 2 \mathrm{H}), 7.36 \quad(\mathrm{t}, \quad J=$ $7.8 \mathrm{~Hz}, 1 \mathrm{H}), 7.19-7.05(\mathrm{~m}, 4 \mathrm{H}), 6.93-6.88(\mathrm{~m}, 1 \mathrm{H}), 3.87$ (s, $3 \mathrm{H}) \cdot{ }^{13} \mathrm{C}-\mathrm{NMR} \quad\left(75 \mathrm{MHz}, \mathrm{CDCl}_{3}\right): \delta=162.5 \quad(\mathrm{~d}, \quad J=246.7 \mathrm{~Hz})$, 159.9, 141.7, 137.2, 129.8, $129.7(d, J=64.8 \mathrm{~Hz}), 128.7(\mathrm{~d}$, $J=8.1 \mathrm{~Hz}), 119.5,115.6(\mathrm{~d}, J=21.4 \mathrm{~Hz}), 112.6(\mathrm{~d}, J=$ $28.7 \mathrm{~Hz}), \quad 55.3 .{ }^{19} \mathrm{~F}-\mathrm{NMR}\left(282 \mathrm{MHz}, \mathrm{CDCl}_{3}\right):-115.98 \quad(\mathrm{~m}) \cdot \mathrm{IR}$ $(\mathrm{KBr}): 3041,2957,2939,1965,1600,1576,1514,1480,1295$, 1218, 1170, 1096, 868, $775 \mathrm{~cm}^{-1}$. MS (EI), $\mathrm{m} / \mathrm{z}$ (relative intensity) $202(100)\left[\mathrm{M}^{+}\right], 187(50), 159$ (69), 133 (41). HR-MS (EI) $\mathrm{m} / \mathrm{z}$ calcd for $\mathrm{C}_{13} \mathrm{H}_{11} \mathrm{FO} 202.0794$, found 202.0795 .

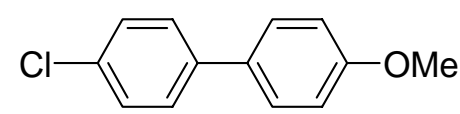

\section{4-Chloro-4'-methoxybiphenyl (Table 3, entry 3):}

The representative procedure was followed, using [Pd(dba) 2$]$ (14.4 mg, $0.025 \mathrm{mmol}, 2.5 \mathrm{~mol} \%), 12$ (8.2 mg, $0.050 \mathrm{mmol}$, 5.0 molo), 4-methoxyphenylmagnesiumbromide $(0.5 \mathrm{M}$ in $\mathrm{THF}$, $3.0 \mathrm{~mL}, \quad 1.50 \mathrm{mmol})$ and 4-chlorophenyltosylate $(283 \mathrm{mg}$, $1.00 \mathrm{mmol})$. After $22 \mathrm{~h}$, purification by chromatography (npentane $/ \mathrm{Et}_{2} \mathrm{O}=200 / 1$ ) yielded $\quad$ 4-chloro-4'-methoxybiphenyl (192 mg, $0.88 \mathrm{mmol}, 88 \%$ as a colourless solid (mp: 111.1- 
$\left.111.3^{\circ} \mathrm{C}\right)$. The spectral data were in accordance with those reported in the literature. ${ }^{11}$

${ }^{1} \mathrm{H}-\mathrm{NMR} \quad\left(600 \mathrm{MHz}, \mathrm{CDCl}_{3}\right): \delta=7.52-7.50(\mathrm{~m}, 4 \mathrm{H}), 7.41-7.36 \quad(\mathrm{~m}$, $2 \mathrm{H}), 6.93(\mathrm{~d}, J=8.8 \mathrm{~Hz}, 2 \mathrm{H}), 3.86(\mathrm{~s}, 3 \mathrm{H}) .{ }^{13} \mathrm{C}-\mathrm{NMR} \quad(75 \mathrm{MHz}$, $\left.\mathrm{CDCl}_{3}\right): \delta=159.4,139.3,132.7,132.5,128.8,128.0,127.9$, 114.3, 55.3. IR $(\mathrm{KBr}): 3790,3011,2839,1603,1580,1522$, $1481,1395,1287,1297,1179,1036,998,819 \mathrm{~cm}^{-1} . M S(E I), \mathrm{m} / z$ (relative intensity) 221 (5), 220 (28), $218(100)\left[\mathrm{M}^{+}\right], 205$ $(13), 204(5), 203 \quad(46), 177 \quad(12), 176 \quad(5), 175 \quad(41), 149$ (11), 139 (21). HR-MS (EI) $\mathrm{m} / \mathrm{z}$ calcd for $\mathrm{C}_{13} \mathrm{H}_{11} \mathrm{ClO} 218.0498$, found 218.0482 .

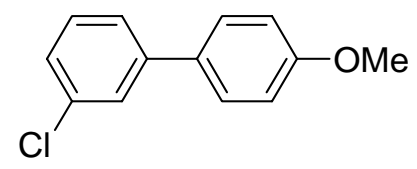

\section{3-Chloro-4'-methoxybiphenyl (Table 3, entry 4):}

The representative procedure was followed, using [Pd(dba) 2$]$ (14.4 mg, $0.025 \mathrm{mmol}, 2.5 \mathrm{~mol} \%), 12$ (8.2 mg, $0.050 \mathrm{mmol}$, 5.0 molo), 4-methoxyphenylmagnesiumbromide $(0.5 \mathrm{M}$ in $\mathrm{THF}$, $3.0 \mathrm{~mL}, \quad 1.50 \mathrm{mmol})$ and 3-chlorophenyltosylate (283 mg, $1.00 \mathrm{mmol})$. After $22 \mathrm{~h}$, purification by chromatography (npentane $/ \mathrm{Et}{ }_{2} \mathrm{O}=200 / 1$ ) yielded $\quad$ 3-chloro-4'-methoxybiphenyl (191 mg, $0.87 \mathrm{mmol}, 87 \%$ as a colourless solid (mp: 52.352. ' $\left.^{\circ} \mathrm{C}.\right)$. The spectral data were in accordance with those reported in the literature. ${ }^{12}$ 
${ }^{1} \mathrm{H}-\mathrm{NMR} \quad\left(600 \mathrm{MHz}, \mathrm{CDCl}_{3}\right): \delta=7.54(\mathrm{dd}, J=8.0,1.9 \mathrm{~Hz}, 1 \mathrm{H})$, $7.50(\mathrm{md}, J=8.5 \mathrm{~Hz}, 2 \mathrm{H}), 7.44-7.41(\mathrm{~m}, 1 \mathrm{H}), 7.40(\mathrm{dd}, J=$ $8.1,1.9 \mathrm{~Hz}, 1 \mathrm{H}), 7.29-7.26(\mathrm{~m}, 1 \mathrm{H}), 6.98 \quad(\mathrm{md}, J=9.1 \mathrm{~Hz}$, $2 \mathrm{H}), \quad 3.86(\mathrm{~s}, 3 \mathrm{H}) .{ }^{13} \mathrm{C}-\mathrm{NMR}\left(75 \mathrm{MHz}, \mathrm{CDCl}_{3}\right): \delta=142.7,134.6$, 132.3, 129.9, 128.1, 126.8, 126.6, 124.8, 114.3, 113.9, 55.3. IR $(\mathrm{KBr}): 3075,2955,1605,1580,1513,1466,1392,1307$, 1289, 1251, 1182, 1101, 1019, 835, $762 \mathrm{~cm}^{-1}$. MS (EI), $\mathrm{m} / z$ (relative intensity) $218(100)\left[\mathrm{M}^{+}\right], 203(28), 175$ (31), 139 (18), 63 (3). HR-MS (EI) $\mathrm{m} / \mathrm{z}$ calcd for $\mathrm{C}_{13} \mathrm{H}_{11} \mathrm{ClO} 218.0498$, found 218.0490

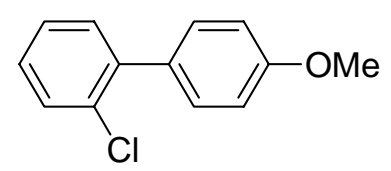

\section{2-Chloro-4'-methoxybiphenyl (Table 3, entry 5):}

The representative procedure was followed, using [Pd(dba) 2 ] (14.4 mg, $0.025 \mathrm{mmol}, 2.5 \mathrm{~mol} \%), 12$ (8.2 mg, $0.050 \mathrm{mmol}$, 5.0 mol\%), 4-methoxyphenylmagnesiumbromide $(0.5 \mathrm{M}$ in $\mathrm{THF}$, $3.0 \mathrm{~mL}, \quad 1.50 \mathrm{mmol})$ and 2-chlorophenyltosylate (283 mg, $1.00 \mathrm{mmol})$. After $22 \mathrm{~h}$, purification by chromatography (npentane $\left./ \mathrm{Et}_{2} \mathrm{O}=\quad 200 / 1 \rightarrow 100 / 1\right) \quad$ yielded $\quad$ 2-chloro-4' methoxybiphenyl (195 mg, $0.89 \mathrm{mmol}, 89 \%)$ as a light yellow oil.

${ }^{1} \mathrm{H}-\mathrm{NMR} \quad\left(300 \mathrm{MHz}, \mathrm{CDCl}_{3}\right): \delta=7.48-7.45 \quad(\mathrm{~m}, 1 \mathrm{H}), 7.40 \quad(\mathrm{md}, \quad J=$ $8.6 \mathrm{~Hz}, 2 \mathrm{H}), 7.35-7.24(\mathrm{~m}, 3 \mathrm{H}), 6.99(\mathrm{md}, \mathrm{J}=8.6 \mathrm{~Hz}, 2 \mathrm{H})$, $3.87(\mathrm{~s}, 3 \mathrm{H}) \cdot{ }^{13} \mathrm{C}-\mathrm{NMR}\left(75 \mathrm{MHz}, \mathrm{CDCl}_{3}\right): \delta=159.1,140.1,132.6$, 
131.8, 131.4, 130.6, 129.9, 128.2, 126.8, 113.5, 55.3. IR $(\mathrm{KBr}): 2934,2835,1610,1578,1514,1465,1439,1294,1153$, 1176, 1073, 1034, 829, 754, $732 \mathrm{~cm}^{-1}$. MS (EI), $\mathrm{m} / \mathrm{z}$ (relative intensity) $220(26), 218(100)\left[\mathrm{M}^{+}\right], 203(21), 175$ (25), 139 (19), 75 (2). HR-MS (EI) $\mathrm{m} / z$ calcd for $\mathrm{C}_{13} \mathrm{H}_{11} \mathrm{ClO} 218.0498$, found 218.0505 .

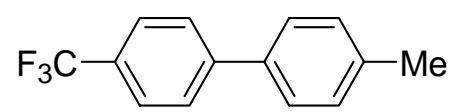

\section{4'-Methyl-4-(trifluoromethyl)biphenyl (Table 3, entry 6):}

The representative procedure was followed, using [Pd(dba) 2$]$ (2.9 mg, $0.005 \mathrm{mmol}, 0.5 \mathrm{~mol} \%), 12$ (1.6 mg, $0.010 \mathrm{mmol}$, 1.0 mol\%), 4-methylphenylmagnesiumbromide $(1.0 \mathrm{M}$ in $\mathrm{THF}$, $1.5 \mathrm{~mL}, \quad 1.50 \mathrm{mmol})$ and 4-(trifluoromethyl)phenyltosylate (317 mg, $1.00 \mathrm{mmol})$. After $22 \mathrm{~h}$, purification by chromatography (n-pentane/Et $\left.{ }_{2} \mathrm{O}=200 / 1\right)$ yielded $\quad \mathbf{4}^{\prime}$-methyl-4(trifluoromethyl)biphenyl (215 mg, $0.91 \mathrm{mmol}, 91 \%$ as a colourless solid (mp: $121.2-121.3^{\circ} \mathrm{C}$ ). The spectral data were in accordance with those reported in the literature. ${ }^{13}$

${ }^{1} \mathrm{H}-\mathrm{NMR} \quad\left(300 \mathrm{MHz}, \mathrm{CDCl}_{3}\right): \delta=7.70-7.67$ (br s, 4H), 7.51 (md, $J=8.2 \mathrm{~Hz}, 2 \mathrm{H}), 7.30-7.26(\mathrm{~m}, 2 \mathrm{H}), 2.43(\mathrm{~s}, 3 \mathrm{H}) .{ }^{13} \mathrm{C}-\mathrm{NMR}$ $\left(75 \mathrm{MHz}, \mathrm{CDCl}_{3}\right): \delta=144.7,138.1,136.9,129.7,129.0 \quad(\mathrm{q}, \mathrm{J}=$ $32.3 \mathrm{~Hz}), 127.2,127.1(\mathrm{~d}, J=4.7 \mathrm{~Hz}), 125.7(\mathrm{q}, J=3.8 \mathrm{~Hz})$, $125.6(q, J=272.2 \mathrm{~Hz}), 21.1 .{ }^{19} \mathrm{~F}-\mathrm{NMR}\left(282 \mathrm{MHz}, \mathrm{CDCl}_{3}\right):-67.78$ (s). IR $(\mathrm{KBr}): 3035,2923,2863,1910,1798,1615,1565,1501$, 
1398, 1322, 1202, 1140, 1071, 851, $809 \mathrm{~cm}^{-1}$. MS (EI), $\mathrm{m} / \mathrm{z}$ (relative intensity) $236(100)\left[\mathrm{M}^{+}\right], 217(7), 167$ (37), 152 (7), 115 (2), 91 (3). HR-MS (EI) $\mathrm{m} / \mathrm{z}$ calcd for $\mathrm{C}_{14} \mathrm{H}_{11} \mathrm{~F}_{3}$ 236.0813 , found 236.0810 .<smiles>COc1ccc(-c2ccc(C(F)(F)F)cc2)cc1</smiles>

4'-Methoxy-4-(trifluoromethyl)biphenyl (Table 3, entry 7):

The representative procedure was followed, using [Pd(dba) 2] (2.9 mg, $0.005 \mathrm{mmol}, 0.5 \mathrm{~mol} \%), 12$ (1.6 mg, $0.010 \mathrm{mmol}$, 1.0 mol\%), 4-methoxyphenylmagnesiumbromide $(0.5 \mathrm{M}$ in $\mathrm{THF}$, $3.0 \mathrm{~mL}, \quad 1.50 \mathrm{mmol})$ and 4-(trifluoromethyl)phenyltosylate (317 mg, $1.00 \mathrm{mmol})$. After $22 \mathrm{~h}$, purification by chromatography (n-pentane/Et $\left.{ }_{2} \mathrm{O}=200 / 1\right) \quad$ yielded $\quad \mathbf{4}^{\prime}$-methoxy-4(trifluoromethyl)biphenyl (232 mg, $0.92 \mathrm{mmol}, 92$ \%) as a colourless solid (mp: 124.1-124.5 $\left.{ }^{\circ} \mathrm{C}.\right)$. The spectral data were in accordance with those reported in the literature. ${ }^{14}$

${ }^{1} \mathrm{H}-\mathrm{NMR} \quad\left(300 \mathrm{MHz}, \mathrm{CDCl}_{3}\right): \delta=7.70-7.62$ (br s, 4H), $7.55(\mathrm{md}, \mathrm{J}=$ $9.1 \mathrm{~Hz}, 2 \mathrm{H}), 7.01$ (md, $J=9.1 \mathrm{~Hz}, 2 \mathrm{H}), 3.87(\mathrm{~s}, 3 \mathrm{H}) .{ }^{13} \mathrm{C}-\mathrm{NMR}$ $\left(75 \mathrm{MHz}, \mathrm{CDCl}_{3}\right): \delta=159.8,144.3,132.0,128.7 \quad(q, \quad J=$ $32.4 \mathrm{~Hz}), 128.5,127.2,125.6(q, J=3.8 \mathrm{~Hz}), 124.7(q, J=$ 272.2 Hz), 114.4, 55.4. ${ }^{19} \mathrm{~F}-\mathrm{NMR}\left(282 \mathrm{MHz}, \mathrm{CDCl}_{3}\right):-62.73(\mathrm{~s})$. IR $(\mathrm{KBr}): 3031,2966,2553,1669,1601,1532,1499,1325$, 1277, 1166, 1119, 1073, 999, 827, $701 \mathrm{~cm}^{-1}$. MS (EI), m/z (relative intensity) $252(100)\left[\mathrm{M}^{+}\right], 237$ (23), 209 (57), 183 
(10), 159 (4). HR-MS (EI) $\mathrm{m} / \mathrm{z}$ calcd for $\mathrm{C}_{14} \mathrm{H}_{11} \mathrm{~F}_{3} \mathrm{O}$ 252.0762, found 252.0774 .

乐

4'-Methyl-3-(trifluoromethyl)biphenyl (Table 3, entry 8):

The representative procedure was followed, using [Pd(dba)2] (2.9 mg, $0.005 \mathrm{mmol}, 0.5 \mathrm{~mol} \%), 12$ (1.6 mg, $0.010 \mathrm{mmol}$, 1.0 mol\%), 4-methylphenylmagnesiumbromide $(1.0 \mathrm{M}$ in $\mathrm{THF}$, $1.5 \mathrm{~mL}, \quad 1.50 \mathrm{mmol})$ and 3-(trifluoromethyl)phenyltosylate (317 mg, $1.00 \mathrm{mmol})$. After $22 \mathrm{~h}$, purification by chromatography (n-pentane/Et $\left.{ }_{2} \mathrm{O}=1 / 0\right)$ yielded $\quad \mathbf{4}^{\prime}$-methyl-3(trifluoromethyl)biphenyl (206 mg, $\left.0.87 \mathrm{mmol}, 87 \frac{\circ}{0}\right)$ as a colourless liquid. ${ }^{1} \mathrm{H}-\mathrm{NMR} \quad\left(300 \mathrm{MHz}, \mathrm{CDCl}_{3}\right): \delta=7.83$ (br $\mathrm{s}$, $1 \mathrm{H}), 7.79-7.74(\mathrm{~m}, 1 \mathrm{H}), 7.62-7.48(\mathrm{~m}, 4 \mathrm{H}), 7.29(\mathrm{~d}, \quad J=$ $7.9 \mathrm{~Hz}, 2 \mathrm{H}), 2.42(\mathrm{~s}, 3 \mathrm{H}) \cdot{ }^{13} \mathrm{C}-\mathrm{NMR}\left(75 \mathrm{MHz}, \mathrm{CDCl}_{3}\right): \delta=141.9$, 137.9, 131.1 (d, $J=32.1 \mathrm{~Hz}), 130.2,129.7,129.2,127.0$, $124.2(\mathrm{~d}, J=272.0 \mathrm{~Hz}), 123.6(\mathrm{q}, J=4.1 \mathrm{~Hz}), 21.1 .{ }^{19} \mathrm{~F}-\mathrm{NMR}$ (282 $\left.\mathrm{MHz}, \mathrm{CDCl}_{3}\right): \delta=-62.99$ (s).IR (KBr): 3030, 2925, 1901, $1611,1484,1438,1330,1258,1178,1156,1110,1034,1015$, 897, 784, $697 \mathrm{~cm}^{-1}$. MS (EI), $\mathrm{m} / z$ (relative intensity) 236 (100) $\left[\mathrm{M}^{+}\right], 217(7), 165(37), 151$ (3), 115 (3), 91 (11). HR-MS (EI) $\mathrm{m} / \mathrm{z}$ calcd for $\mathrm{C}_{14} \mathrm{H}_{11} \mathrm{~F}_{3} 236.0813$, found 236.0819 . 
<smiles>COc1ccc(-c2cccc(C(F)(F)F)c2)cc1</smiles>

4'-Methoxy-3-(trifluoromethyl)biphenyl (Table 3, entry 9):

The representative procedure was followed, using [Pd(dba) 2$]$
(2.9 mg，
$0.005 \mathrm{mmol}$,
$0.5 \mathrm{~mol} \%)$,
12
(1. $6 \mathrm{mg}$,
$0.010 \mathrm{mmol}$,

1.0 mol\%), 4-methoxyphenylmagnesiumbromide $(0.5 \mathrm{M}$ in $\mathrm{THF}$, 3.0 mL, $1.50 \mathrm{mmol})$ and 3-(trifluoromethyl)phenyltosylate (317 mg, $1.00 \mathrm{mmol})$. After $22 \mathrm{~h}$, purification by chromatography (n-pentane/Et $\left.{ }_{2} \mathrm{O}=1 / 0\right)$ yielded $\quad \mathbf{4}^{\prime}$-methoxy-3(trifluoromethyl)-biphenyl (237 mg, $0.94 \mathrm{mmol}, 94 \%$ as a colourless liquid. The spectral data were in accordance with those reported in the literature. ${ }^{15}$

4'-Methoxy-3-(trifluoromethyl)biphenyl (Table 3, entry 10):

The representative procedure was followed, using [Pd(dba) 2$]$ ( $5.8 \mathrm{mg}, 0.010 \mathrm{mmol}, 1.0 \mathrm{~mol} \%), 12(3.3 \mathrm{mg}, 0.020 \mathrm{mmol}$, $2.0 \mathrm{~mol} \%)$ 4-methoxyphenylmagnesiumbromide $(0.5 \mathrm{M}$ in $\mathrm{THF}$, $3.0 \mathrm{~mL}, \quad 1.50 \mathrm{mmol})$ and 3-(trifluoromethyl)phenyltosylate $(317 \mathrm{mg}, 1.00 \mathrm{mmol})$. After $22 \mathrm{~h}$ at ambient temperature, purification by chromatography (n-pentane/Et $\mathrm{O}_{2}=300 / 1$ ) yielded 4'-methoxy-3-(trifluoromethyl)biphenyl $\quad(240 \mathrm{mg}, \quad 0.95 \mathrm{mmol}$, 95 ㅇ) as a colourless liquid. ${ }^{1} \mathrm{H}-\mathrm{NMR} \quad\left(300 \mathrm{MHz}, \mathrm{CDCl}_{3}\right): \delta=7.83-7.77(\mathrm{~m}, 1 \mathrm{H}), 7.76-7.68 \quad(\mathrm{~m}$, $1 \mathrm{H}), 7.60-7.50(\mathrm{~m}, 4 \mathrm{H}), 7.00(\mathrm{dt}, J=9.0,2.3 \mathrm{~Hz}, 2 \mathrm{H}), 3.87$ $(\mathrm{s}, 3 \mathrm{H}) \cdot{ }^{13} \mathrm{C}-\mathrm{NMR}\left(75 \mathrm{MHz}, \mathrm{CDCl}_{3}\right): \delta=159.7,141.6,132.2,131.2$ $(\mathrm{d}, \mathrm{J}=31.7 \mathrm{~Hz}), \quad 129.9,129.2,128.2, \quad 124.2 \quad(\mathrm{q}, \quad \mathrm{J}=$ 
$272.2 \mathrm{~Hz}), 123.4(q, J=3.8 \mathrm{~Hz}), 123.3(q, J=3.8 \mathrm{~Hz})$, $114.4,55.4 .{ }^{19} \mathrm{~F}-\mathrm{NMR}\left(375 \mathrm{MHz}, \mathrm{CDCl}_{3}\right): \delta=-61.53$ (s). IR (KBr): $3040,3004,2839,1891,1611,1520,1486,1441,1335,1298$, 1249, 1125, 1027, 901, 834, $799 \mathrm{~cm}^{-1}$. MS (EI), $\mathrm{m} / \mathrm{z}$ (relative intensity): $252(100)\left[\mathrm{M}^{+}\right], 209$ (38), 183 (5), 139 (3). HR-MS (EI) $\mathrm{m} / \mathrm{z}$ calcd for $\mathrm{C}_{14} \mathrm{H}_{11} \mathrm{~F}_{3} \mathrm{O} 252.0762$, found 252.0741 .<smiles>COc1ccc(-c2ccccn2)cc1</smiles>

\section{2-(4-Methoxyphenyl) pyridine (Table 4, entry 1):}

The representative procedure was followed, using [Pd(dba) 2 ] (14.4 mg, $0.025 \mathrm{mmol}, 2.5 \mathrm{~mol} \%), 12$ (8.2 mg, $0.050 \mathrm{mmol}$, 5.0 mol\%), 4-methoxyphenylmagnesiumbromide $(0.5 \mathrm{M}$ in $\mathrm{THF}$, $3.0 \mathrm{~mL}, \quad 1.50 \mathrm{mmol})$ and 2-pyridinyltosylate $(250 \mathrm{mg}$, $1.00 \mathrm{mmol})$. After $22 \mathrm{~h}$, purification by chromatography (npentane/Et $\left.{ }_{2} \mathrm{O}=10 / 1 \rightarrow 5 / 1\right)$ yielded 2-(4-methoxyphenyl)pyridine (183 mg, $0.99 \mathrm{mmol}, 99 \%)$ as a light yellow solid (mp.: 53.2$\left.53.5^{\circ} \mathrm{C}\right)$. The spectral data were in accordance with those reported in the literature. ${ }^{16}$

${ }^{1} \mathrm{H}-\mathrm{NMR} \quad\left(600 \mathrm{MHz}, \mathrm{CDCl}_{3}\right): \delta=8.66(\mathrm{ddd}, J=4.1,1.8,1.0 \mathrm{~Hz}$, 1H) $, 7.97(\mathrm{md}, J=8.9 \mathrm{~Hz}, 2 \mathrm{H}), 7.74-7.67(\mathrm{~m}, 2 \mathrm{H}), 7.17$ (ddd, $J=6.9,4.9,1.7 \mathrm{~Hz}, 1 \mathrm{H}), 7.0(\mathrm{md}, J=8.9 \mathrm{~Hz}, 2 \mathrm{H}), 3.86(\mathrm{~s}$, $3 \mathrm{H}) \cdot{ }^{13} \mathrm{C}-\mathrm{NMR} \quad\left(75 \mathrm{MHz}, \mathrm{CDCl}_{3}\right): \delta=160.4,157.1,149.5,136.6$, $132.0,128.1,121.4,119.8,114.1,55.3 . \operatorname{IR}(\mathrm{KBr}): 2558,1768$, $1607,1563,1363,1316,1248,1179,1114,884,840,739,620$, 
$581 \mathrm{~cm}^{-1}$. MS (EI), $\mathrm{m} / \mathrm{z}$ (relative intensity) 185 (100) $\left[\mathrm{M}^{+}\right], 170$ (38), 155 (2), 153 (5), 141 (17), 115 (4), 89 (2), 63 (2) . HRMS (EI) $\mathrm{m} / \mathrm{z}$ calcd for $\mathrm{C}_{12} \mathrm{H}_{11} \mathrm{NO} 185.0841$, found 185.0843 .<smiles>Cc1ccccc1-c1ccccn1</smiles>

\section{2-(2-Methylyphenyl) pyridine (Table 4, entry 2):}

The representative procedure was followed, using [Pd(dba) 2 ] (14.4 mg, $\left.0.025 \mathrm{mmol}, 2.5 \mathrm{~mol} \frac{\circ}{\circ}\right), 12$ (8.2 mg, $0.050 \mathrm{mmol}$, 5.0 mol\%), 2-methylphenylmagnesiumbromide $(1.0 \mathrm{M}$ in $\mathrm{THF}$, $1.5 \mathrm{~mL}, \quad 1.50 \mathrm{mmol})$ and 2-pyridinyltosylate (250 mg, $1.00 \mathrm{mmol})$. After $22 \mathrm{~h}$, purification by chromatography ( $n-$ pentane $\left./ \mathrm{Et}_{2} \mathrm{O}=10 / 1 \rightarrow 5 / 1\right) \quad$ yielded 2-(2-methylphenyl)pyridine (155 mg, $0.92 \mathrm{mmol}, 92$ ㅇ) as a light yellow oil. The spectral data were in accordance with those reported in the literature. ${ }^{16}$

${ }^{1} \mathrm{H}-\mathrm{NMR} \quad\left(300 \mathrm{MHz}, \mathrm{CDCl}_{3}\right): \delta=8.68-8.65(\mathrm{~m}, 1 \mathrm{H}), 7.70(\mathrm{dt}, J=$ $7.6,1.6 \mathrm{~Hz}, 1 \mathrm{H}), 7.36(\mathrm{md}, J=7.9 \mathrm{~Hz}, 2 \mathrm{H}), 7.29-7.18 \quad(\mathrm{~m}$, $4 \mathrm{H}), 2.33(\mathrm{~s}, 3 \mathrm{H}) \cdot{ }^{13} \mathrm{C}-\mathrm{NMR}\left(75 \mathrm{MHz}, \mathrm{CDCl}_{3}\right): \delta=160.0,149.2$, $140.4,136.0,135.7,130.7,129.6,128.2,125.8,124.0,121.5$, 20.2. IR $(\mathrm{KBr}): 3060,3016,2958,2925,1587,1470,1425$, 1150, 1025, $751 \mathrm{~cm}^{-1}$. MS (EI), $\mathrm{m} / \mathrm{z}$ (relative intensity) 169 (27) $\left[\mathrm{M}^{+}\right], 168(100), 167(23), 166(4), 141(3), 140(2), 139$ (3), 115 (2). HR-MS (EI) $\mathrm{m} / \mathrm{z}$ calcd for $\mathrm{C}_{12} \mathrm{H}_{11} \mathrm{~N}$ 169.0891, found 169.0889. 
OMe

\section{3-(4-Methoxyphenyl) pyridine (Table 4, entry 3):}

The representative procedure was followed, using $\mathrm{Pd}(\mathrm{dba})_{2}$ (14.4 mg, $0.025 \mathrm{mmol}, 2.5 \mathrm{~mol} \%), 12$ (8.2 mg, $0.050 \mathrm{mmol}$, $5.0 \mathrm{~mol} \%), 4$-methoxyphenylmagnesiumbromide $(0.5 \mathrm{M}$ in $\mathrm{THF}$, 3.0 mL, $\quad 1.50 \mathrm{mmol})$ and 3-pyridinyltosylate $\quad(250 \mathrm{mg}$, $1.00 \mathrm{mmol})$. After $22 \mathrm{~h}$, purification by chromatography ( $n-$

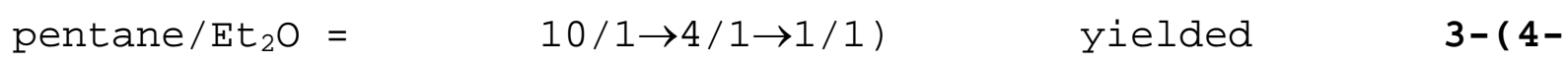
methoxyphenyl)pyridine (182 $\mathrm{mg}, 0.98 \mathrm{mmol}, 98 \%)$ as a light yellow solid (mp: $\left.61.6-61.9^{\circ} \mathrm{C}.\right)$. The spectral data were in accordance with those reported in the literature. ${ }^{17}$

${ }^{1} \mathrm{H}-\mathrm{NMR} \quad\left(300 \mathrm{MHz}, \mathrm{CDCl}_{3}\right): \delta=8.82(\mathrm{dd}, J=2.4,0.8 \mathrm{~Hz}, 1 \mathrm{H})$, $8.55(\mathrm{dd}, J=4.8,1.6 \mathrm{~Hz}, 1 \mathrm{H}), 7.84(\mathrm{ddd}, 7.9,2.4,1.7 \mathrm{~Hz}$, $1 \mathrm{H}), 7.53(\mathrm{md}, J=8.8 \mathrm{~Hz}, 2 \mathrm{H}), 7.35 \quad(\mathrm{ddd}, J=7.9,4.7$, $0.9 \mathrm{~Hz}, 1 \mathrm{H}), 7.02$ (md, $J=8.8 \mathrm{~Hz}, 2 \mathrm{H}), 3.87(\mathrm{~s}, 3 \mathrm{H}) .{ }^{13} \mathrm{C}-\mathrm{NMR}$ $\left(75 \mathrm{MHz}, \mathrm{CDCl}_{3}\right): \delta=159.7,148.0,147.9,136.2,133.8,130.3$, 128.2, 123.4, 114.5, 55.3. IR $(\mathrm{KBr}): 2927,2841,1757,1608$, $1478,1341,1284,1117,1030,947,803,706,642,552 \mathrm{~cm}^{-1} . \mathrm{MS}$ (EI), $\mathrm{m} / \mathrm{z}$ (relative intensity) $185(100)\left[\mathrm{M}^{+}\right], 170(34), 142$ (23), 115 (7), 89 (4), 63 (3). HR-MS (EI) m/z calcd for $\mathrm{C}_{12} \mathrm{H}_{11} \mathrm{NO}$ 185.0841, found 185.0837 . 
$\overbrace{M e}^{1}$

\section{3-(2-Methylphenyl) pyridine (Table 4, entry 4):}

The representative procedure was followed, using [Pd(dba) 2] (14.4 mg, $0.025 \mathrm{mmol}, 2.5 \mathrm{~mol} \%), 12$ (8.2 mg, $0.050 \mathrm{mmol}$, 5.0 mol\%), 2-methylphenylmagnesiumbromide $(1.0 \mathrm{M}$ in $\mathrm{THF}$, $1.5 \mathrm{~mL}, \quad 1.50 \mathrm{mmol})$ and 3-pyridinyltosylate $(250 \mathrm{mg}$, $1.00 \mathrm{mmol})$. After $22 \mathrm{~h}$, purification by chromatography ( $n-$ pentane $\left./ \mathrm{Et}{ }_{2} \mathrm{O}=5 / 1 \rightarrow 2 / 1\right) \quad$ yielded $\quad$ 3-(2-methylphenyl)pyridine (144 mg, $0.85 \mathrm{mmol}, 85 \%$ as a yellow oil. The spectral data were in accordance with those reported in the literature. ${ }^{17}$ ${ }^{1} \mathrm{H}-\mathrm{NMR} \quad\left(300 \mathrm{MHz}, \mathrm{CDCl}_{3}\right): \delta=8.62-8.56(\mathrm{~m}, 2 \mathrm{H}), 7.66(\mathrm{dt}, \mathrm{J}=$ $7.8,1.8 \mathrm{~Hz}, 1 \mathrm{H}), 7.39-7.18(\mathrm{~m}, 5 \mathrm{H}), 2.28(\mathrm{~s}, 3 \mathrm{H}) .{ }^{13} \mathrm{C}-\mathrm{NMR}$ $\left(75 \mathrm{MHz}, \mathrm{CDCl}_{3}\right): \delta=149.8,148.0,138.0,137.5,136.5,135.5$, $130.5,129.8,128.1,126.0,123.0,20.3 . \operatorname{IR}(\mathrm{KBr}): 3401,3023$, $1471,1407,1277,1104,1027,1002,815 \mathrm{~cm}^{-1} . \mathrm{MS}$ (EI), $\mathrm{m} / \mathrm{z}$ (relative intensity) $169(100)\left[\mathrm{M}^{+}\right], 141$ (10), 115 (11), 84 (5) . HR-MS (EI) $\mathrm{m} / \mathrm{z}$ calcd for $\mathrm{C}_{12} \mathrm{H}_{11} \mathrm{~N}$ 169.0891, found 169.0893 . 
$\mathrm{OMe}_{\mathrm{O}}$

6-(2-Methoxyphenyl) quinoline (Table 4, entry 5):

The representative procedure was followed, using [Pd(dba) $\left.{ }_{2}\right]$ (14.4 mg, $0.025 \mathrm{mmol}, 2.5 \mathrm{~mol} \%), 12(8.2 \mathrm{mg}, 0.050 \mathrm{mmol}$, 5.0 mol\%), 2-methoxyphenylmagnesiumbromide $(1.0 \mathrm{M}$ in $\mathrm{THF}$, $1.5 \mathrm{~mL}, 1.50 \mathrm{mmol})$ and 6-quinolyltosylate (300 mg, $1.00 \mathrm{mmol})$. After $22 \mathrm{~h}$, purification by chromatography (n-pentane/Et ${ }_{2} \mathrm{O}=$ $10 / 1 \rightarrow 7 / 1 \rightarrow 5 / 1) \quad$ yielded 6-(2-methoxyphenyl)quinoline (231 mg, 0.98 mmol, 98 \%) as a red oil.

${ }^{1} \mathrm{H}-\mathrm{NMR} \quad\left(400 \mathrm{MHz}, \mathrm{CDCl}_{3}\right): \delta=8.91(\mathrm{dd}, J=4.3,1.4 \mathrm{~Hz}, 1 \mathrm{H})$, $8.22-8.10(\mathrm{~m}, 2 \mathrm{H}), 7.98-7.89(\mathrm{~m}, 2 \mathrm{H}), 7.47-7.35(\mathrm{~m}, 3 \mathrm{H}), 7.13-$ $7.01(\mathrm{~m}, 2 \mathrm{H}), 3.84(\mathrm{~s}, 3 \mathrm{H}) .{ }^{13} \mathrm{C}-\mathrm{NMR}\left(100 \mathrm{MHz}, \mathrm{CDCl}_{3}\right): \delta=156.6$, $150.2,147.4,137.0,136.2,131.8,131.0,129.8,129.1,128.6$, $128.2,127.8,121.1,121.0,111.3,55.6 . \operatorname{IR}(\mathrm{KBr}): 3017,2956$, 2834, 1934, 1597, 1454, 1433, 1355, 1293, 1248, 1118, 1025, 943, 836, $750 \mathrm{~cm}^{-1}$. MS (EI), $\mathrm{m} / \mathrm{z}$ (relative intensity) 235 (100) $\left[\mathrm{M}^{+}\right], 234(42), 220(45), 192(11), 191(12), 165$ (9), 139 (4), 95 (5). HR-MS (EI) $\mathrm{m} / z$ calcd for $\mathrm{C}_{16} \mathrm{H}_{13} \mathrm{NO}$ 235.0997, found 235.0997. 
<smiles>COc1ccc(-c2cccc3cccnc23)cc1</smiles>

\section{8-(4-Methoxyphenyl) quinoline (Table 4, entry 6):}

The representative procedure was followed, using [Pd(dba) 2 ] (14.4 mg, $0.025 \mathrm{mmol}, 2.5 \mathrm{~mol} \%), 12$ (8.2 mg, $0.050 \mathrm{mmol}$, 5.0 mol\%), 2-methoxyphenylmagnesiumbromide $(1.0 \mathrm{M}$ in $\mathrm{THF}$, $1.5 \mathrm{~mL}, 1.50 \mathrm{mmol})$ and 8-quinolyltosylate (300 mg, $1.00 \mathrm{mmol})$. After $22 \mathrm{~h}$, purification by chromatography (n-pentane/Et ${ }_{2} \mathrm{O}=$ $10 / 1 \rightarrow 5 / 1 \rightarrow 3 / 1) \quad$ yielded 8-(4-methoxyphenyl)quinoline $(214 \mathrm{mg}$ 0.91 mmol, $91 \%)$ as a yellow oil with a purity of $93 \%$ as judged by GC-analysis. The spectral data were in accordance with those reported in the literature. ${ }^{18}$

${ }^{1} \mathrm{H}-\mathrm{NMR} \quad\left(300 \mathrm{MHz}, \mathrm{CDCl}_{3}\right): \delta=8.96(\mathrm{dd}, J=3.8,1.4 \mathrm{~Hz}, 1 \mathrm{H})$, $8.20(\mathrm{dd}, J=8.1,1.9 \mathrm{~Hz}, 1 \mathrm{H}), 7.80(\mathrm{dd}, J=8.1,1.4 \mathrm{~Hz}$, $1 \mathrm{H}), 7.72(\mathrm{dd}, J=7.2,1.4 \mathrm{~Hz}, 1 \mathrm{H}), 7.67(\mathrm{md}, J=9.1 \mathrm{~Hz}$, $2 \mathrm{H}), 7.62-7.56(\mathrm{~m}, 1 \mathrm{H}), 7.40(\mathrm{dd}, J=8.1,4.3 \mathrm{~Hz}, 1 \mathrm{H}), 7.06$ (md, $J=8.6 \mathrm{~Hz}, 2 \mathrm{H}), 3.89(\mathrm{~s}, 3 \mathrm{H}) \cdot{ }^{13} \mathrm{C}-\mathrm{NMR}\left(100 \mathrm{MHz}, \mathrm{CDCl}_{3}\right)$ : $\delta=159.0,150.1,146.1,140.2,136.2,131.9,131.7,129.9$, 128.8, 127.1, 126.3, 120.9, 113.6, 55.3. IR (KBr): 3016. 2965, 2835, 1609, 1574, 1494, 1330, 1426, 1292, 1243, 1106, 1031, 966, 816, $767 \mathrm{~cm}^{-1}$. MS (EI), $\mathrm{m} / \mathrm{z}$ (relative intensity) 235 (68) $\left[\mathrm{M}^{+}\right], 234(100), 220(28), 219(20), 191(44), 163(5), 95$ (10). HR-MS (EI) $\mathrm{m} / \mathrm{z}$ calcd for $\mathrm{C}_{16} \mathrm{H}_{13} \mathrm{NO}$ 235.0997, found 235.0973 . 


\section{References}

(1) Lin, G.; Zhang, A. Tetrahedron 2000, 56, 7163-7171.

(2) Lemo, J. ; Heuze, K. ; Astruc, D. Org. Lett. 2005, 7, $2253-$ 2256.

(3) Arvela, R. K.; Leadbeater, N. E.; Sangi, M. S.; Williams, V. A.; Granados, P.; Singer, R. D. J. Org. Chem. 2005, 70, $161-168$.

(4) Barder, T. E.; Buchwald, S. L. Org. Lett. 2004, 6, $2649-$ 2652 .

(5) Lipshutz, B. H.; Siegmann, K.; Garcia, E.; Kayser, F. J. Am. Chem. Soc. 1993, 115, 9276-9282.

(6) Arvela, R. K.; Leadbeater, N. E. Org. Lett. 2005, 7, 21012104

(7) Dol, C. G.; Kamer, P. C. J., van Leeuwen, P. W. N. M. Eur. J. Org. Chem. 1998, 2, 359-364.

(8) Cotterill, P. J.; Owen, P. J.; Scheinmann, F. J. Chem. Soc. Perkin Trans. 1 1974, 2423-2428.

(9) Moore, L. R.; Shaughnessy, K. H. Org. Lett. 2004, 6, 225228 .

(10) Lourak, M.; Vanderesse, R.; Fort, Y.; Caubere, P. J. Org. Chem. 1989, 54, 4844-4848.

(11) Dai, M.; Liang, B.; Wang, C.; Chen, J.; Yang, Z. Org. Lett. 2004, 6, 221-224.

(12) Dewar, J. J. Chem. Soc. 1958, 4265-4269.

(13) Roy, A. H.; Hartwig, J. F. J. Am. Chem. Soc. 2003, 125, $8704-8705$. 
(14) Denmark, S. E.; Ober, M. H. Org. Lett. 2003, 5, 13571360 .

(15) Badone, D.; Baroni, M.; Cardamone, R.; Ielmini, A.; Guzzi, U. J. Org. Chem. 1997, 62, 7170-7173.

(16) Nunez, A.; Sanchez, A.; Burgos, C.; Alvarez-Builla, J. Tetrahedron 2004, 60, 6217-6224.

(17) Feuerstein, M.; Doucet, H.; Santelli, M. J. Organomet. Chem. 2003, 687, 327-336.

(18) Zhang, W.; Chen, C.; Hiu-Tung, L.; Yimin, L.; Nagashima, T. Org. Lett. 2004, 6, 1473-1476. 


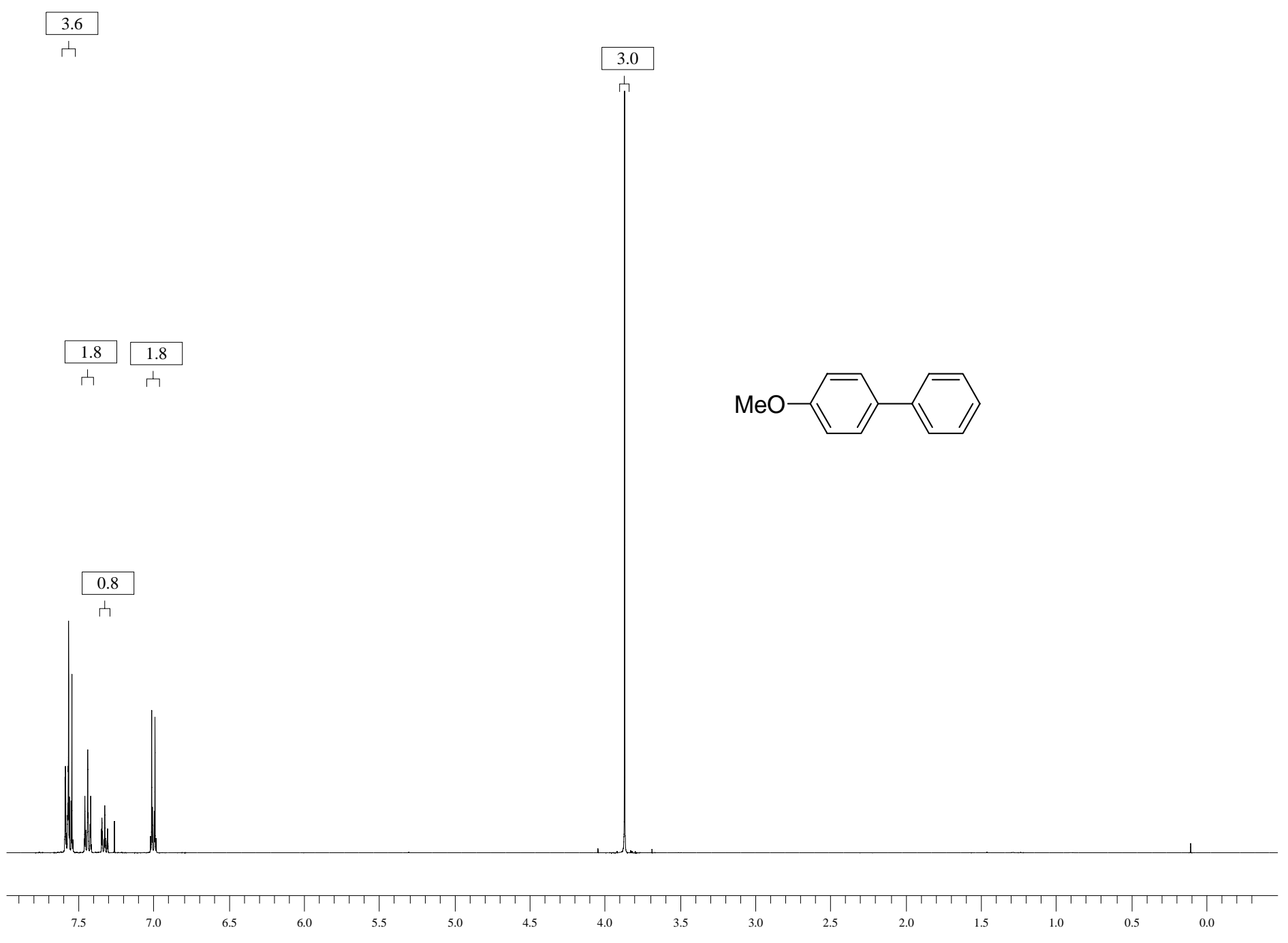




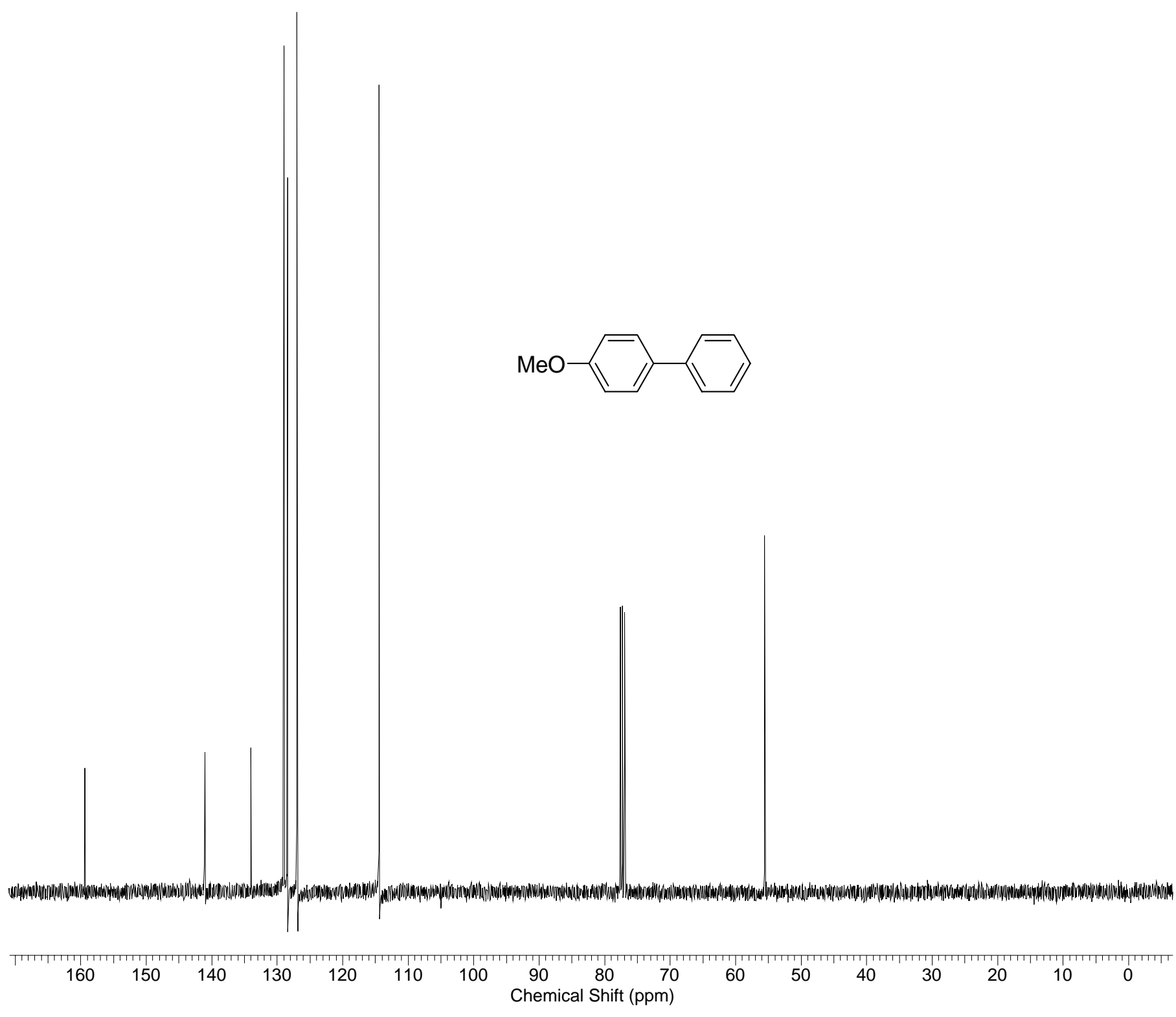



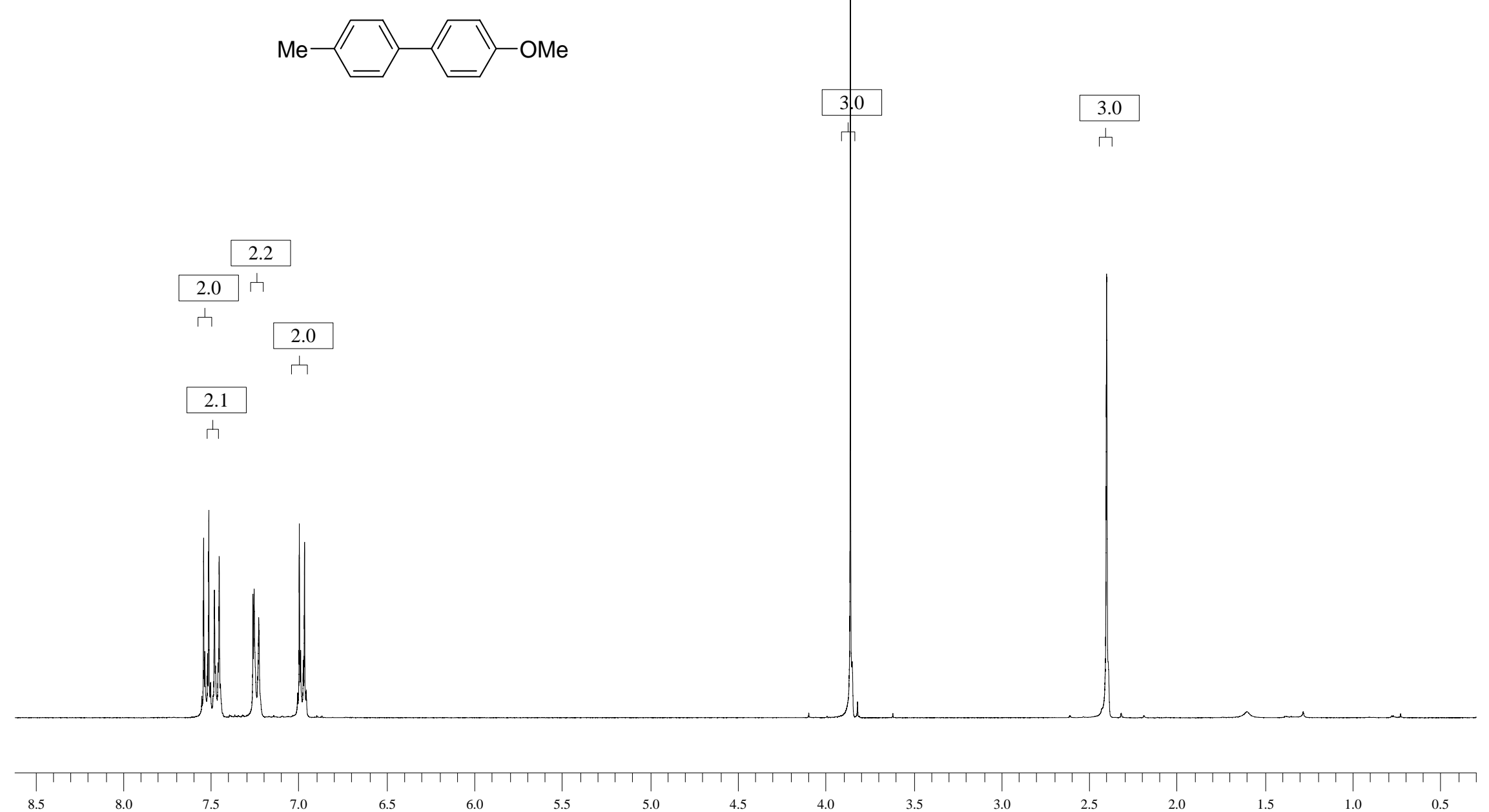

\begin{tabular}{lllllllllllll}
8.5 & 8.0 & 7.5 & 7.0 & 6.5 & 6.0 & 5.5 & 5.0 & 4.5 & 4.0 & 3.5 & 3.0 & 2.5 \\
\hline
\end{tabular} 


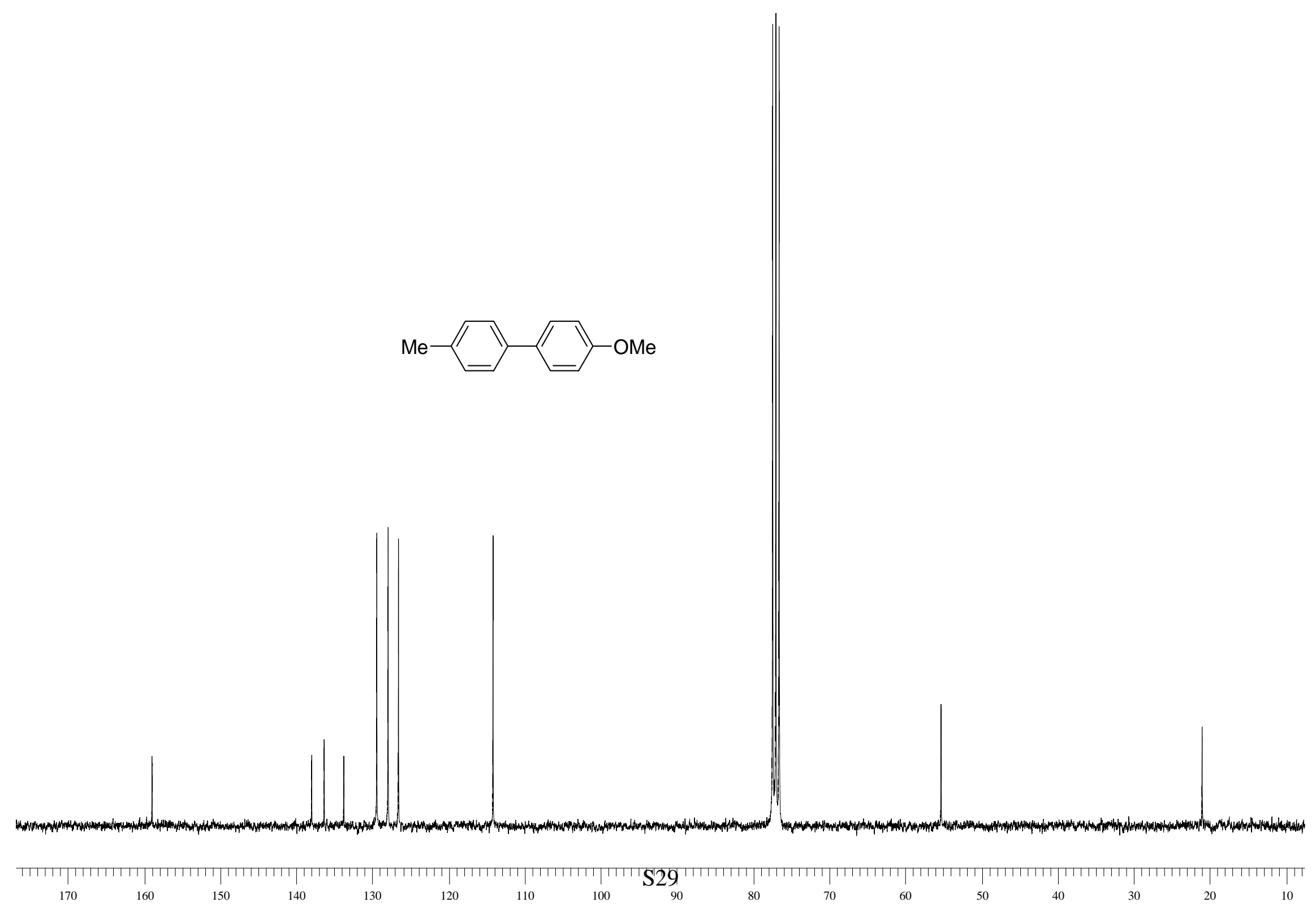



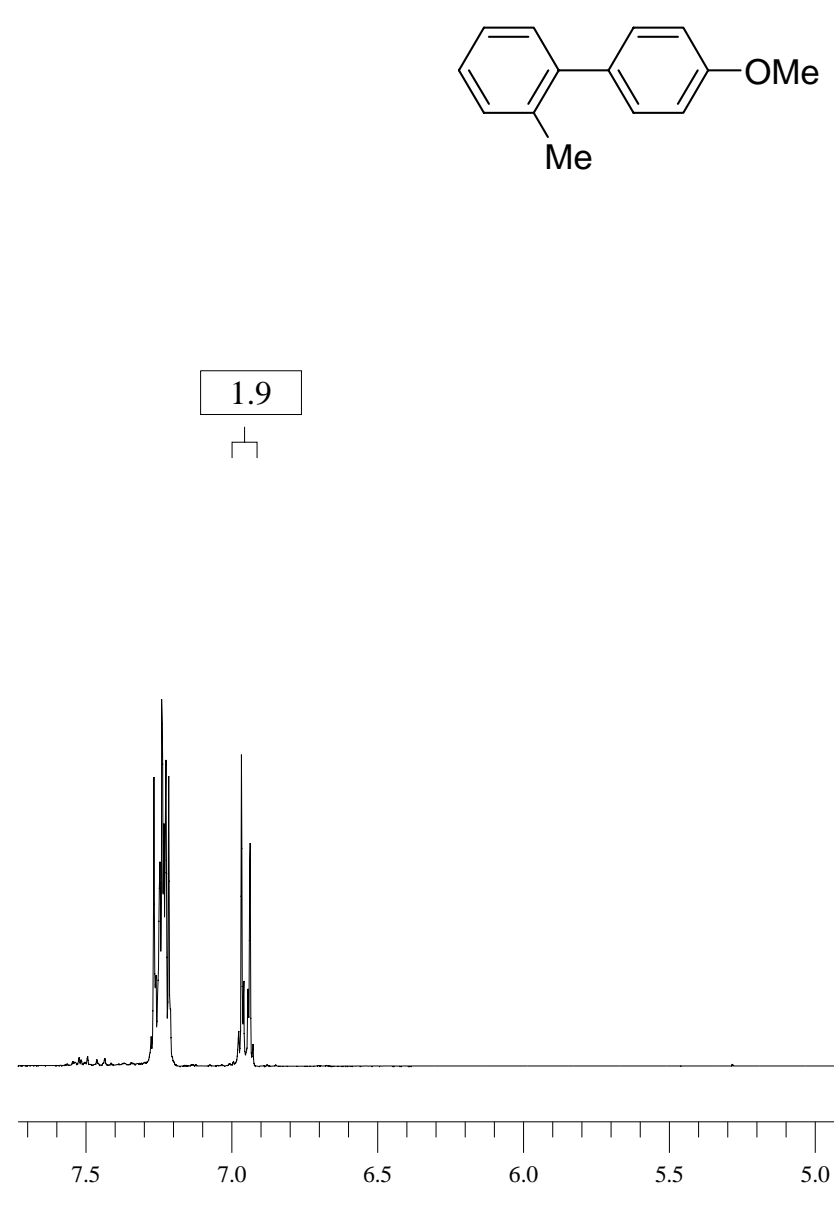


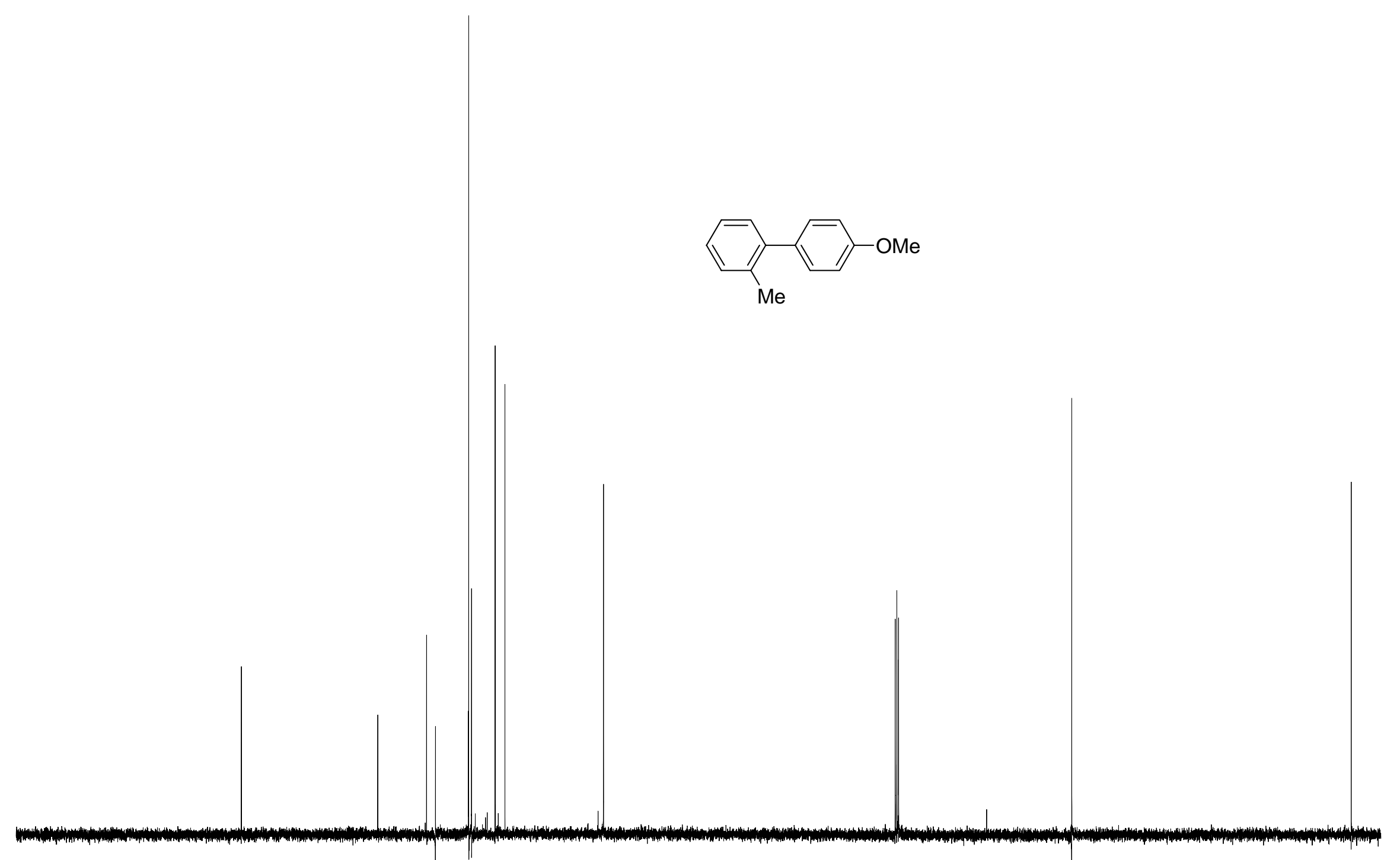

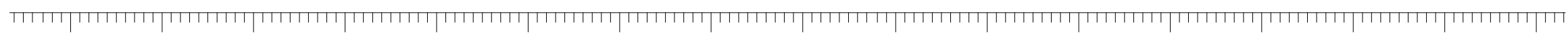
130
120
90
80
$70 \quad 60$
$50 \quad 40$

$30 \quad 20$ 


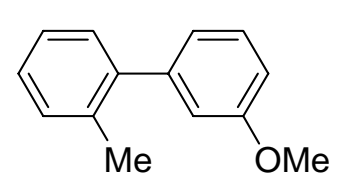

5.0

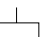

2.8
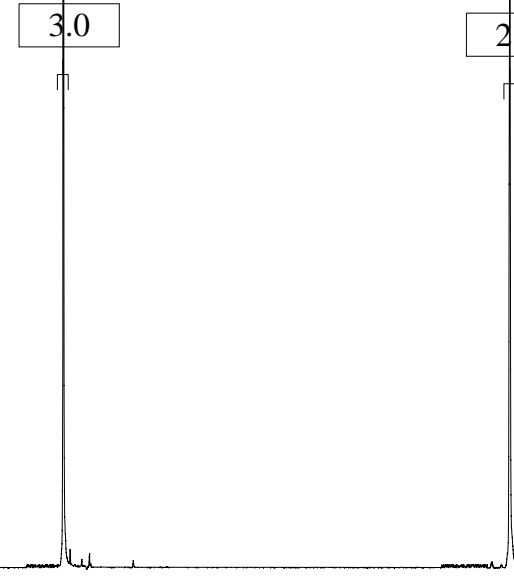

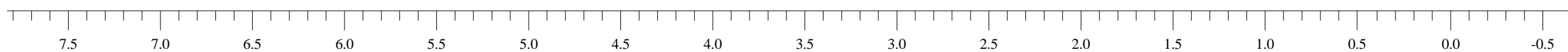




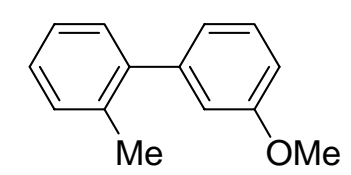

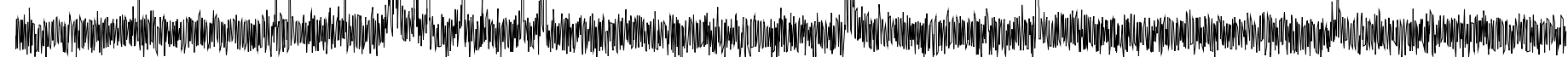

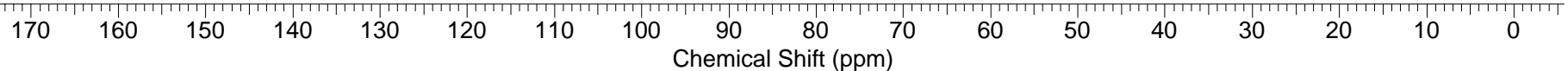



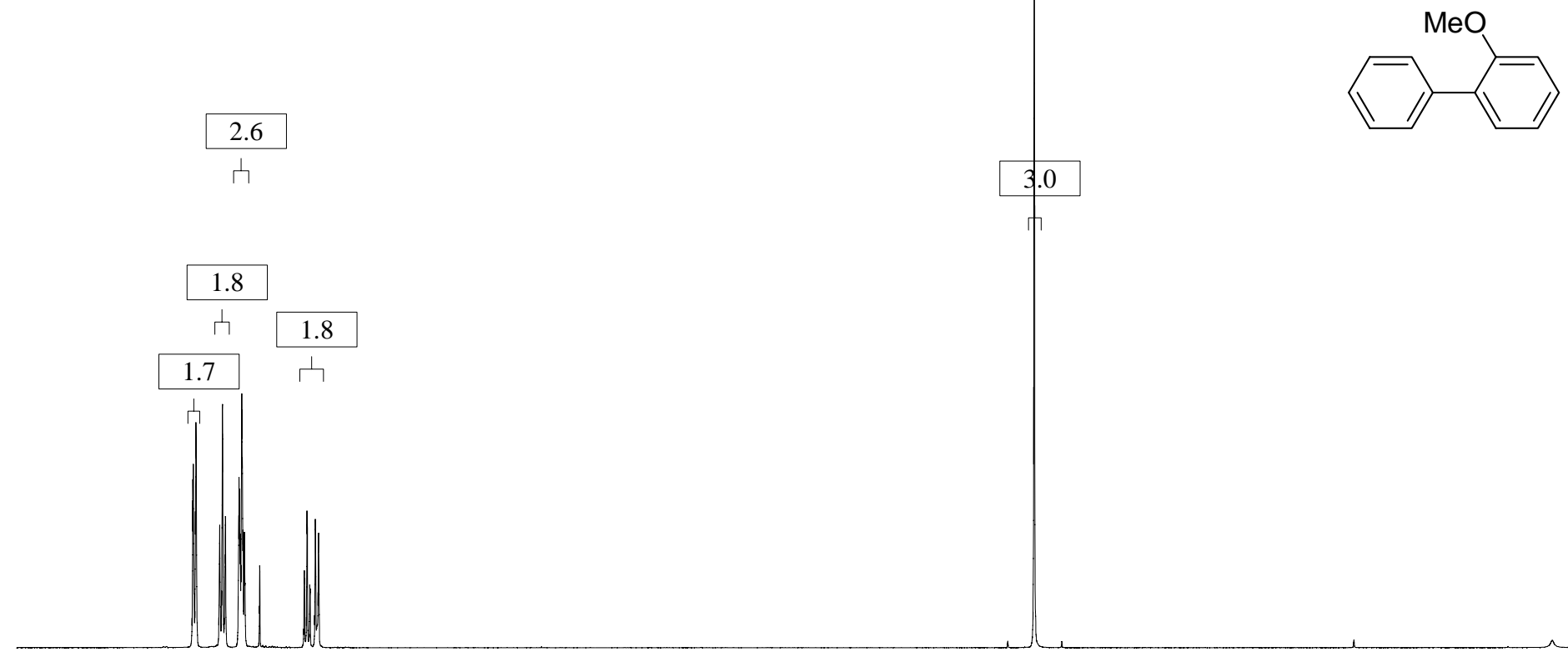

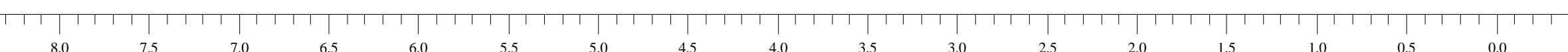




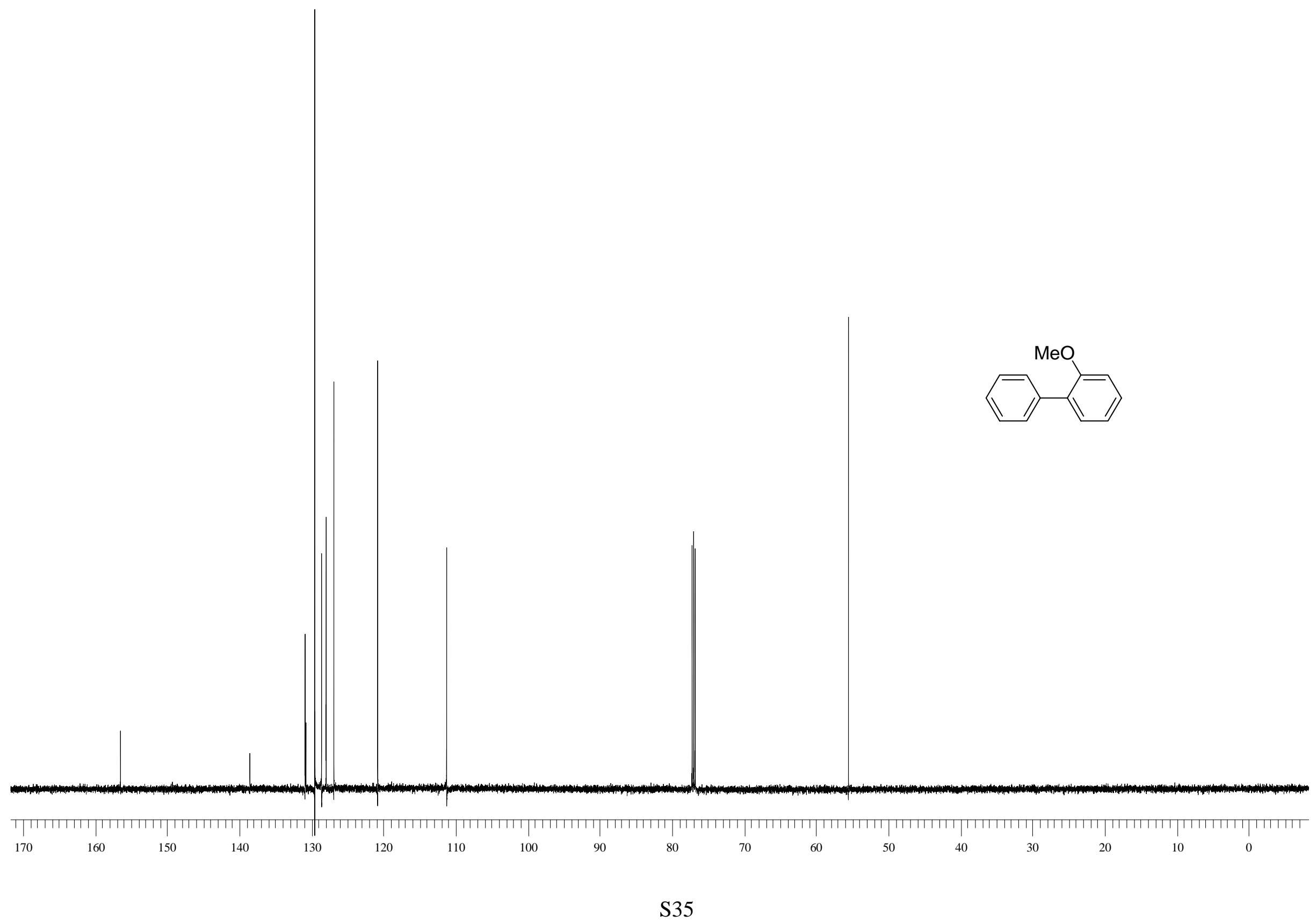




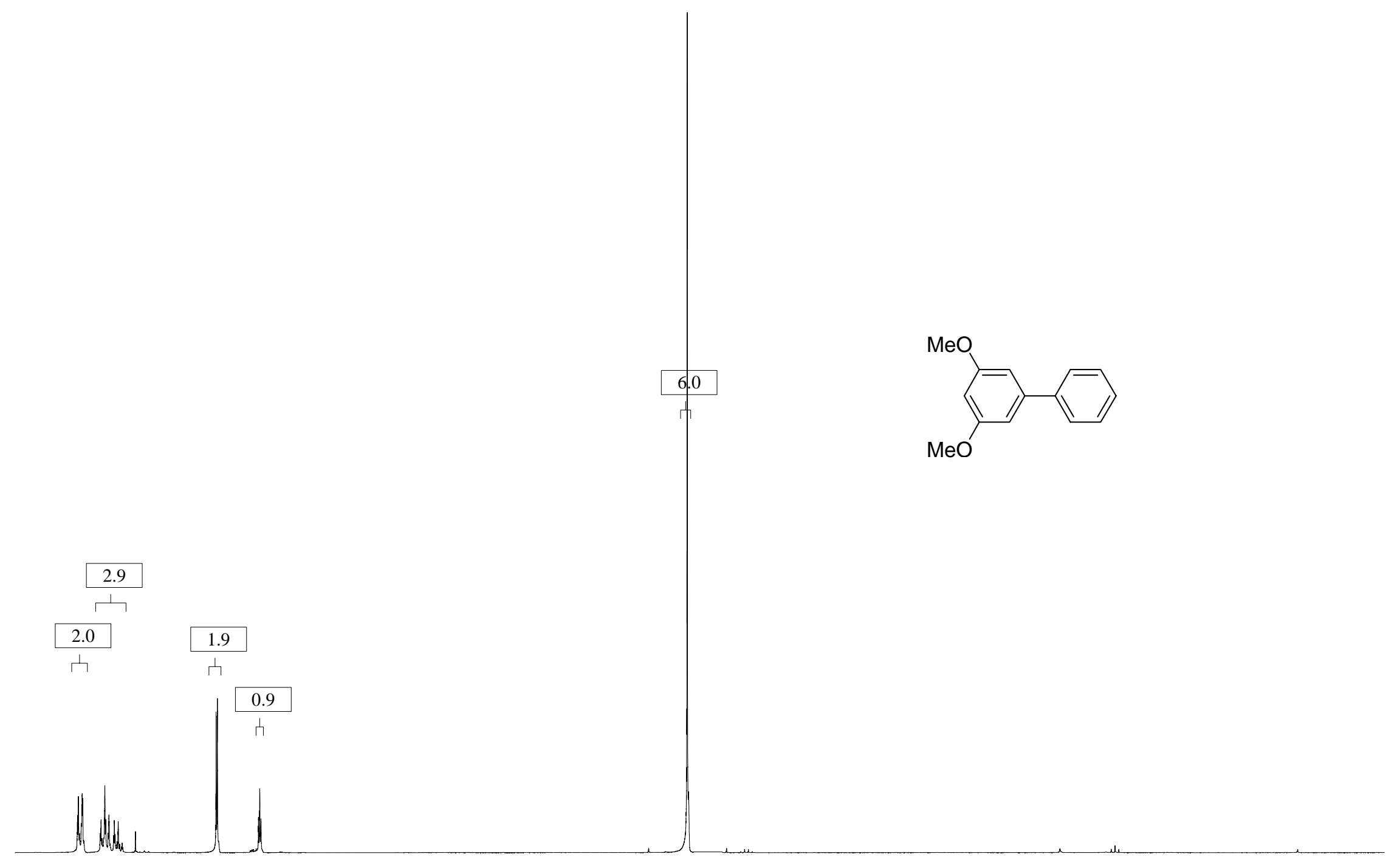

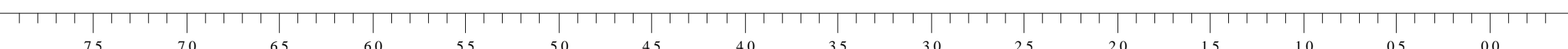




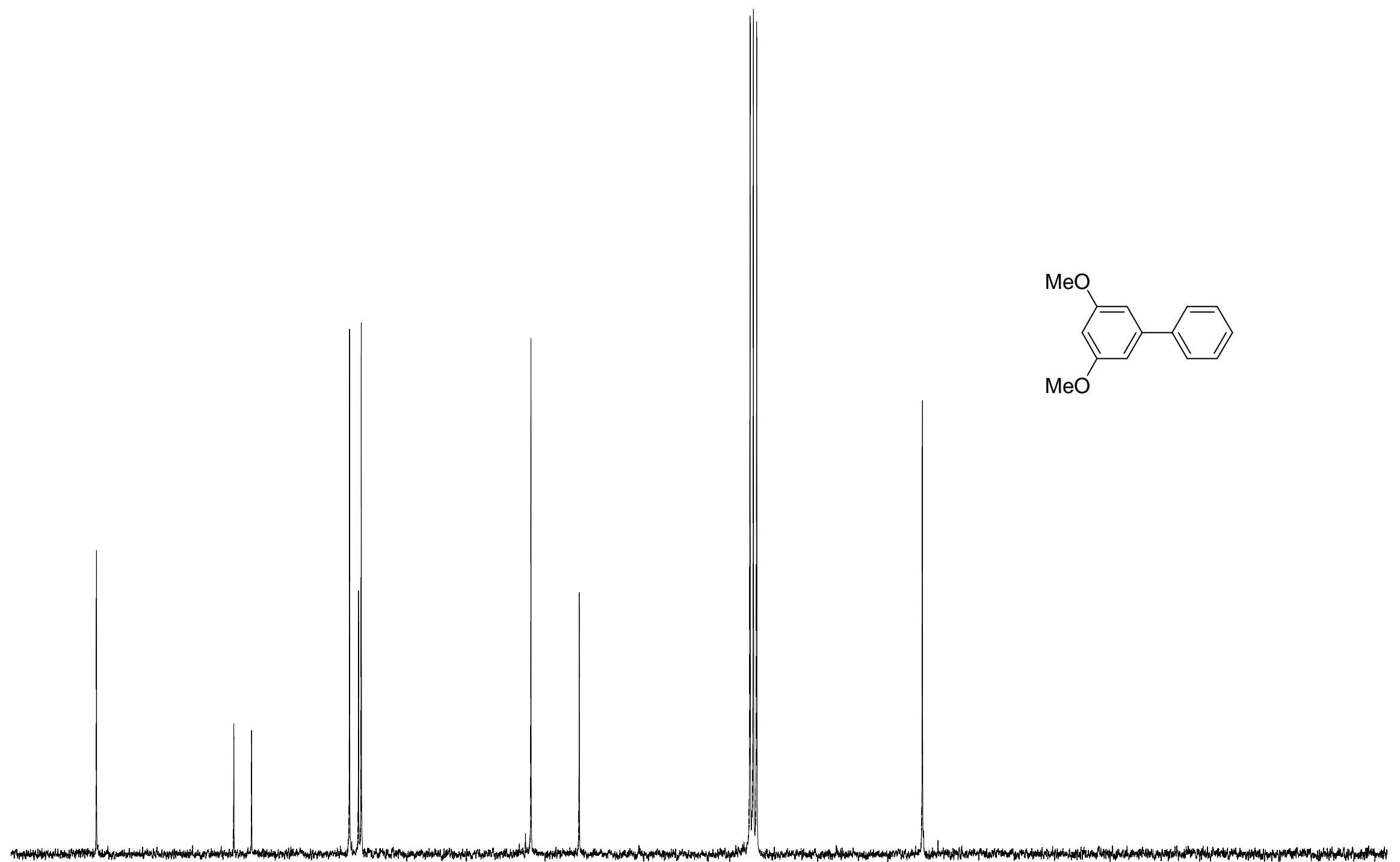

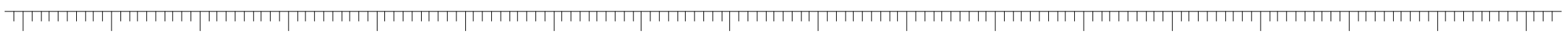




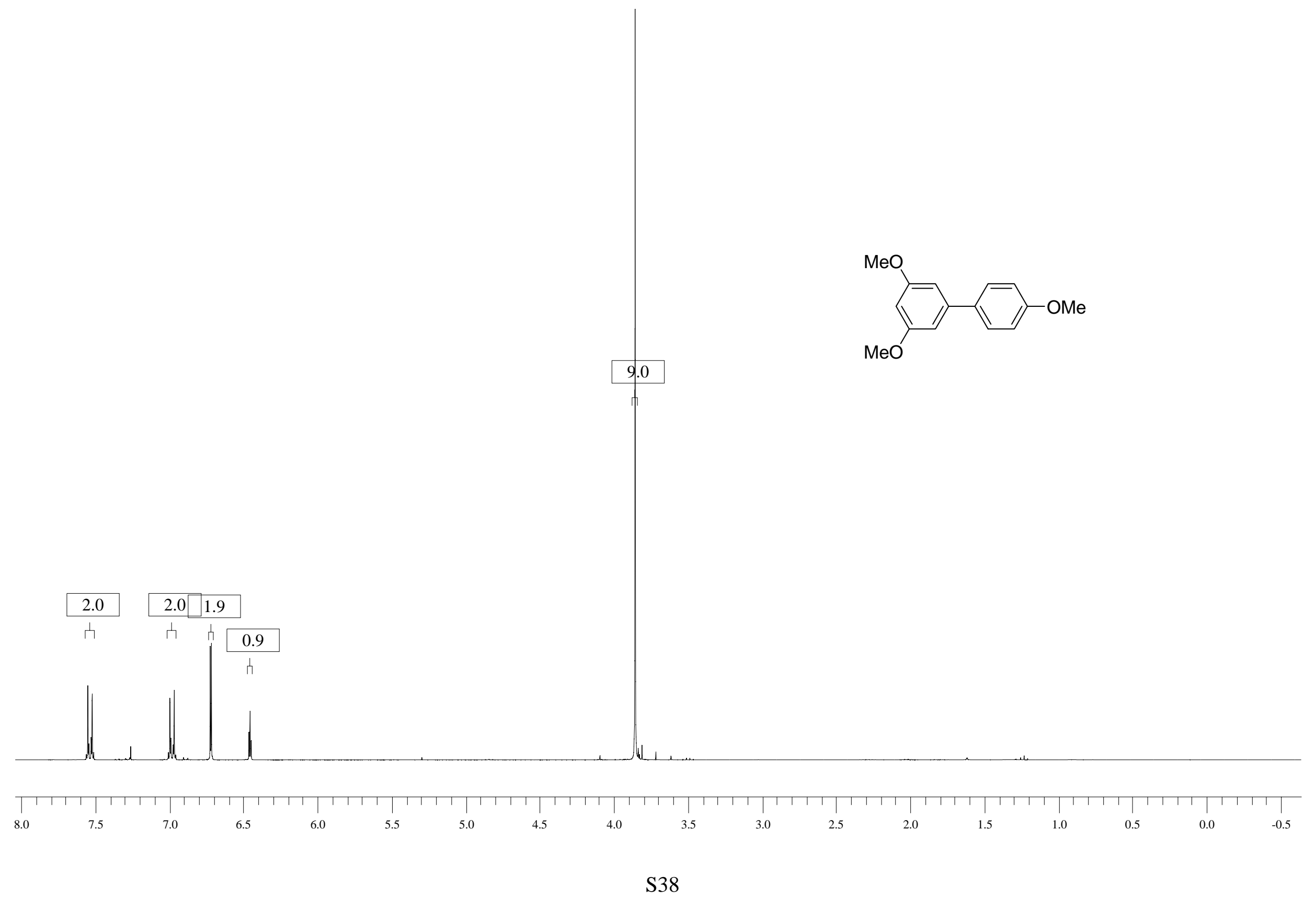




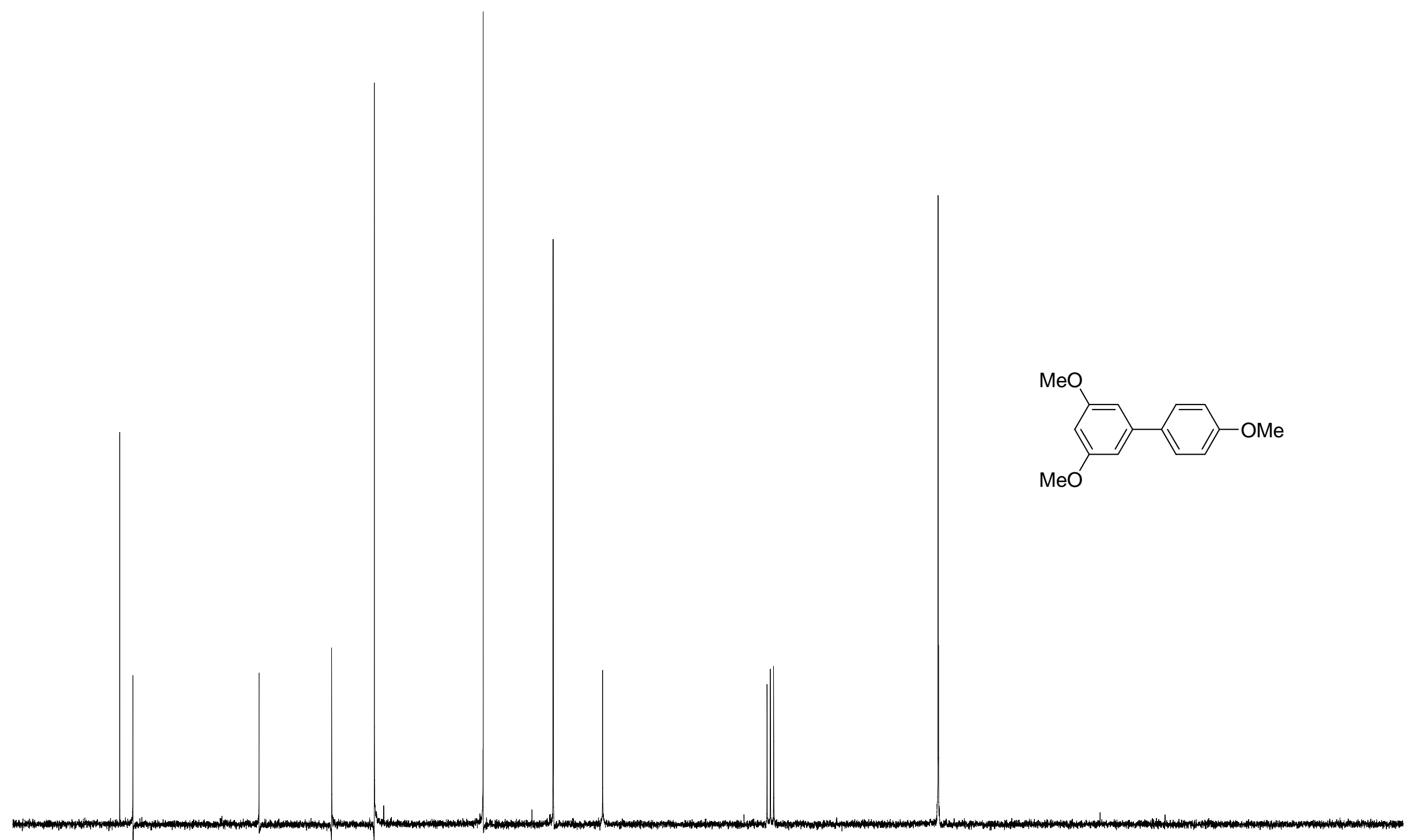

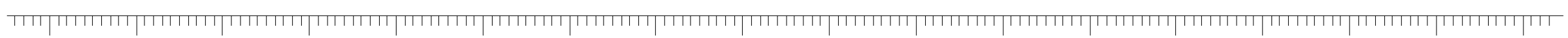




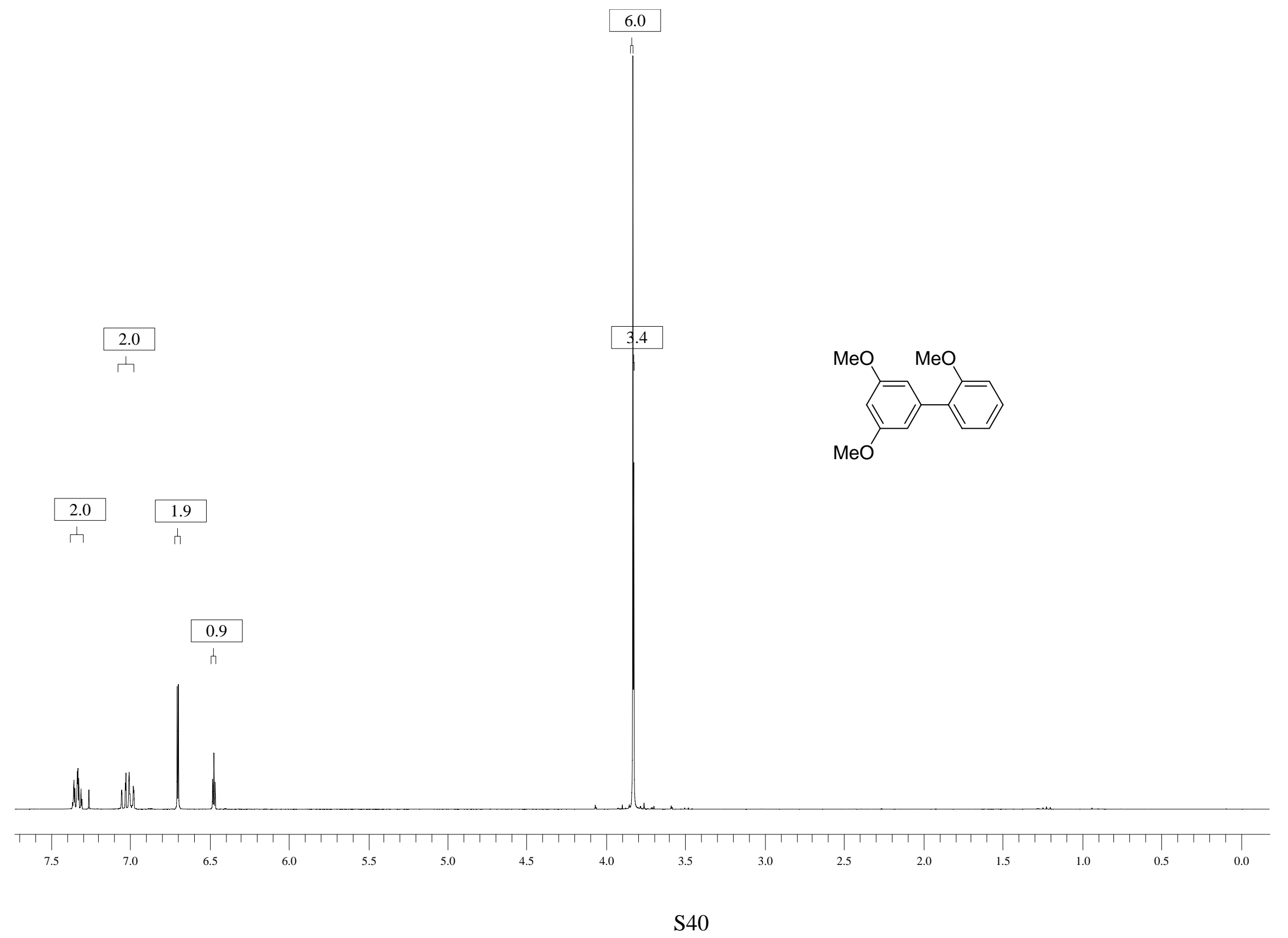




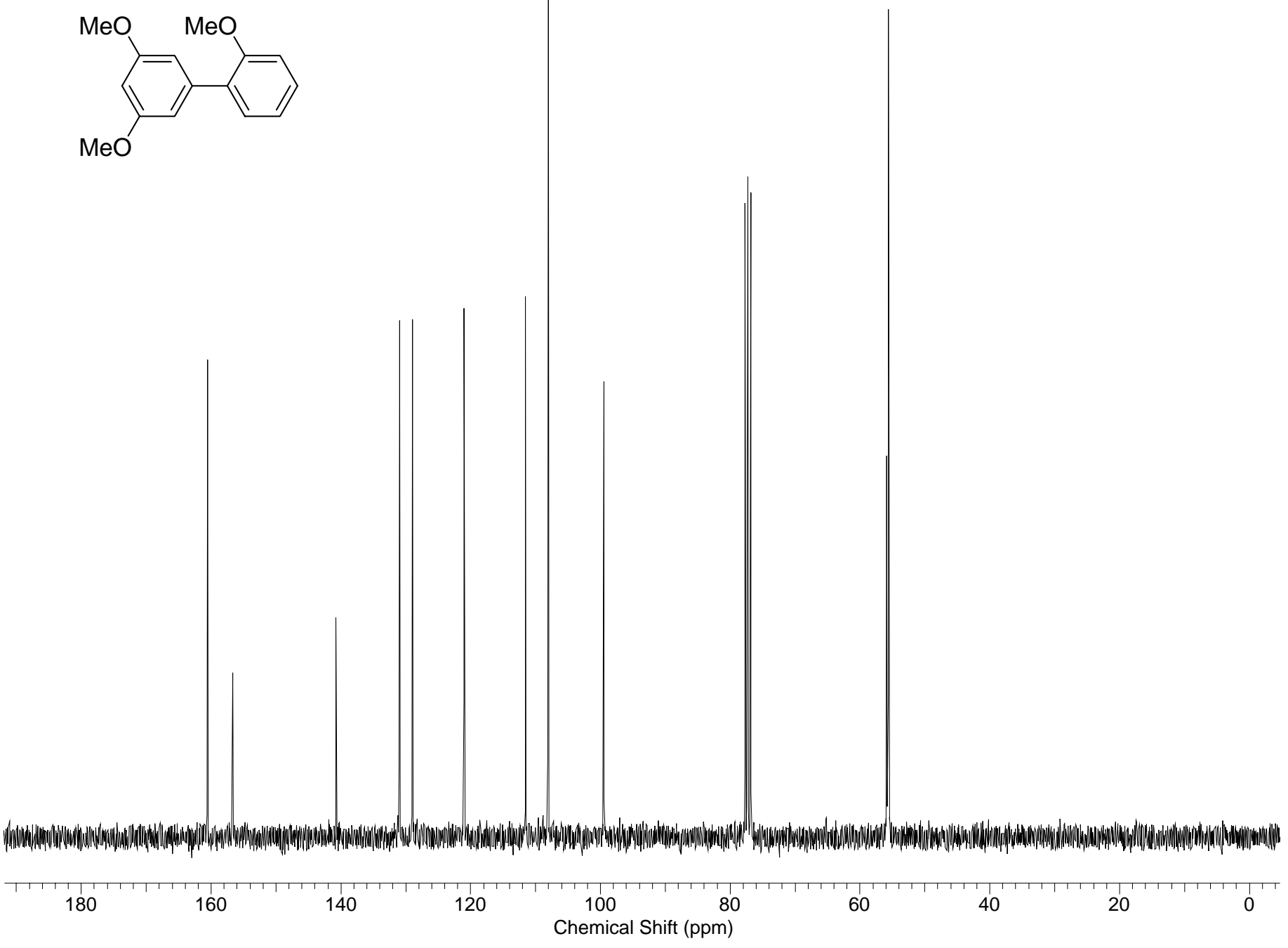




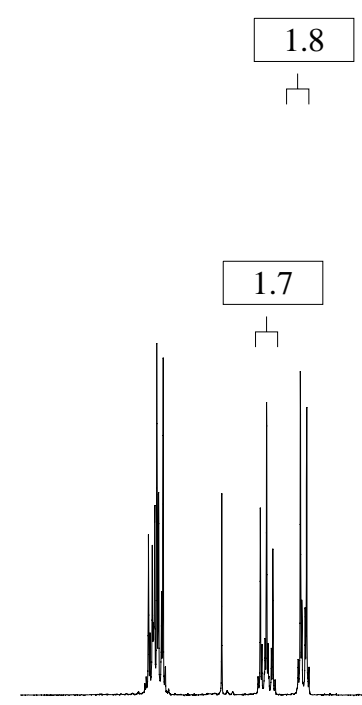




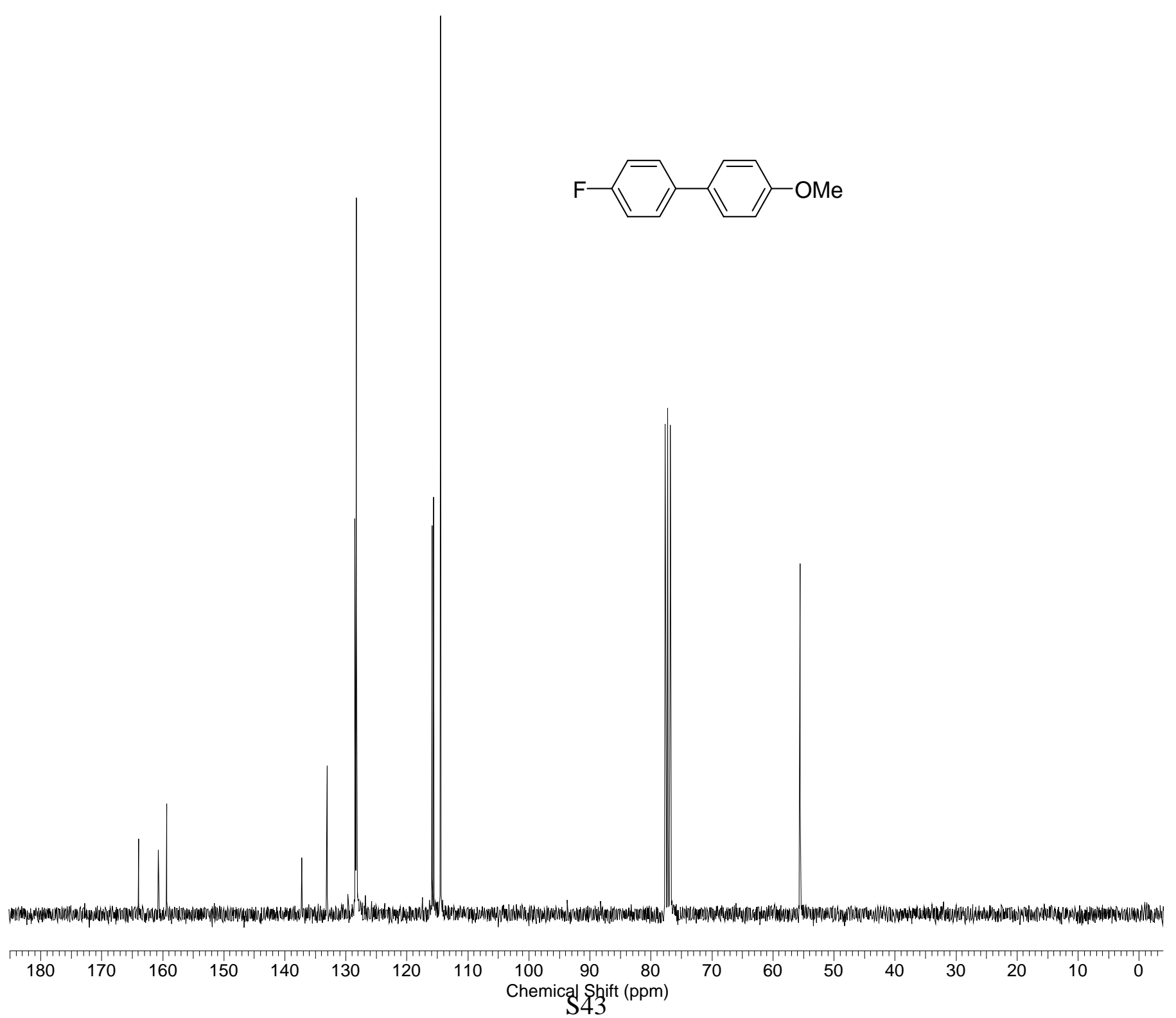




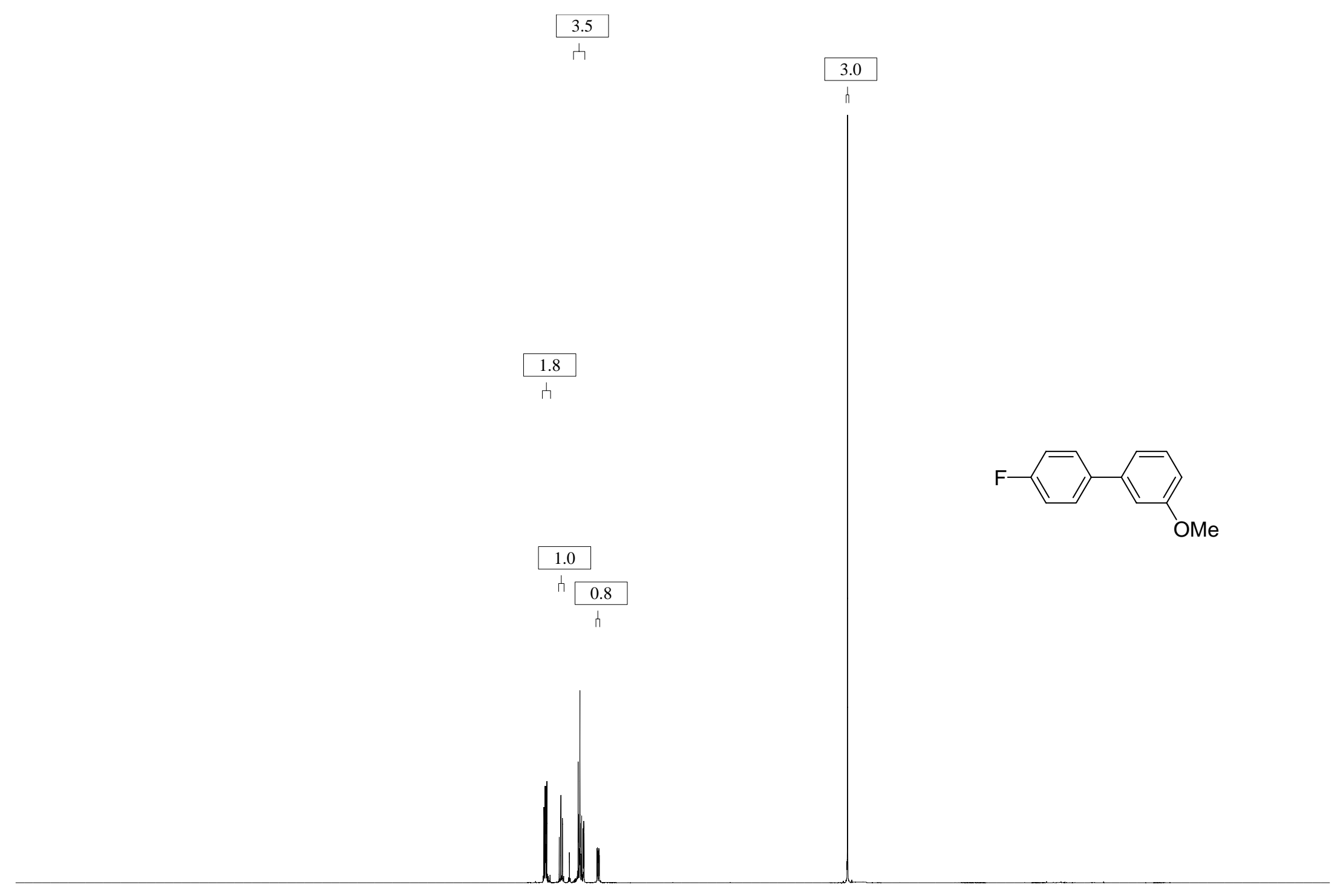

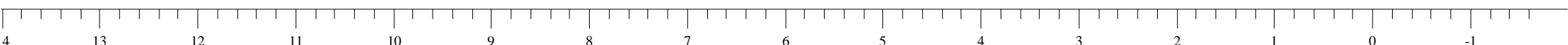




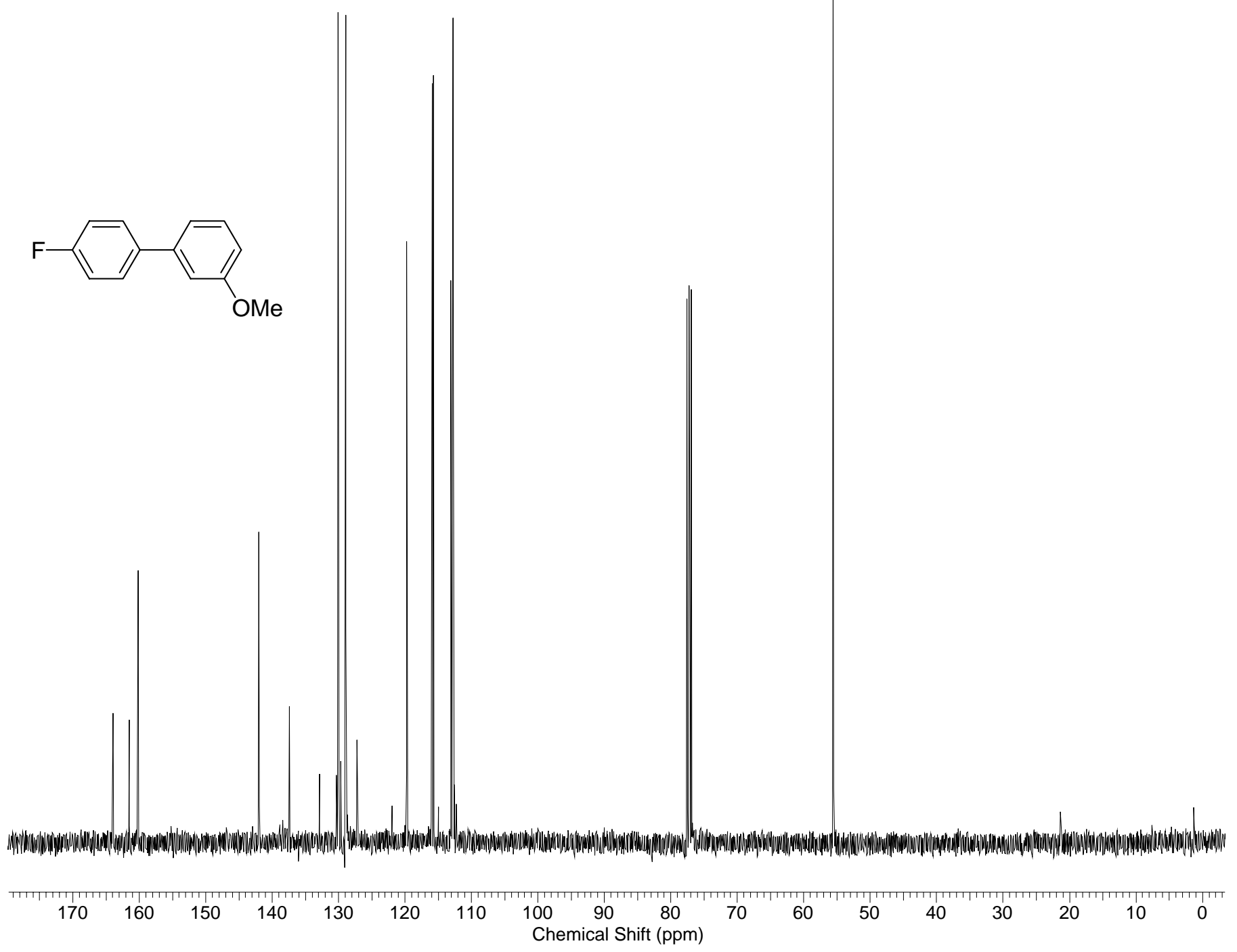



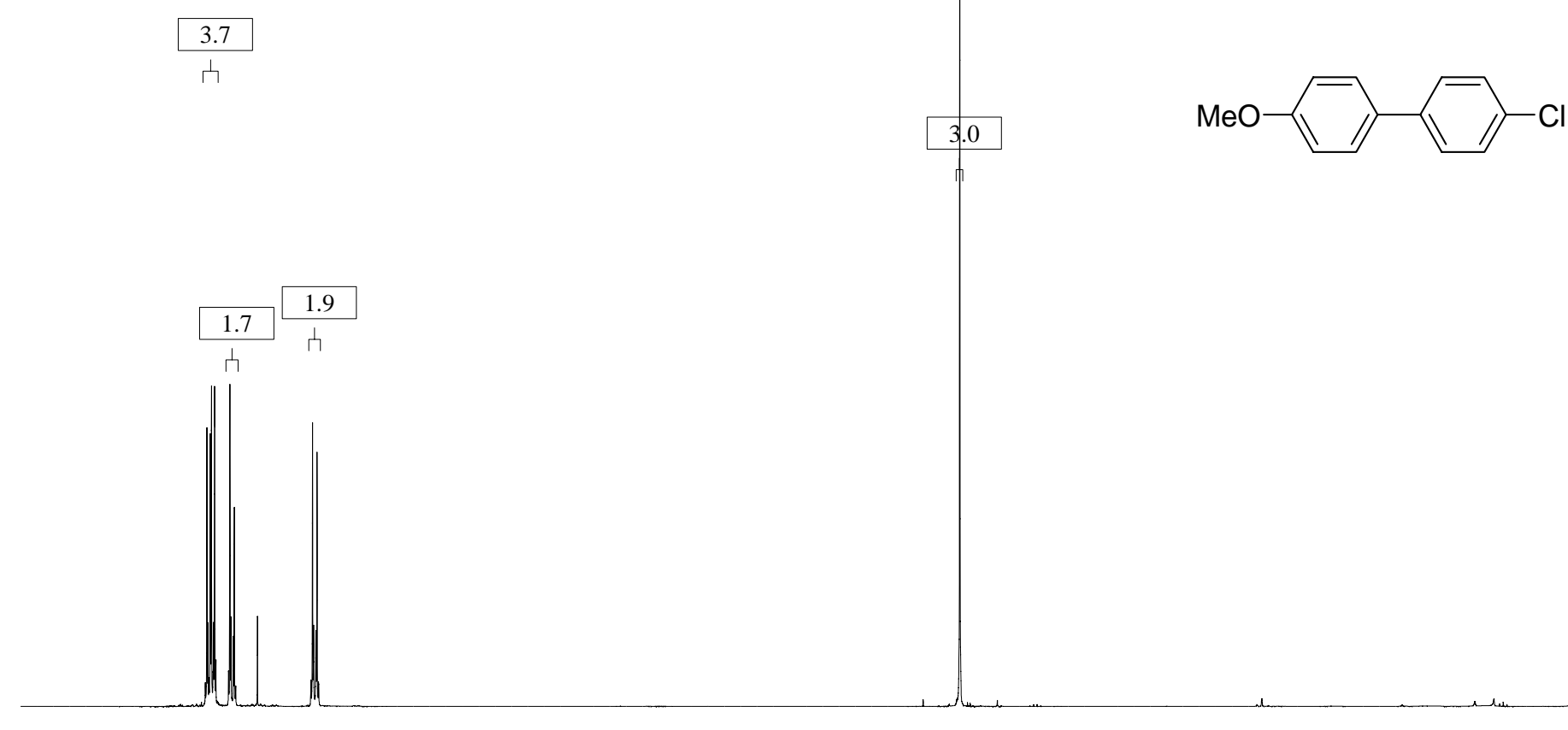

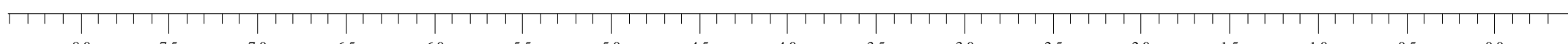




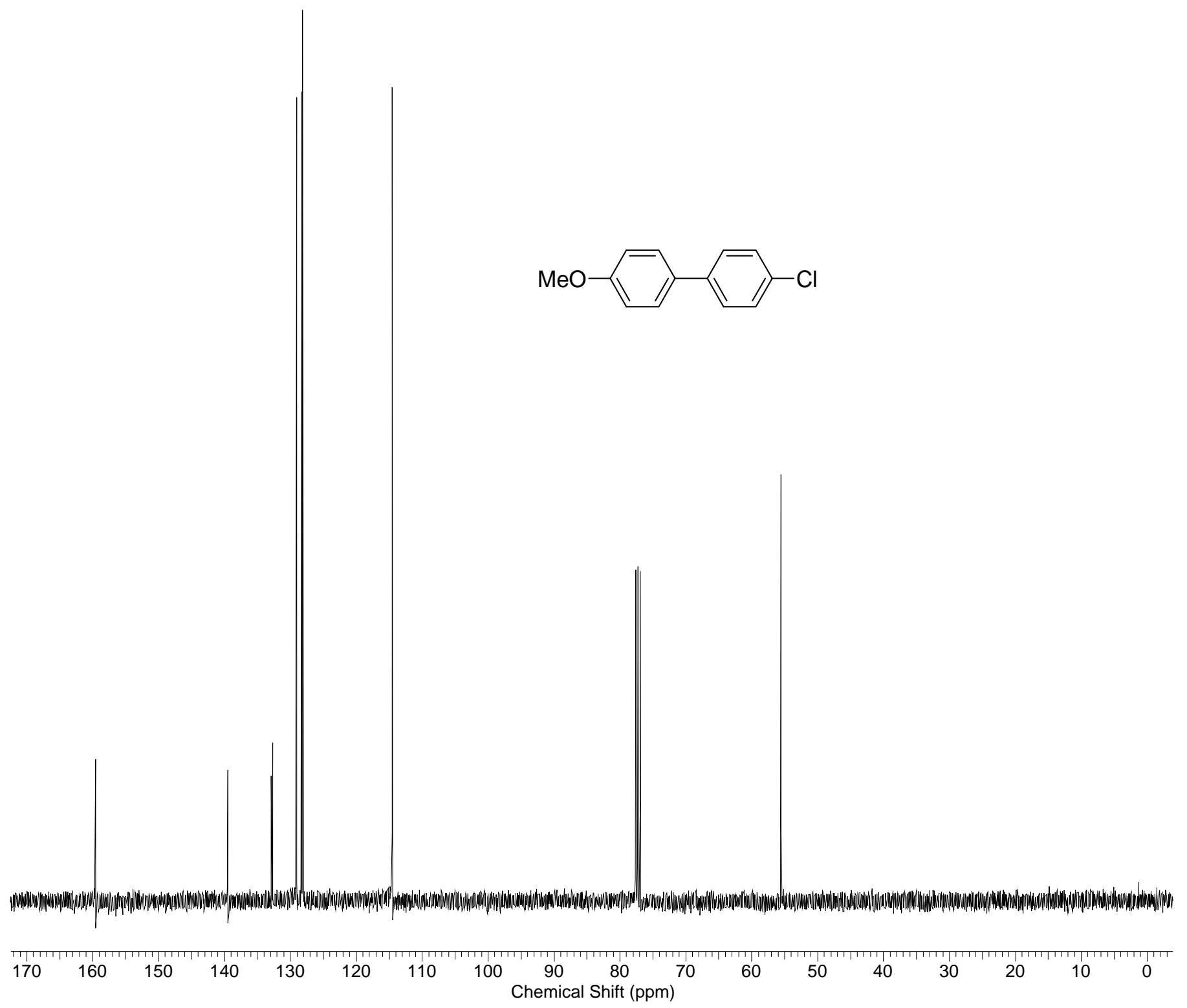


\begin{tabular}{|l|l|}
\hline 1.9 & 1.9 \\
\hline \multicolumn{1}{|l}{} & \multicolumn{1}{|l}{} \\
\hline
\end{tabular}

0.9

\begin{tabular}{|l|}
\hline 0.9 \\
\hline 1
\end{tabular}

0.9

0.9
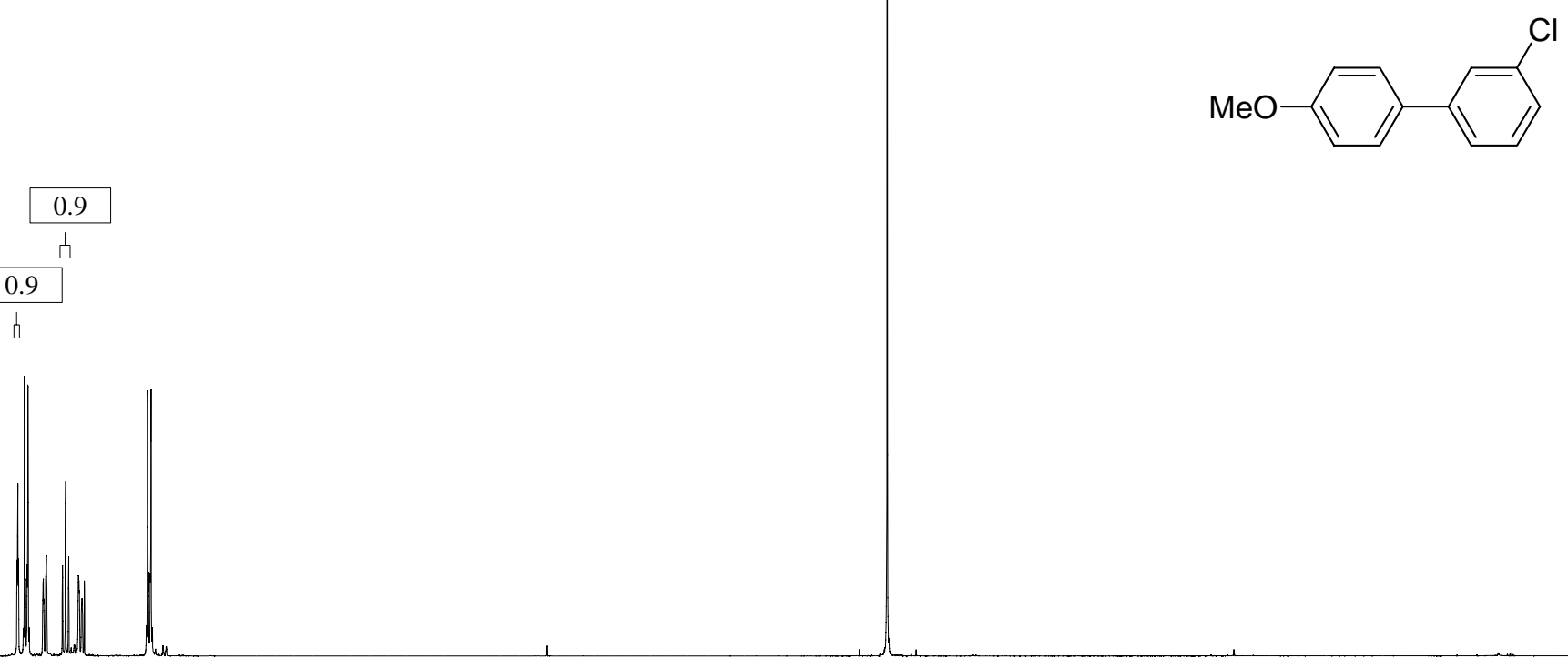


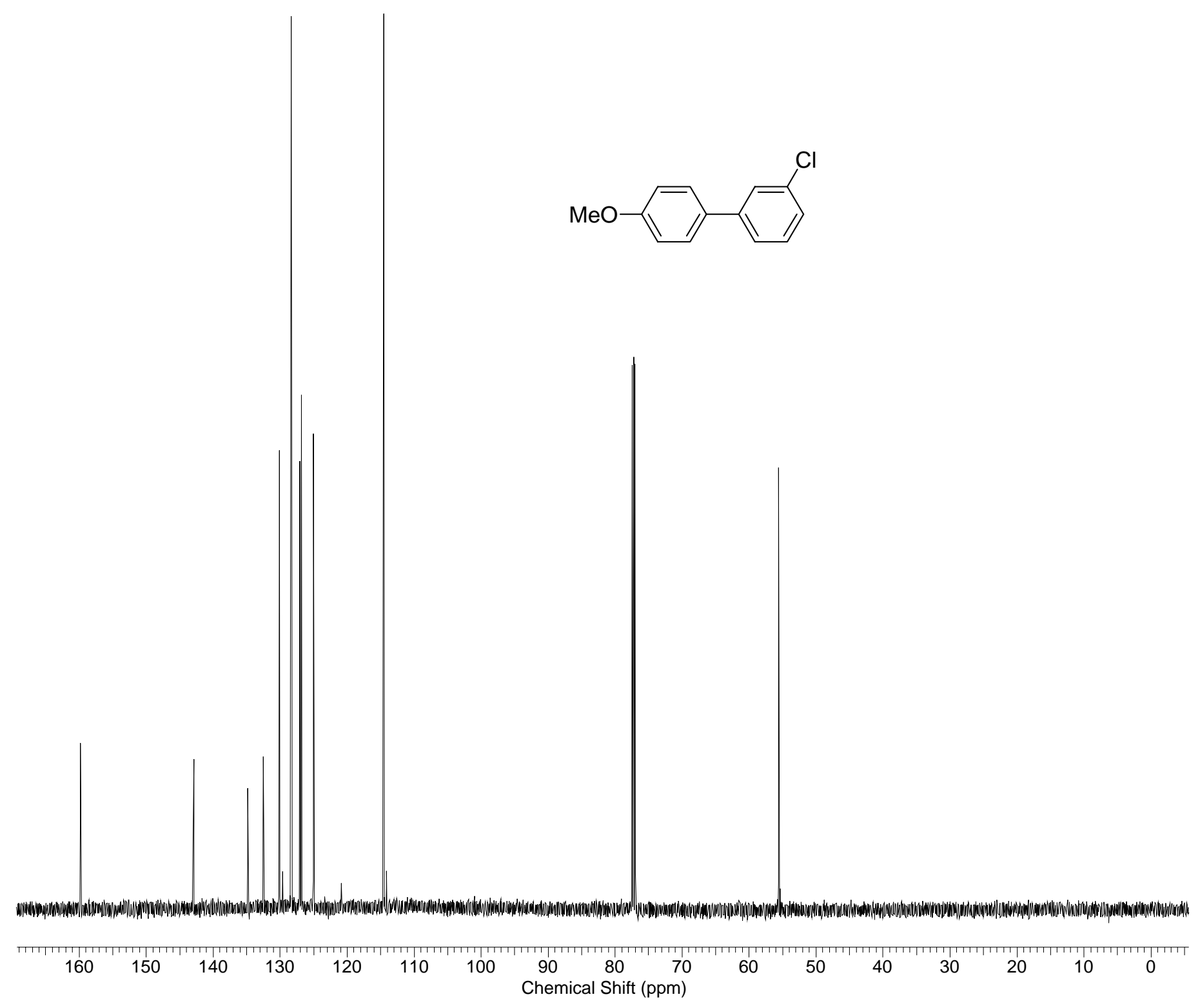




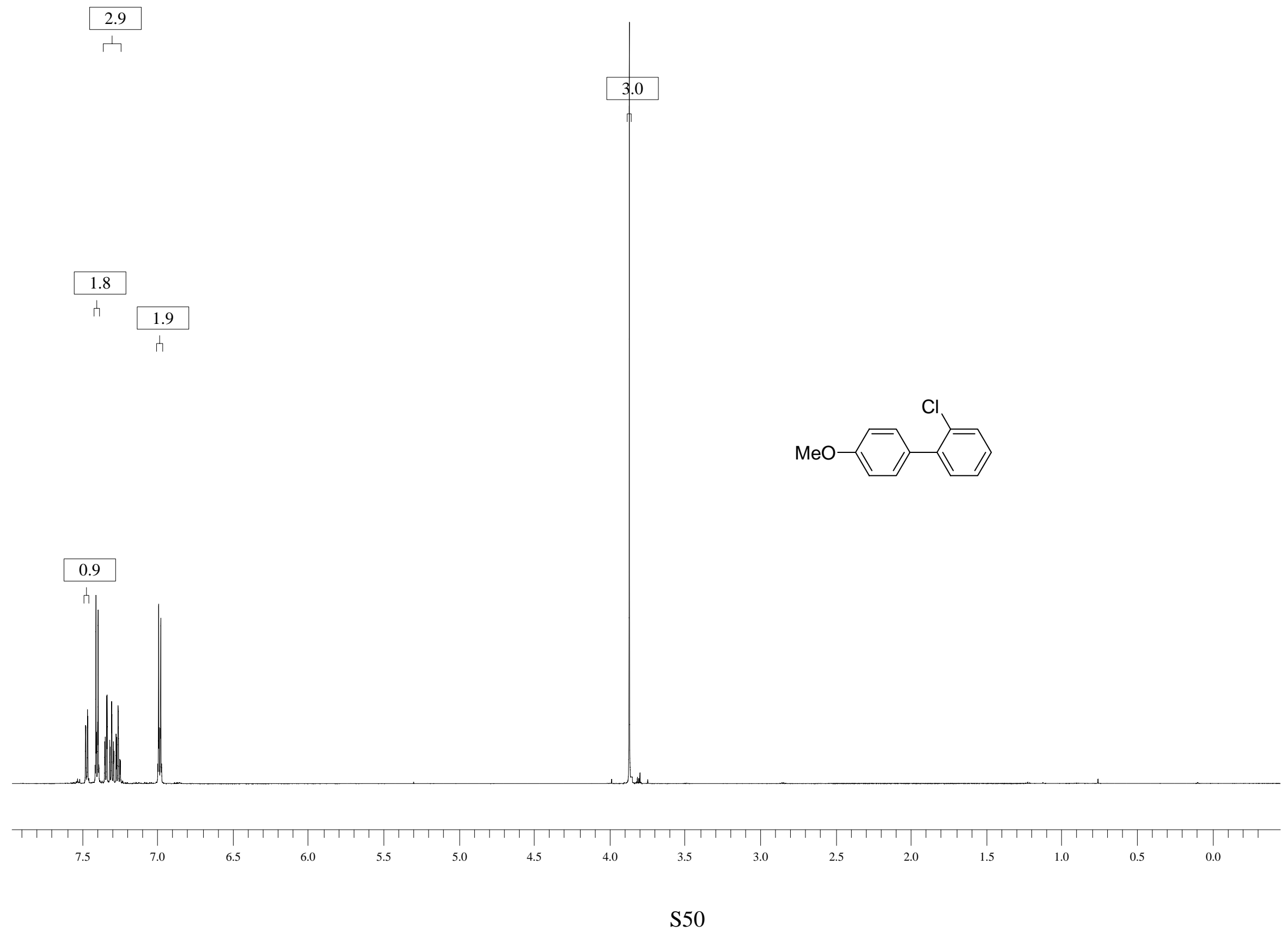




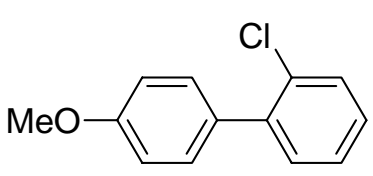

111111111111111111111 



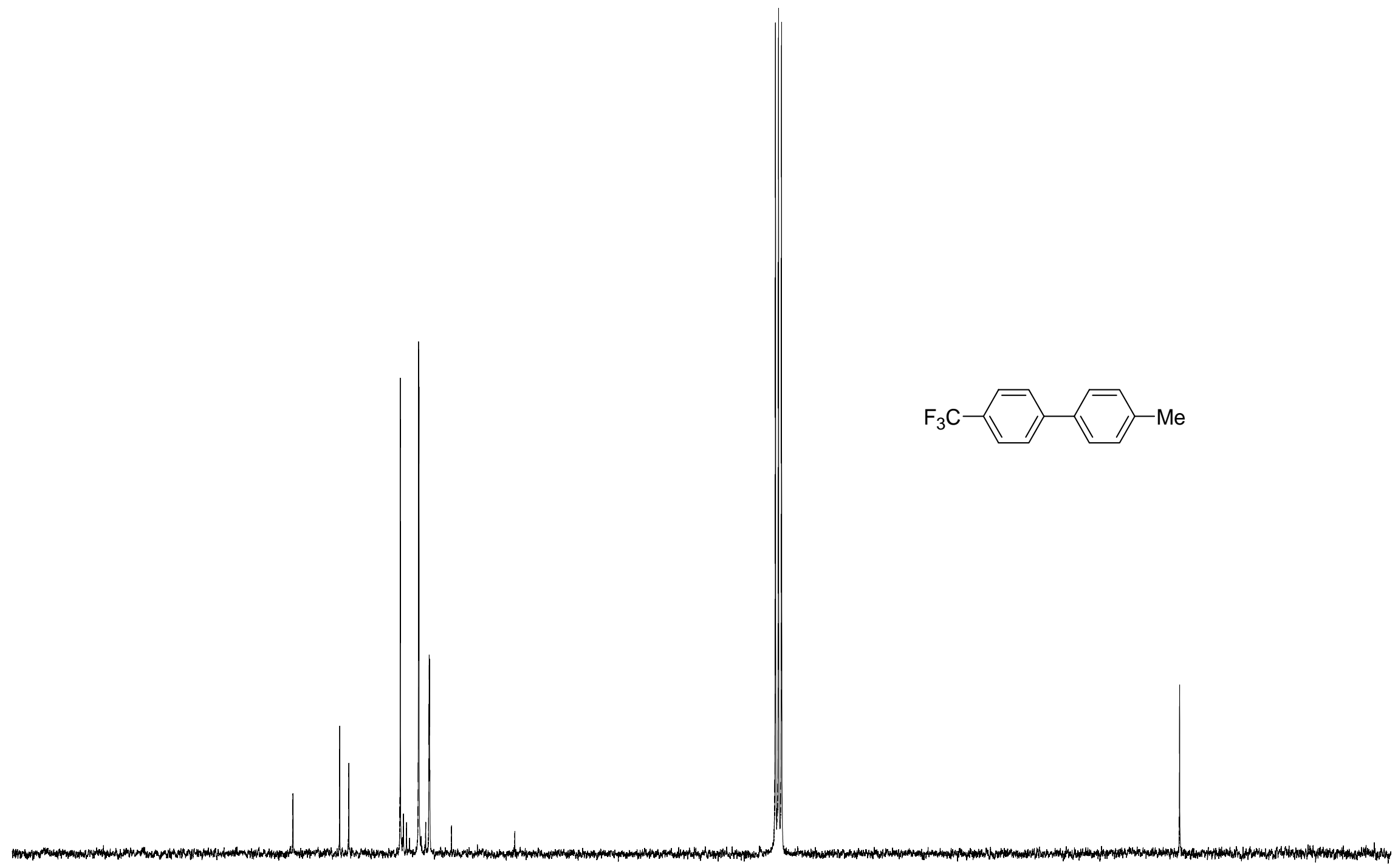

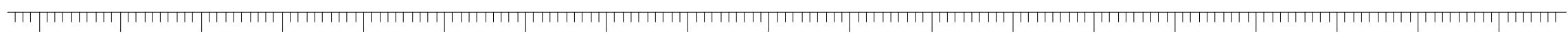

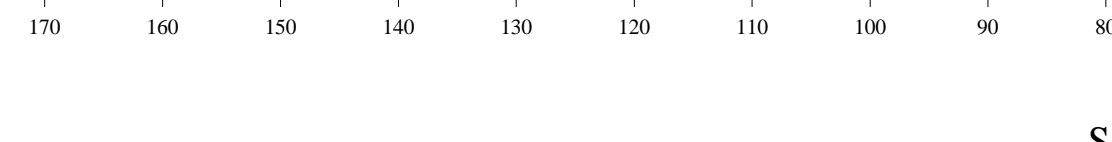




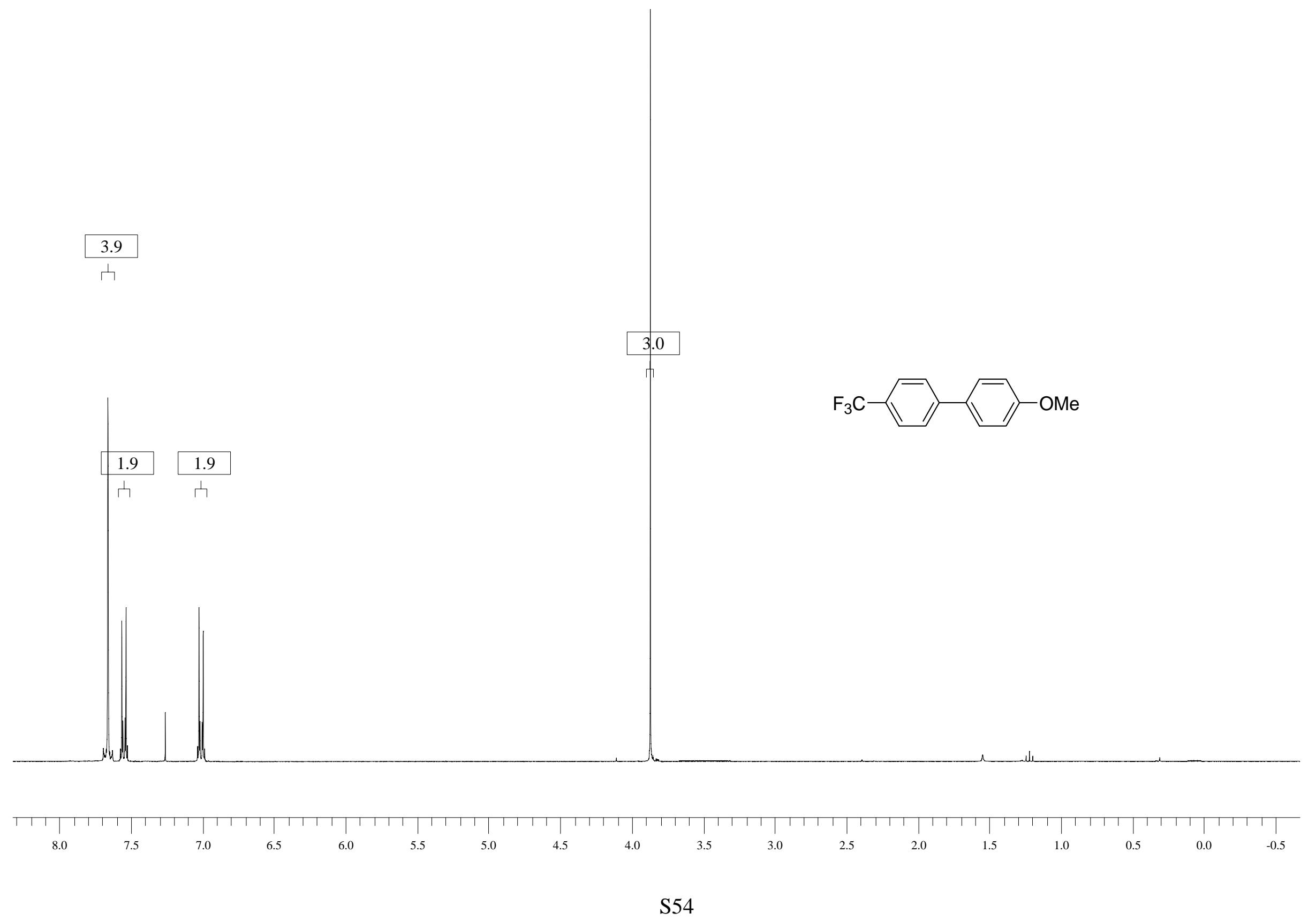




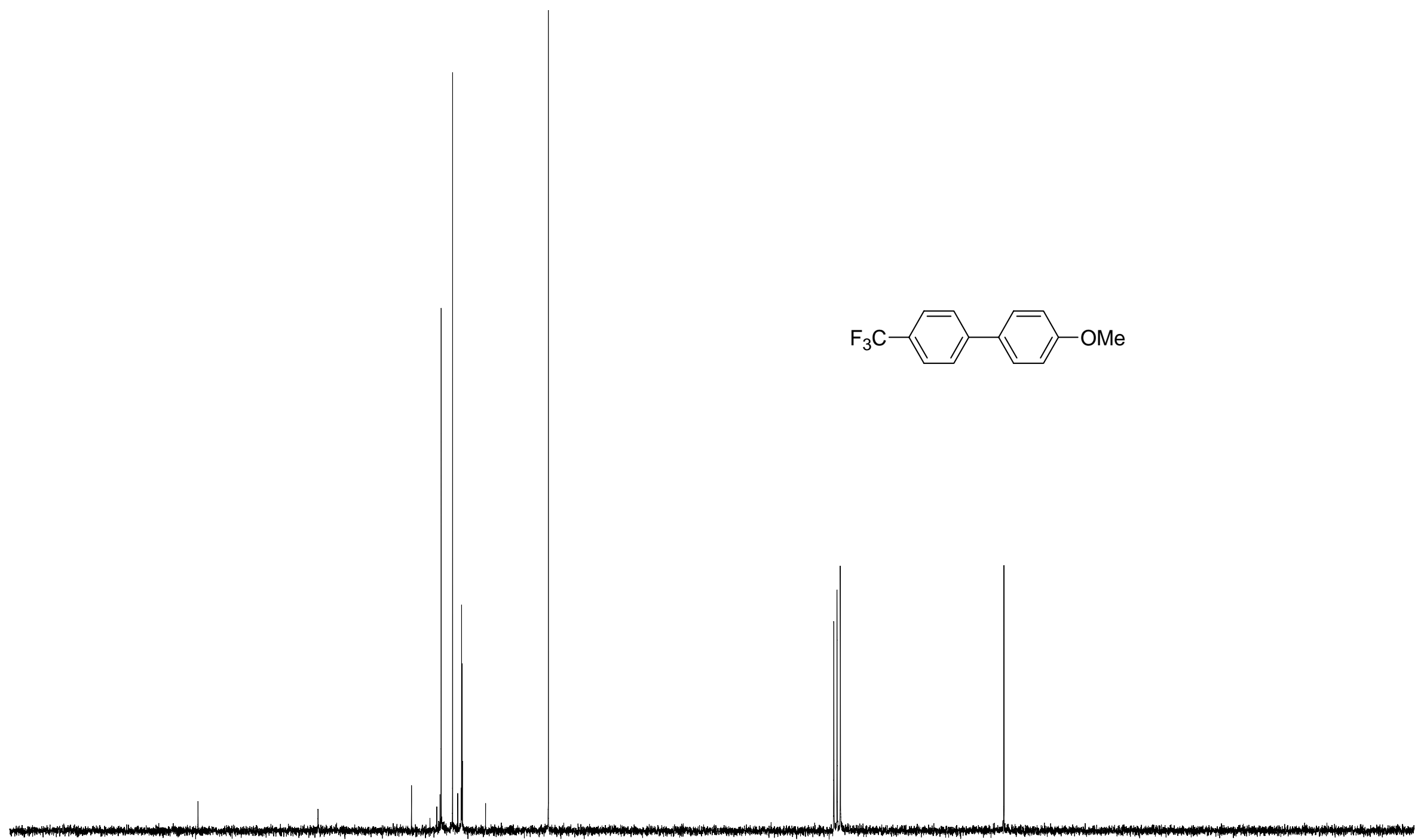

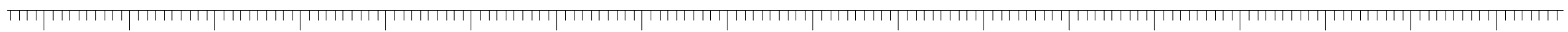

$\begin{array}{lllllllll}170 & 160 & 150 & 140 & 130 & 120 & 110 & 100 & 90\end{array}$




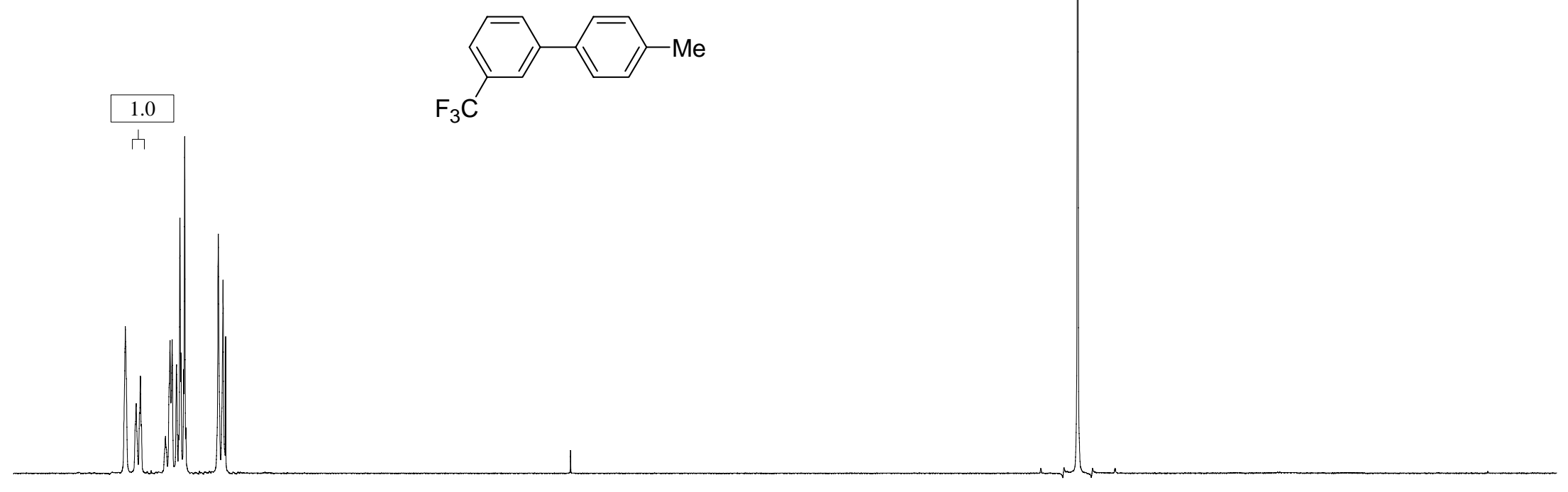




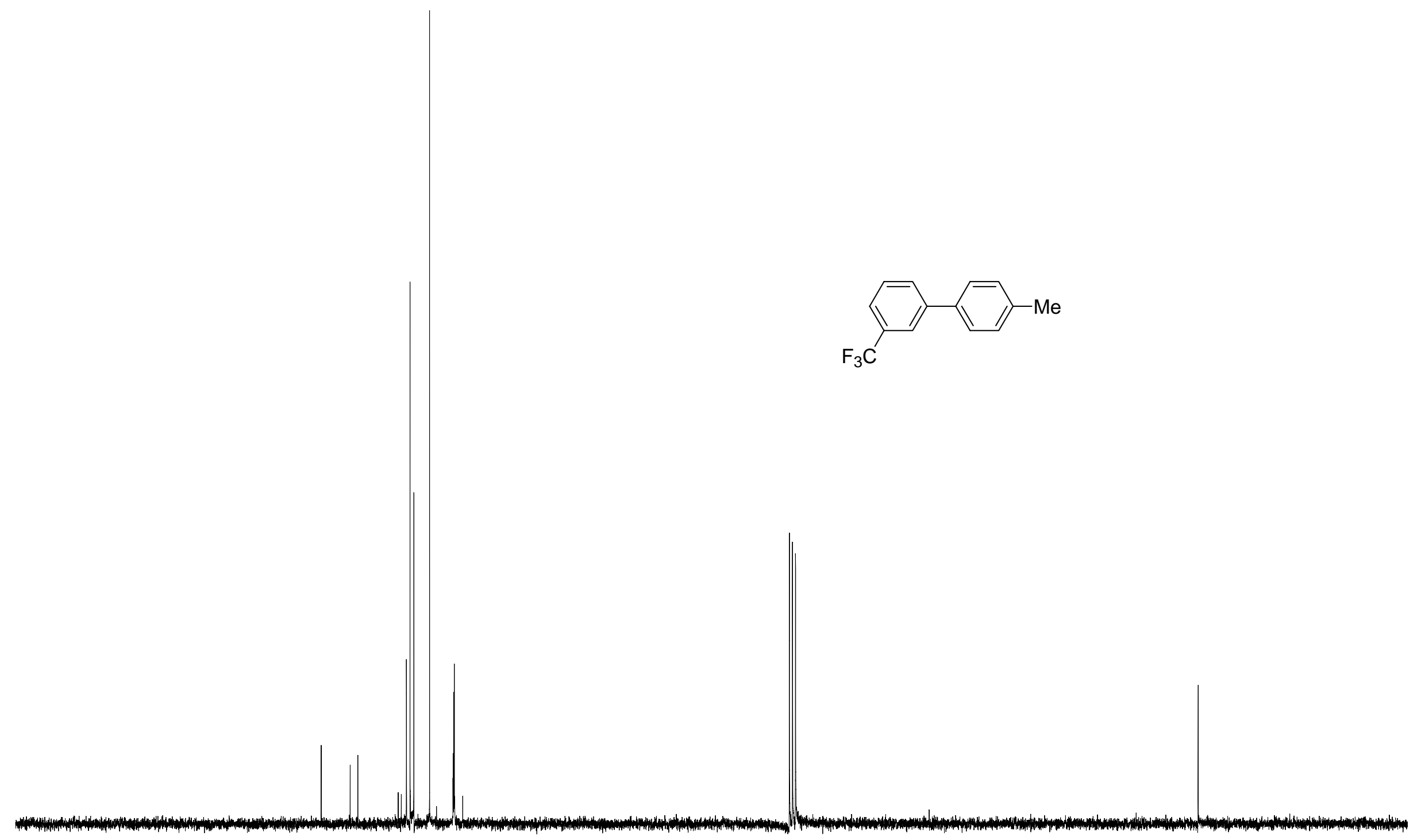

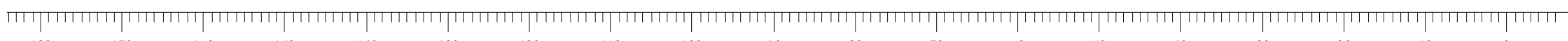

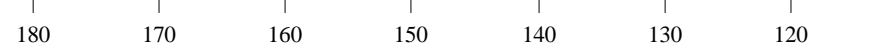




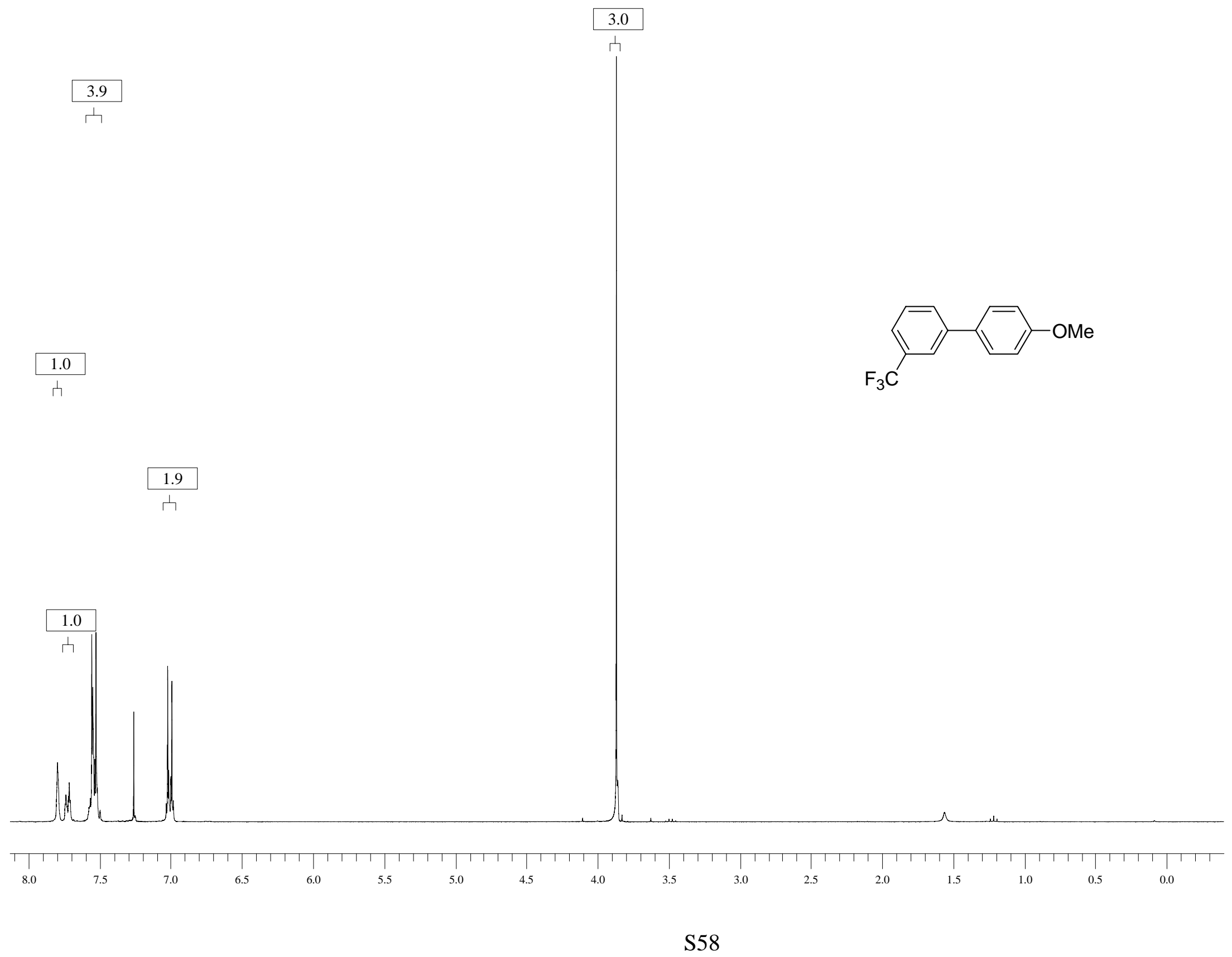




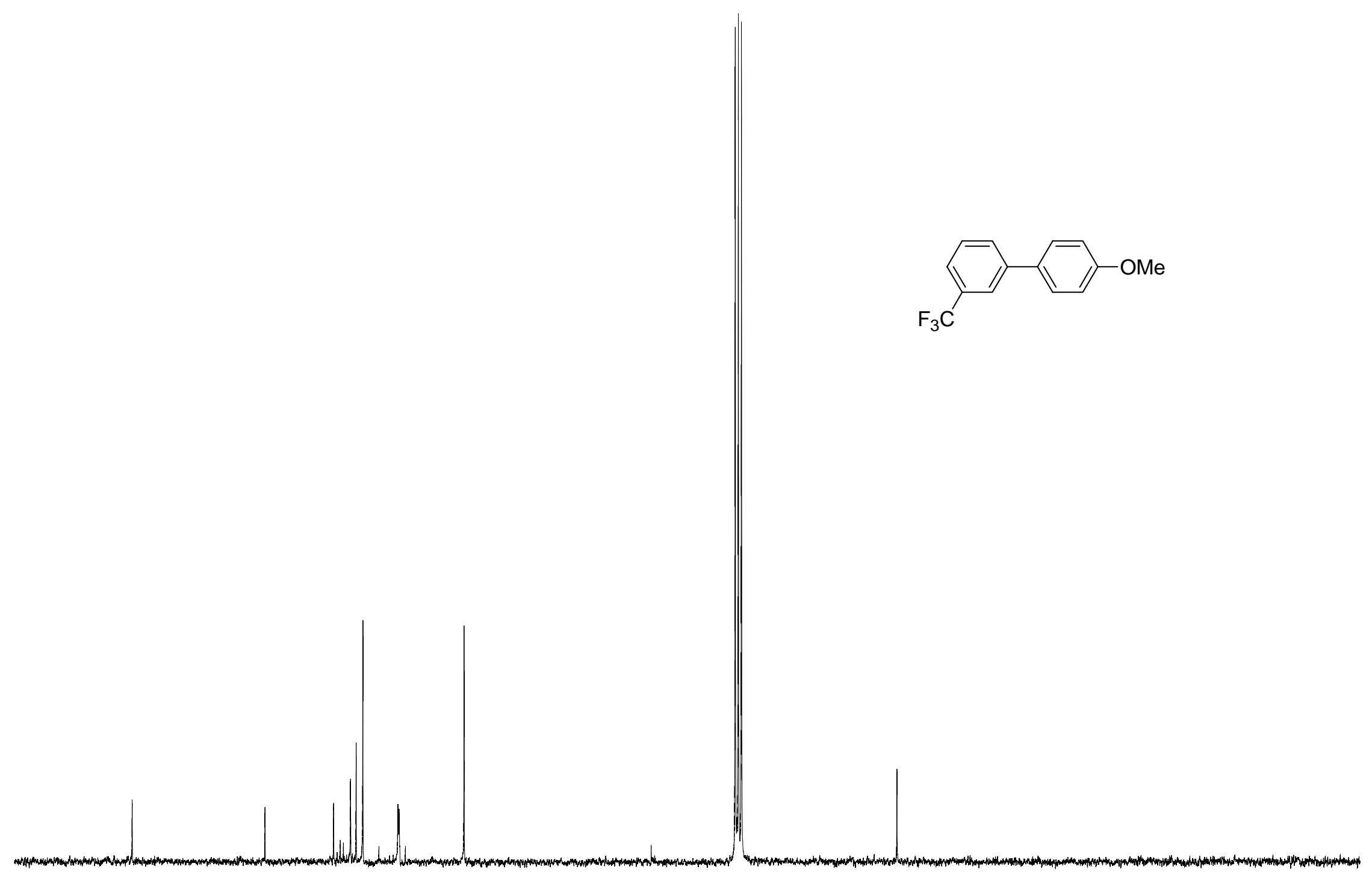

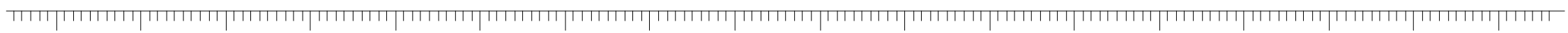
140
30
120
$10 \quad 100$
$90 \quad 80$

60 


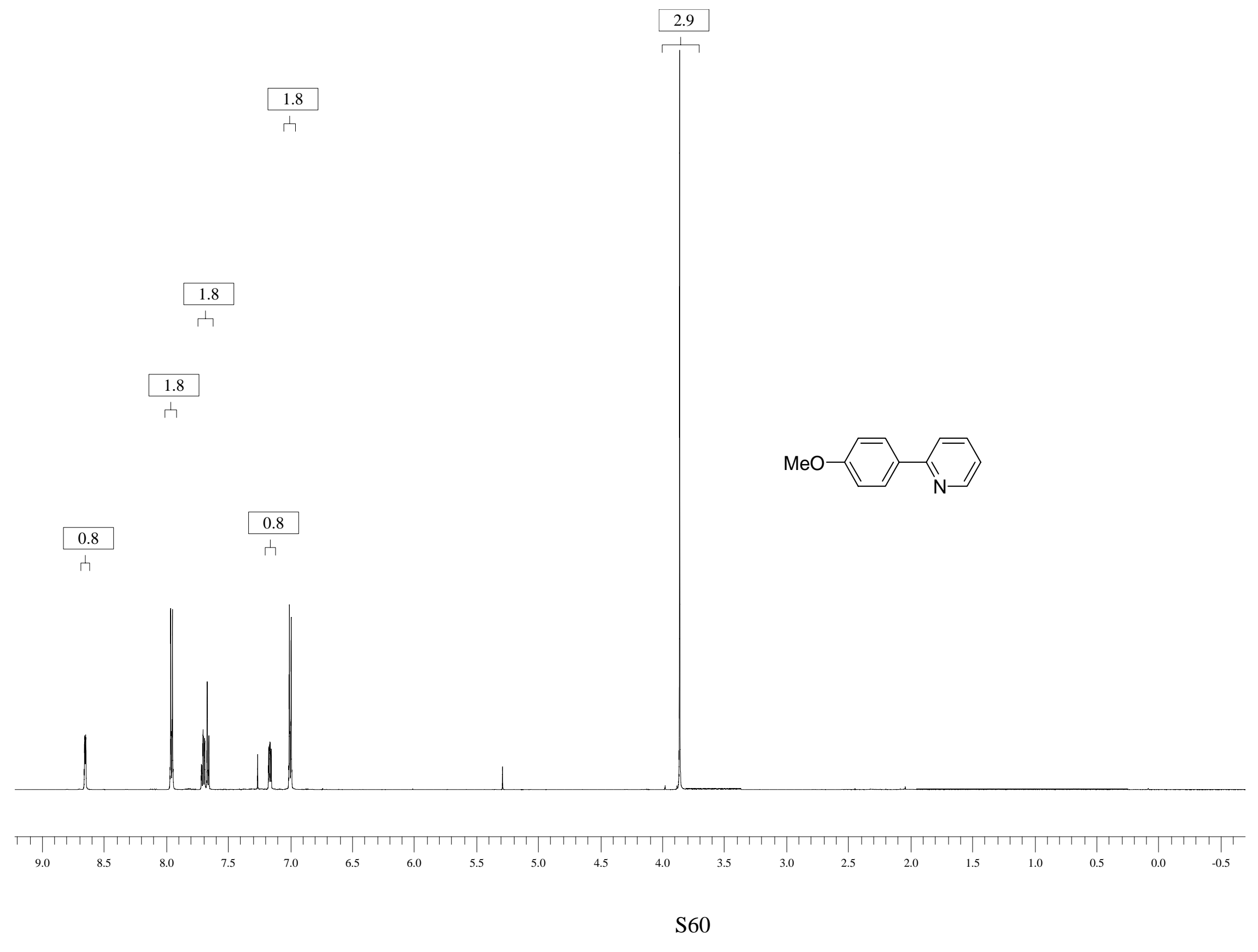




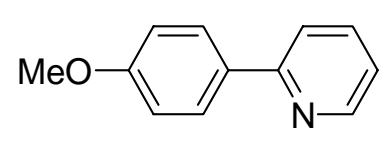

Intw

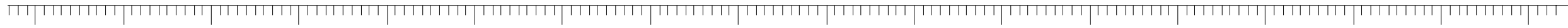




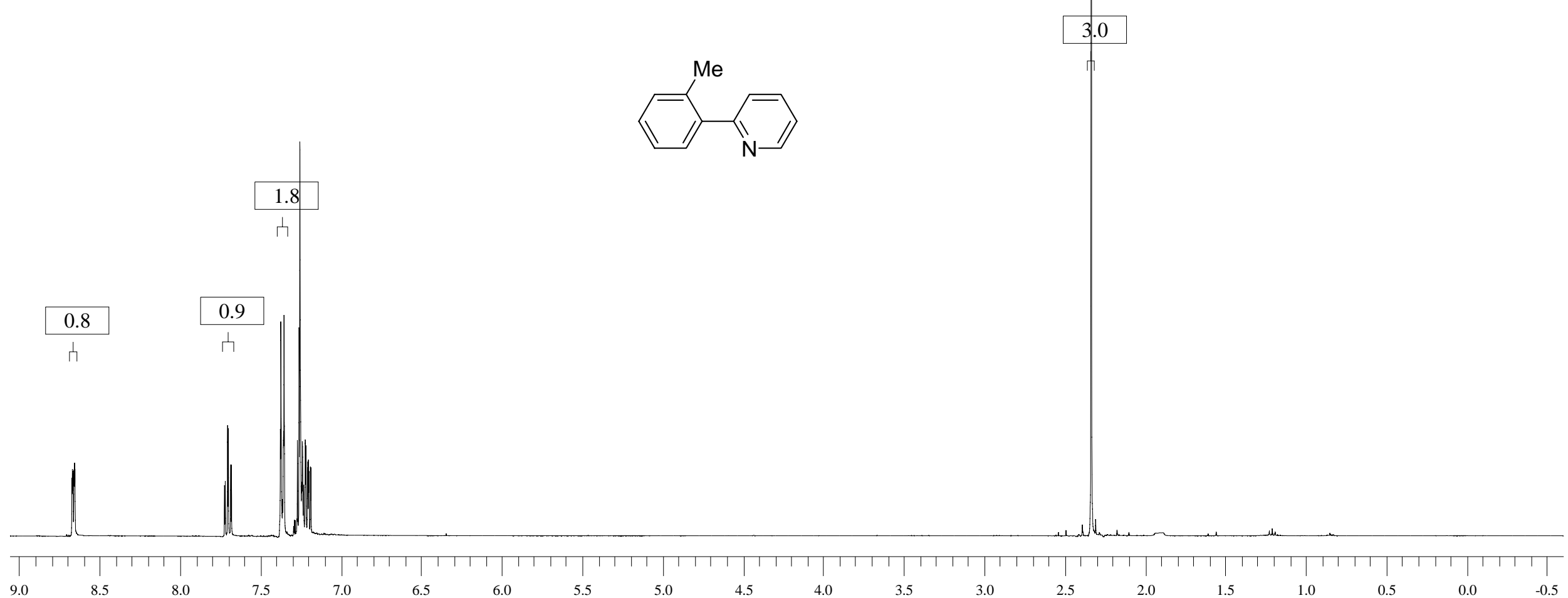




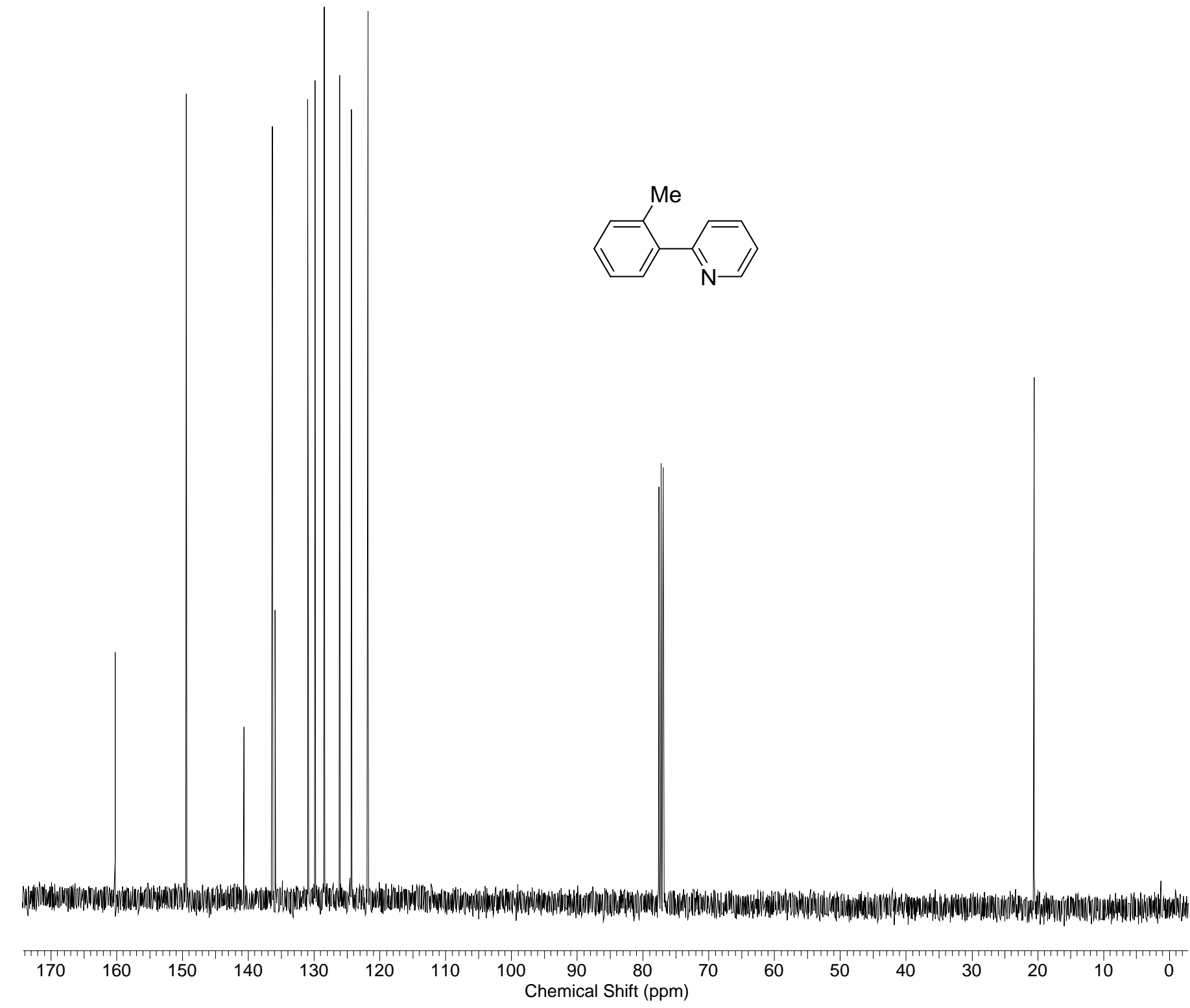




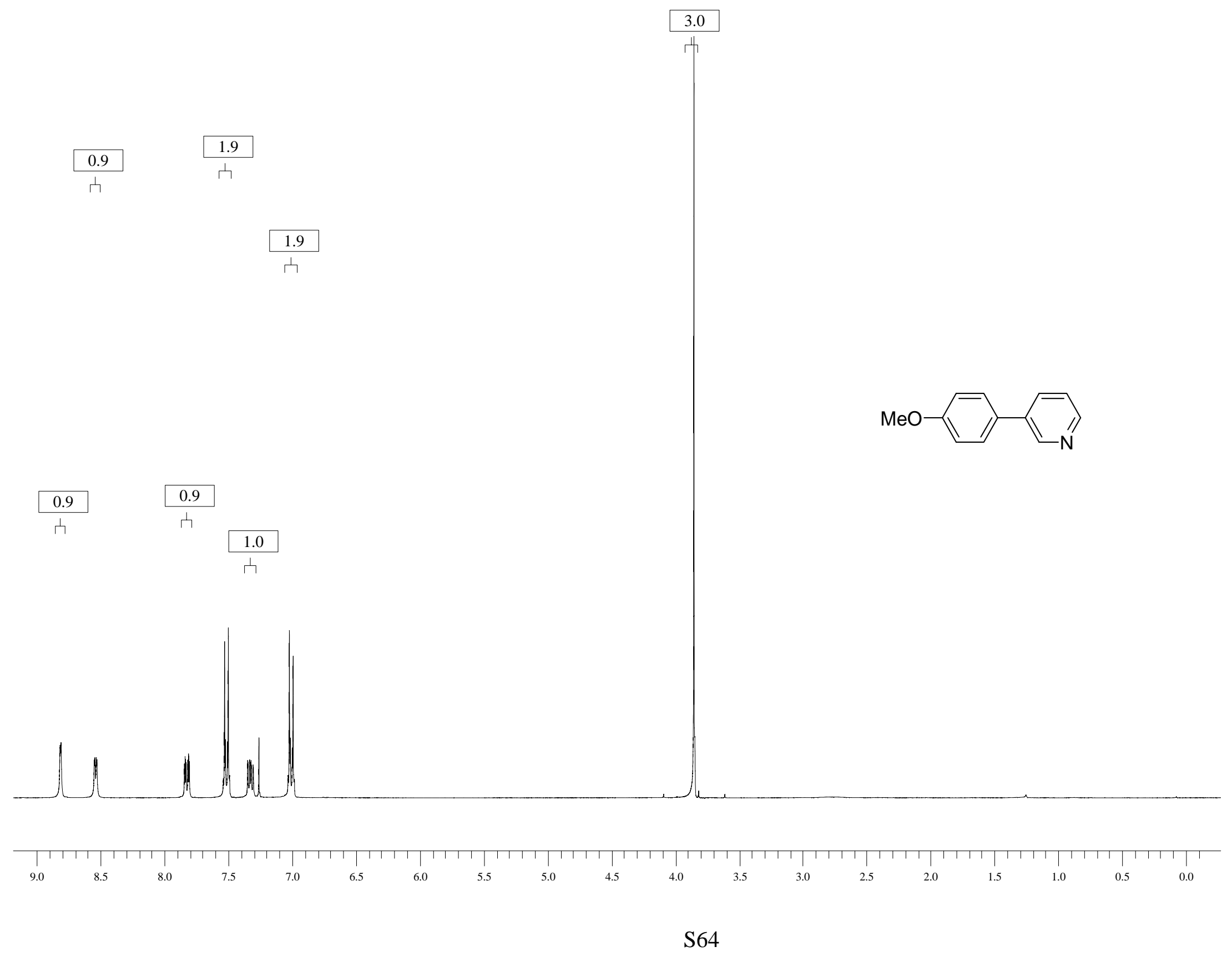




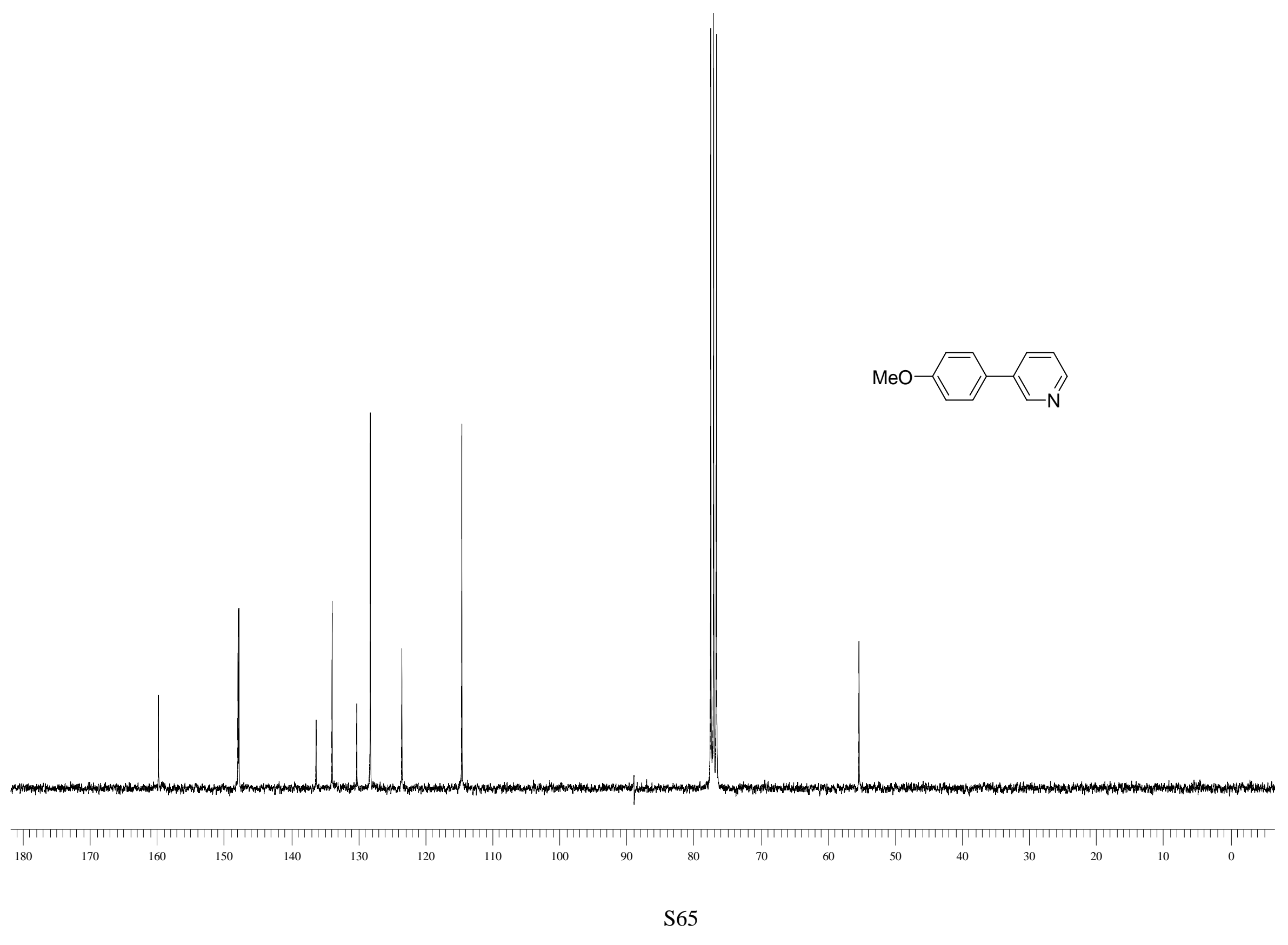




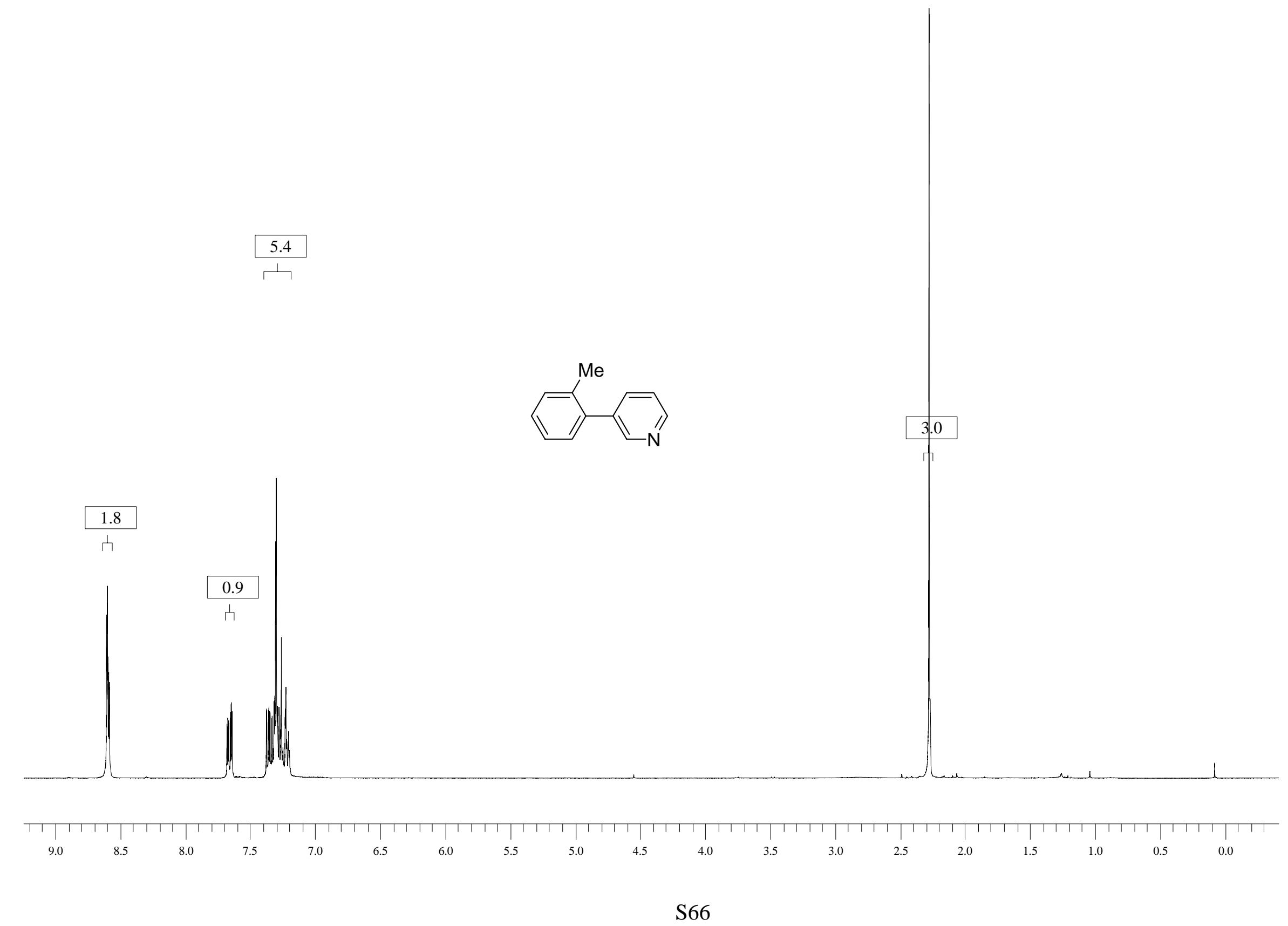




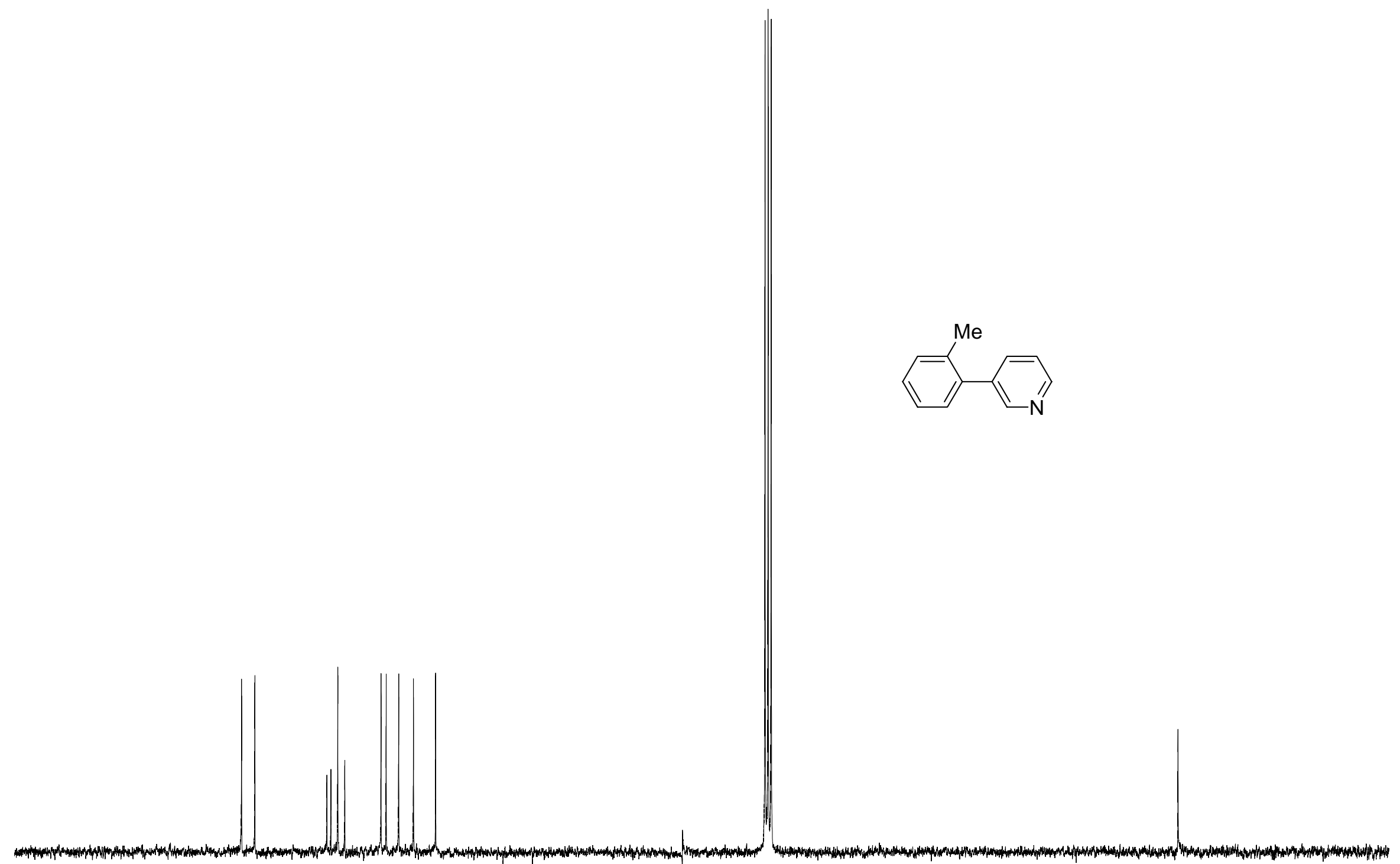




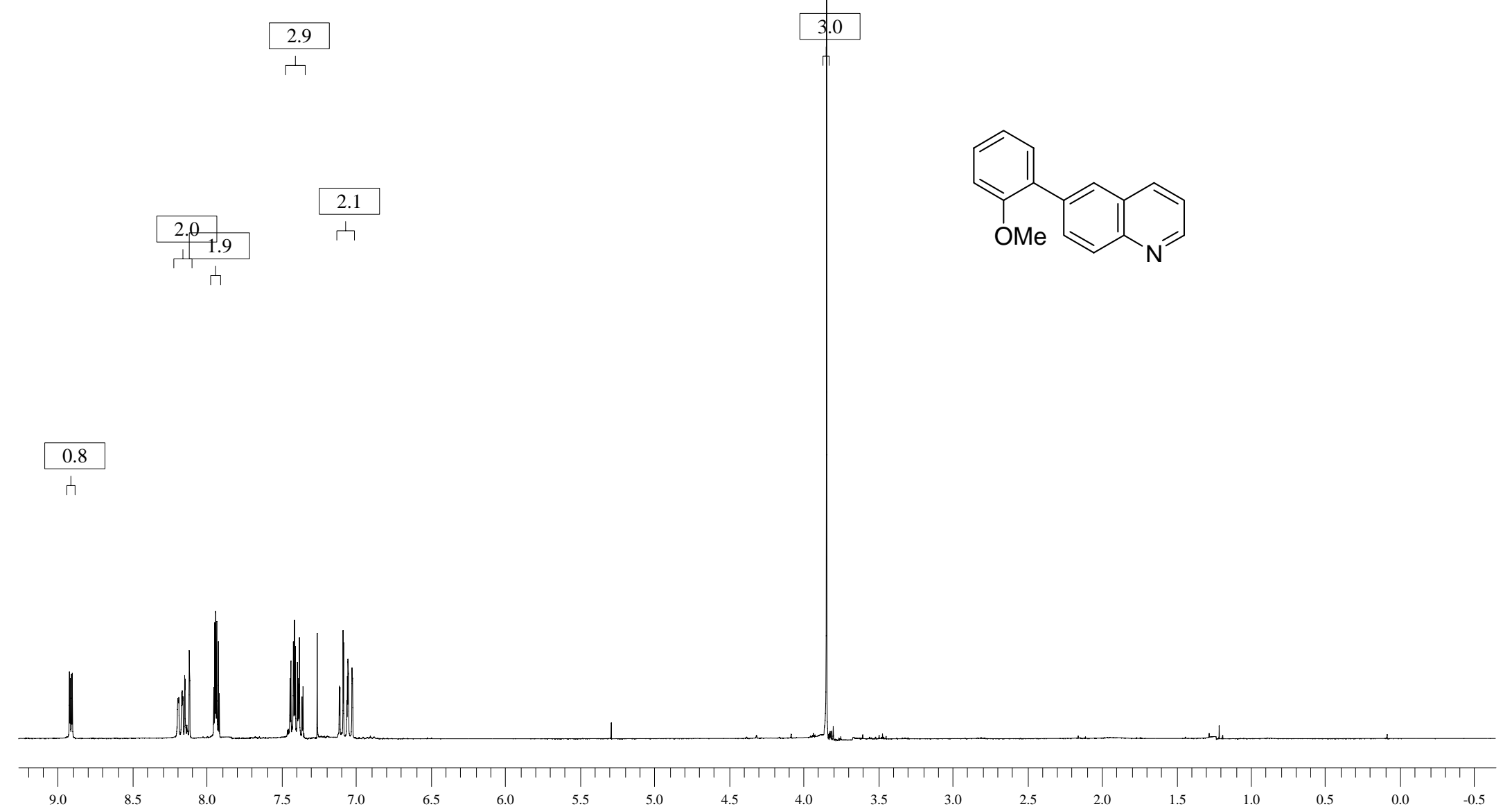




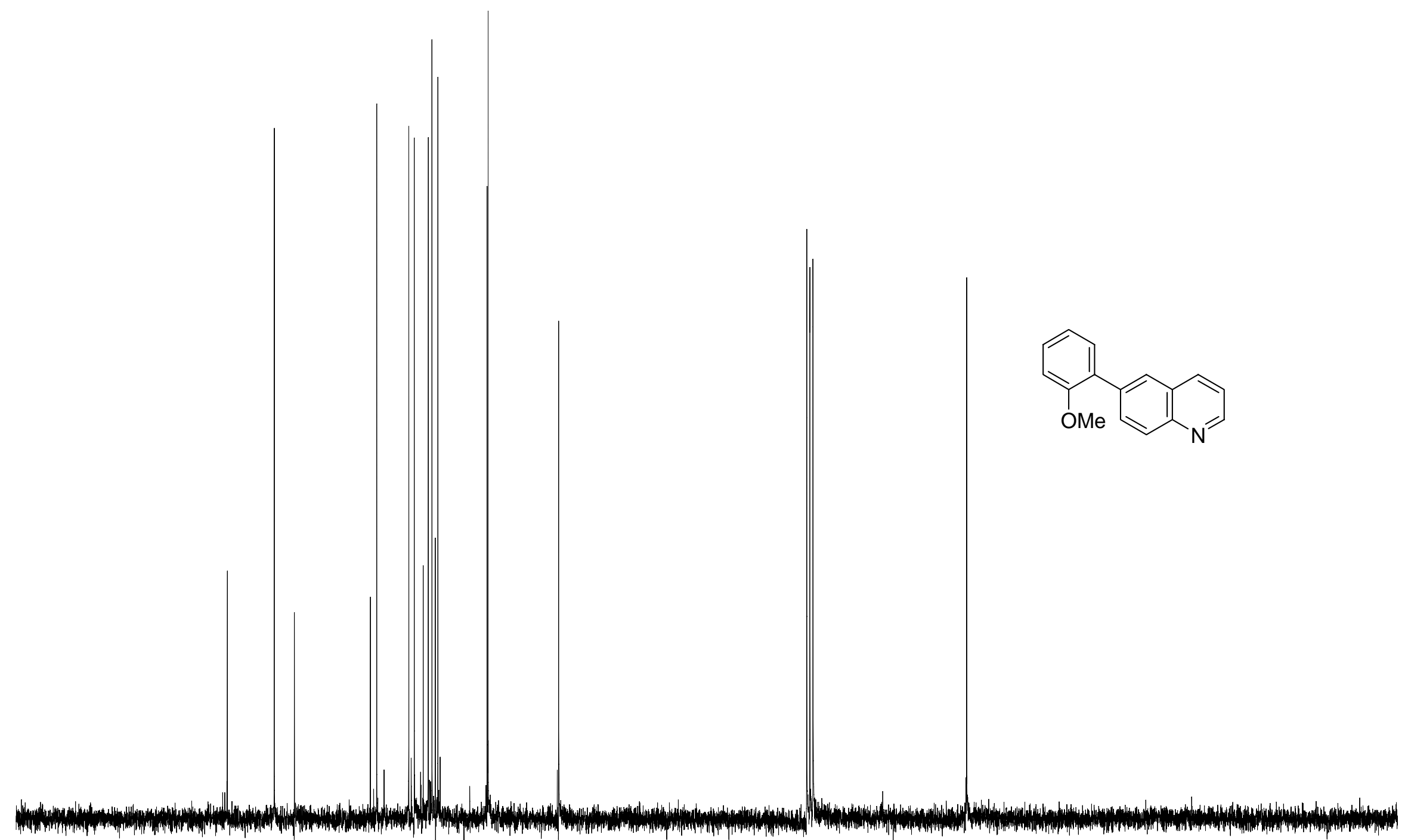

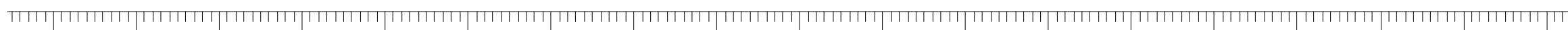
180
$170 \quad 160$
$150 \quad 140$
$130 \quad 120$

20 
\begin{tabular}{|l|l|}
\hline 1.9 & 2.0 \\
\hline$\sqcap$ & $\sqcap$ \\
\hline$\sqcap$
\end{tabular}

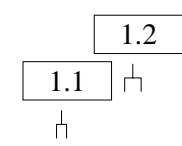

\begin{tabular}{|l|}
\hline 1.1 \\
\hline
\end{tabular}
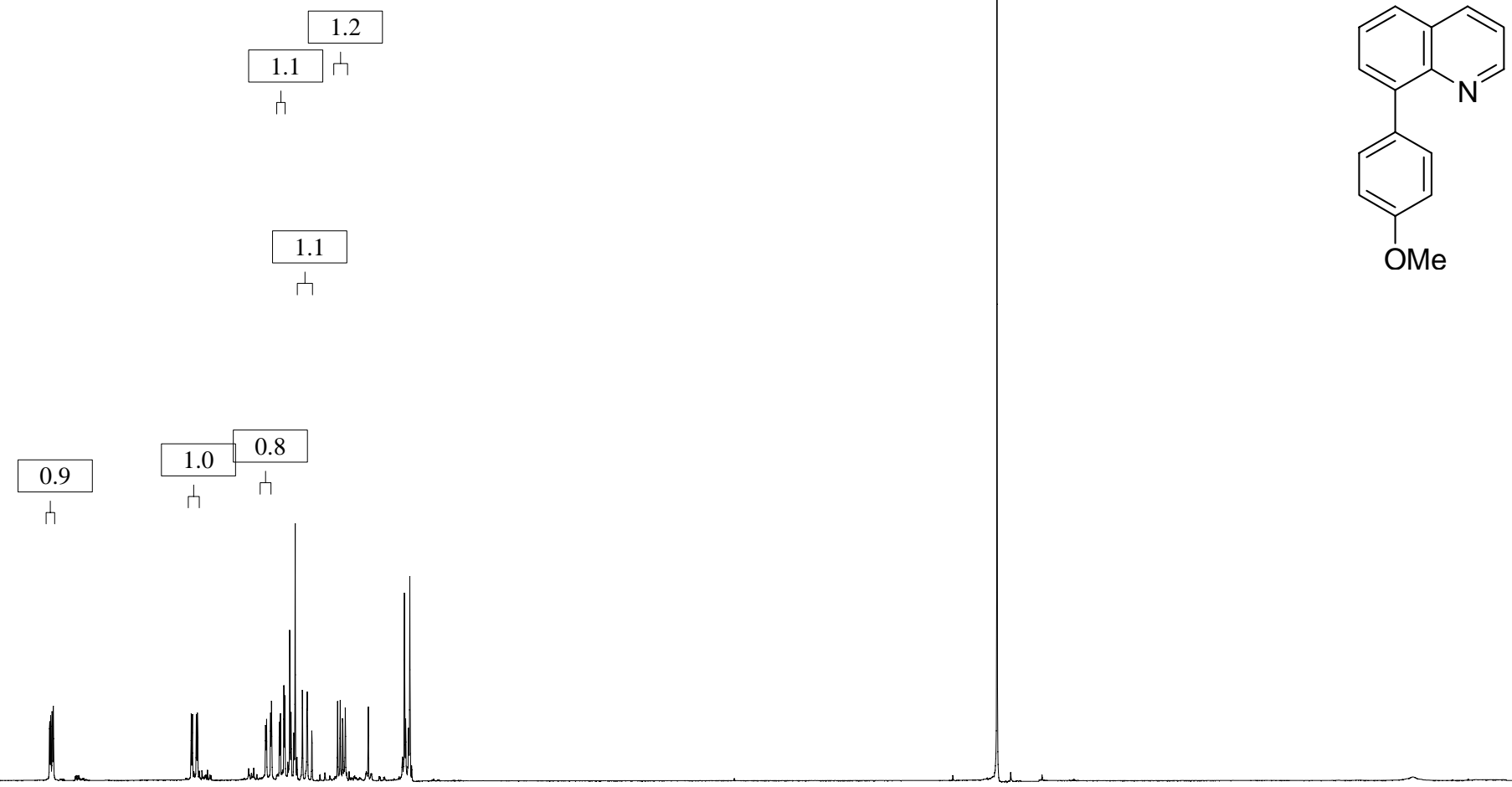

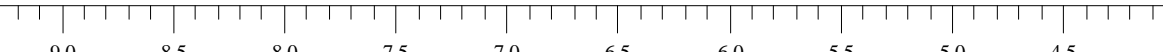

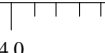

$3.5 \quad 3.0$ 


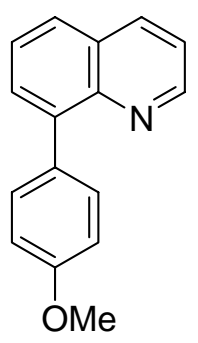

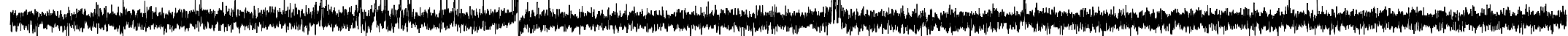

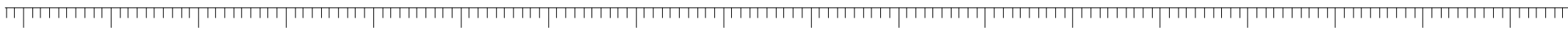

